\title{
Design of the Line Driver \\ for ADSL Communication Systems
}

by

Sergey Taryshkin

A thesis submitted to the Faculty of Graduate and Postdoctoral

Affairs in partial fulfillment of the requirements

for the degree of

\section{Master of Applied Science}

\author{
in \\ Electrical and Computer Engineering
}

Ottawa-Carleton Institute for Electrical and Computer Engineering

Carleton University

Ottawa, Ontario

(C) 2011

Sergey Taryshkin 
Library and Archives

Canada

Published Heritage

Branch

395 Wellington Street

Ottawa ON K1A ON4

Canada
Bibliothèque et

Archives Canada

Direction du

Patrimoine de l'édition

395 , rue Wellington

Ottawa ON K1A ON4

Canada
Your file Votre référence

ISBN: 978-0-494-87827-9

Our file Notre référence

ISBN: 978-0-494-87827-9

\section{NOTICE:}

The author has granted a nonexclusive license allowing Library and Archives Canada to reproduce, publish, archive, preserve, conserve, communicate to the public by telecommunication or on the Internet, loan, distrbute and sell theses worldwide, for commercial or noncommercial purposes, in microform, paper, electronic and/or any other formats.

The author retains copyright ownership and moral rights in this thesis. Neither the thesis nor substantial extracts from it may be printed or otherwise reproduced without the author's permission.
AVIS:

L'auteur a accordé une licence non exclusive permettant à la Bibliothèque et Archives Canada de reproduire, publier, archiver, sauvegarder, conserver, transmettre au public par télécommunication ou par l'Internet, prêter, distribuer et vendre des thèses partout dans le monde, à des fins commerciales ou autres, sur support microforme, papier, électronique et/ou autres formats.

L'auteur conserve la propriété du droit d'auteur et des droits moraux qui protege cette thèse. $\mathrm{Ni}$ la thèse ni des extraits substantiels de celle-ci ne doivent être imprimés ou autrement reproduits sans son autorisation.
In compliance with the Canadian Privacy Act some supporting forms may have been removed from this thesis.

While these forms may be included in the document page count, their removal does not represent any loss of content from the thesis.
Conformément à la loi canadienne sur la protection de la vie privée, quelques formulaires secondaires ont été enlevés de cette thèse.

Bien que ces formulaires aient inclus dans la pagination, il n'y aura aucun contenu manquant. 
The information provided in this thesis is part of the research program conducted by Professor T. Kwasniewski and his associates.

All research results provided in this thesis including tables, graphs and figures but excluding the narrative part of the thesis can be used by Prof. T. Kwasniewski and his associates for educational and research purposes including open publications. The matter of intellectual property may be pursued collaboratively by Carleton University and Prof. T. Kwasniewski as appropriate. 


\begin{abstract}
System solution for ADSL line driver is presented. The proposed solution includes high-resolution $2^{\text {nd }}$-order $\Delta \Sigma$ modulator as data encoder and high-efficiency switching power amplifier. The modulator has two-state analog output signal that allows easy integrating it with the amplifier.

The Verilog code of the $\Delta \Sigma$ modulator is written, integrated in Cadence Virtuoso Automated Design Environment and simulated. The signals delays within the modulator are evaluated. They are found to be 10 times less compared to the clock signal period provided that $0.18 \mu \mathrm{m} \mathrm{CMOS} \mathrm{is} \mathrm{used} \mathrm{for} \mathrm{the} \mathrm{modulator} \mathrm{fabrication.}$

Full H-bridge architecture is selected for the switching power amplifier. The dimensions of output switching NMOS/PMOS transistors in $0.18 \mu \mathrm{m}$ CMOS are determined for the output signal power $20 \mathrm{dBm}$. The simulation showed that the power efficiency of the amplifier is close to theoretical $50 \%$ level. The matching circuitry is suggested to be implemented off-chip. This allows reducing on-chip power dissipation.

The proposed line driver is simulated thoroughly in Cadence Virtuoso ADE. It is found that the dynamic range of the driver is $83 \mathrm{~dB}$ that corresponds to the data resolution 13.5 bits. Missing Tone Power Ratio of the driver is more than $65 \mathrm{~dB}$. The frequency response of the line driver is flat within entire ADSL band. Demonstrated performance of the proposed line driver meets all relevant ADSL specifications.
\end{abstract}




\section{Table of Contents}

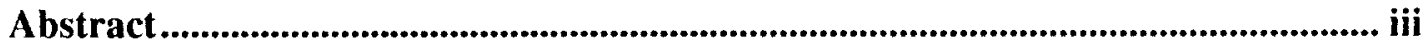

Table of Contents ..............................................................................................................iv

List of Figures.......................................................................................................................... viii

List of Tables ........................................................................................................................

Abbreviations ..................................................................................................................... xiii

1 Introduction

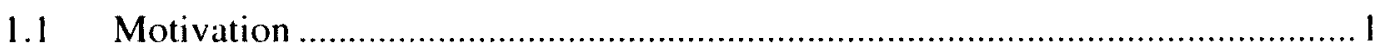

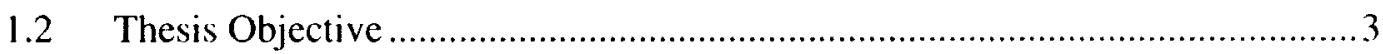

1.3 Thesis Outline

2 ADSL Signal Specifications............................................................................................

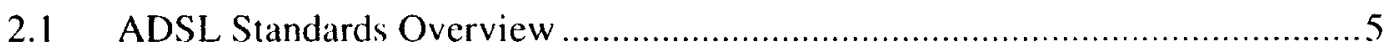

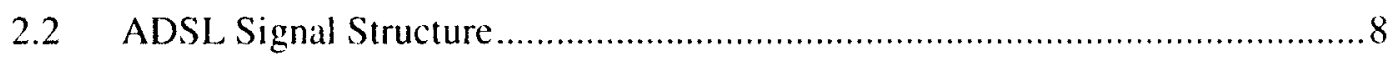

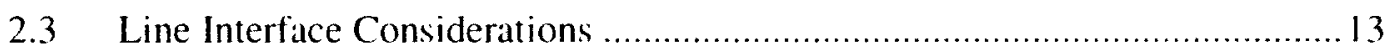

3 Discussion of $\Delta \Sigma$ Modulator for ADSL Line Driver...........................................15

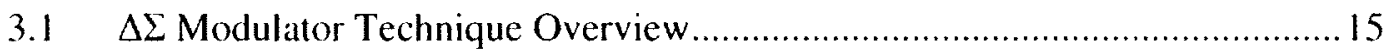

3.I.I Sampling and Quantization ................................................................ 17 


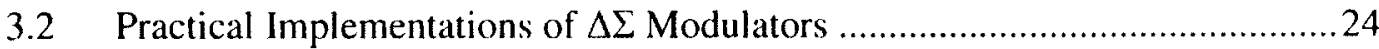

3.3 Selection of $\Delta \Sigma$ modulator Architecture for ADSL SLD .............................28

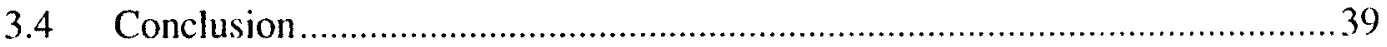

4 Switching Power Amplifier as a Potential ADSL Line Driver .............................40

4.1 The Discussion of Class D Power Amplifier .................................................. 41

4.2 Efficiency of Switching Power Amplifiers with Matching Circuit .................4. 43

4.3 Efficiency of Switching Power Amplifiers with Low-pass Filter ....................47

4.4 Self-oscillating Power Amplifiers for ADSL Applications ..........................49

4.5 Implementations of Switching Power Devices in Low-voltage CMOS...........52

4.6 Switching Output Buffer with Wideband Transformer..................................55

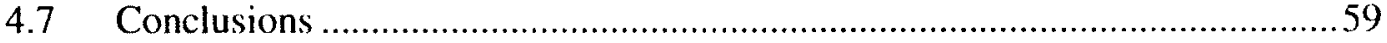

$5 \quad$ ADSL Switching Line Driver with $\Delta \Sigma$ Modulator..............................................61

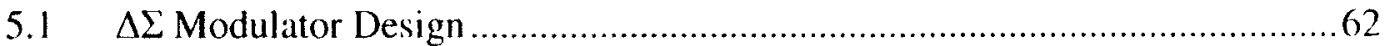

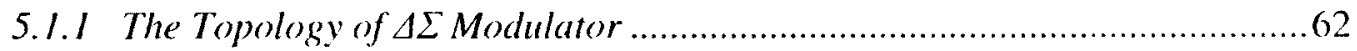

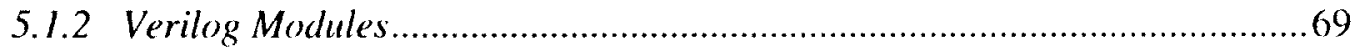

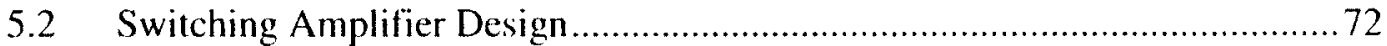

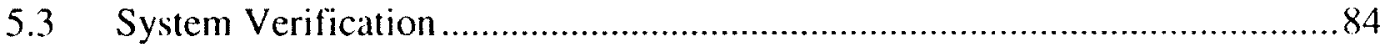

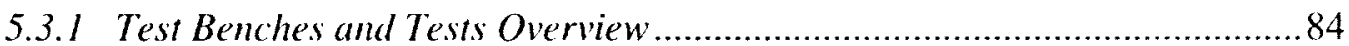


6 Conclusions and Future Work

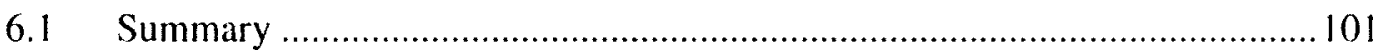

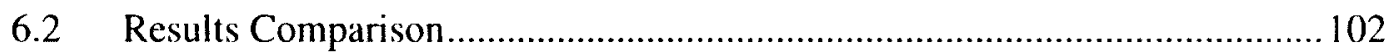

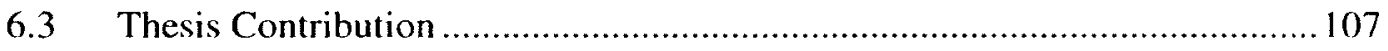

6.4 Recommendations and Future Work ......................................................... 109

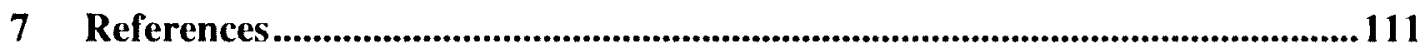

Appendix A - Matlab Processing Toolbox ...........................................................117

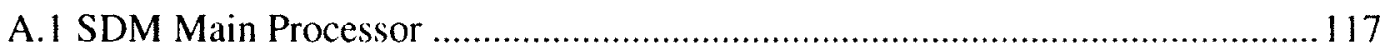

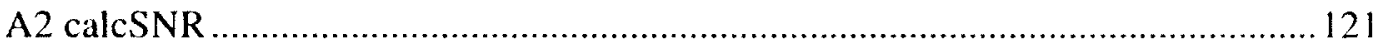

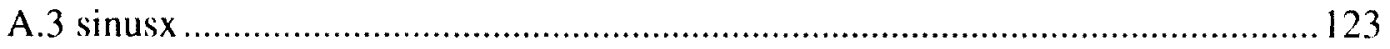

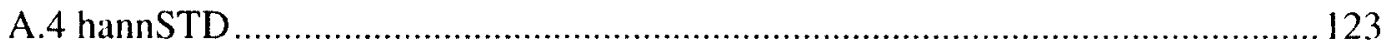

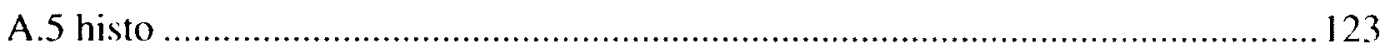

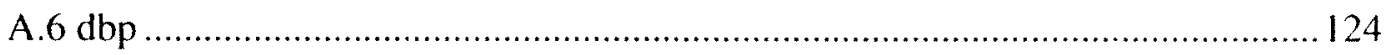

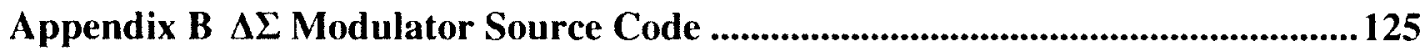

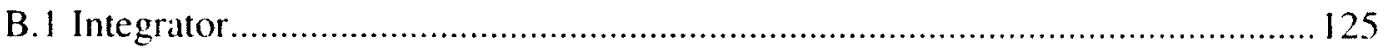

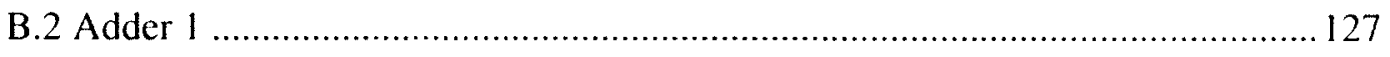

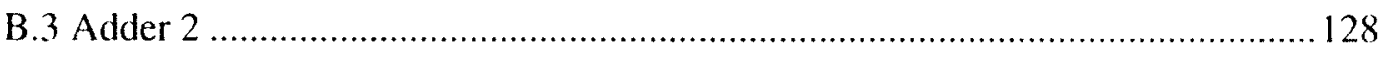




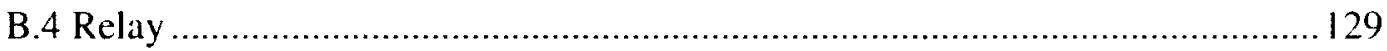

Appendix C VerilogA Source Code................................................................................131

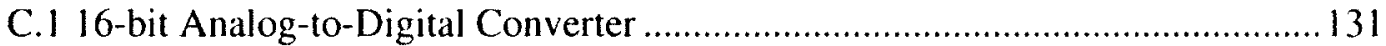

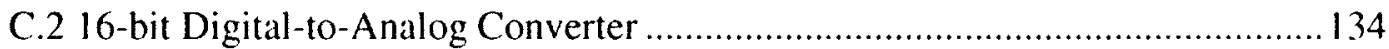




\section{List of Figures}

Figure 2.1 ADSL Spectrum Allocation, Not-overlapped Spectrum Operations........ 7

Figure 2.2 Data Allocation in Noisy Lines ......................................................

Figure 2.3 Frequency Representation of OFDM Signal ................................ 10

Figure 2.4 ADSL Transmitter, OFDM Modulator............................................... 11

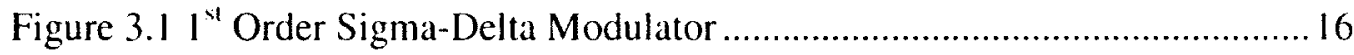

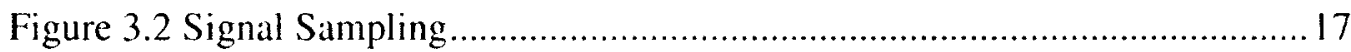

Figure 3.3 Probability Distribution of Quantization Error ................................. 18

Figure 3.4 Power Spectrum Density of Quantization Error .............................. 18

Figure 3.5 Power Spectral Densities of Quantization Errors ................................20

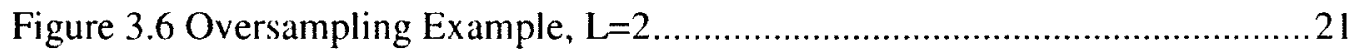

Figure 3.7 N-Order $\Delta \Sigma$ Modulator Functional Block Diagram ............................22

Figure 3.8 Signal and Noise Transfer Functions of Low-Pass $\Delta \Sigma$ Modulators .......23

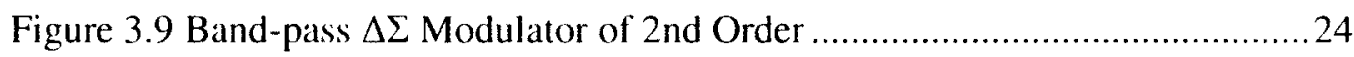

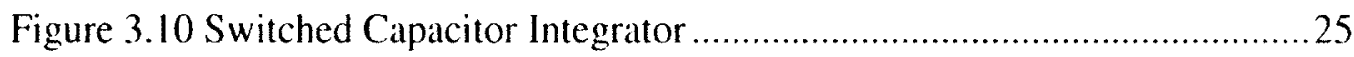

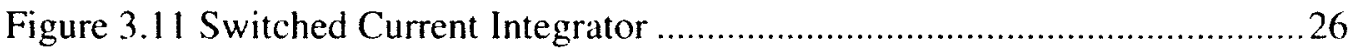

Figure 3.12 Missing Tone Power Ratio |24|_................................................29

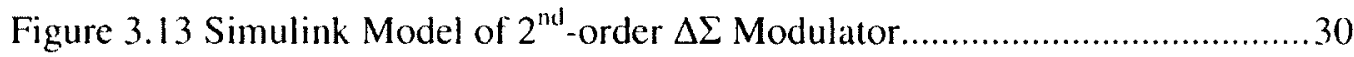

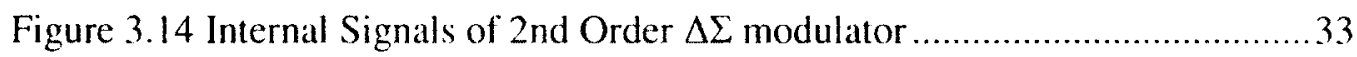


Figure 3.15 Signal Spectrum of $2^{\text {nd }}$-order $\Delta \Sigma$ Modulator's Simulink Model,

$f_{\mathrm{IN}}=250 \mathrm{kHz}$

Figure 3.16 Signal Spectrum of $2^{\text {nd }}$-order $\Delta \Sigma$ Modulator's Simulink Model,

$\mathrm{f}_{\mathrm{IN}}=250 \mathrm{kHz}$

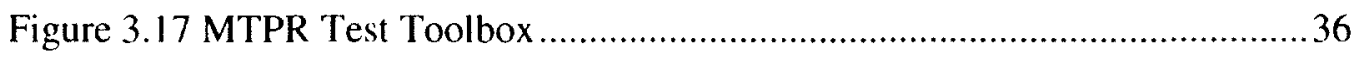

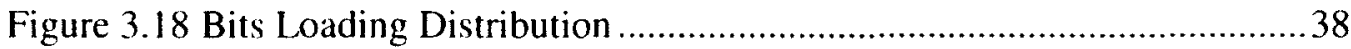

Figure 3.19 Signal Spectra at the Input and Output of 2 nd-Order $\Delta \Sigma$ Modulator ... 38

Figure 4.1 Class D Power Amplifier

Figure 4.2 Power Transfer Efficiency

.44

Figure 4.3 Equivalent Electric Diagram of Switching Amplifier

Figure 4.4 Switching Amplifier with Matching Resistor.

Figure 4.5 Switching Amplifier with Low-pass Filter. 48

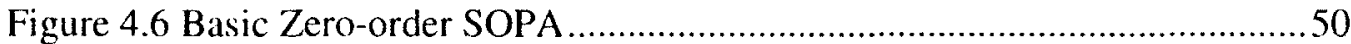

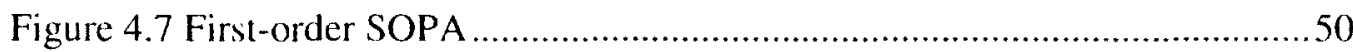

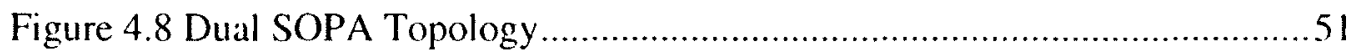

Figure 4.9 Block Schematic of High-voltage Driver .........................................53

Figure 4.10 Output CMOS Buffer with Impedance Transformer and LPF ...........56

Figure 4.11 Full H-bridge Switching Buffer..................................................57

Figure 5.1 2nd Order Sigma-Delta Modulator .....................................................63 
Figure 5.2 Functional Block Diagram of Ideal Integrator

Figure 5.3 Saturation Schematics

Figure 5.4 Signal Code Space .70

Figure 5.5 $\Delta \Sigma$ Modulator Schematic .71

Figure 5.6 Functional Block Diagram of Switching Amplifier with Connected Load

Figure 5.7 Half H-bridge Switching Amplifier Schematic .75

Figure 5.8 Half H-bridge Amplifier Pulses Shape .78

Figure 5.9 Full H-bridge Switching Amplifier .80

Figure 5.10 Full H-bridge Switching Amplifier Pulses Shape .83

Figure 5.11 Sigma-Delta Modulator Test Bench .86

Figure 5.12 $\Delta \Sigma$ Modulator's Internal Signals .87

Figure 5.13 Signals Histograms of the $\Delta \Sigma$ Modulator .88

Figure 5.14 Signal Spectrum of 2nd-order $\Delta \Sigma$ Modulator, $f_{1 N}=250 \mathrm{kHz}$,

$\mathrm{BW}=500 \mathrm{kHz}$

Figure 5.15 Signal Spectrum of 2nd-order $\Delta \Sigma$ Modulator, $\mathrm{f}_{\mathrm{IN}}=250 \mathrm{kHz}$,

$\mathrm{BW}=500 \mathrm{kHz}$ .90

Figure 5.16 DMT Signal Instantiation at the $\Delta \Sigma$ Modulator's Input. 91

Figure 5.17 ADSL Signal Processor for MTPR Evaluation .92 
Figure 5.19 ADSL Line Driver Schematic

Figure 5.20 Signal Spectrum of the Line Driver with Switching Power Amplifier,

$\mathrm{f}_{\mathrm{IN}}=250 \mathrm{kHz}, \mathrm{BW}=500 \mathrm{kHz}$

Figure 5.21 Signal Spectrum of the Line Driver with Switching Power Amplifier,

$\mathrm{f}_{\mathrm{IN}}=250 \mathrm{kHz}, \mathrm{BW}=500 \mathrm{kHz}$ .96

Figure 5.22 Dynamic Range of the Line Driver, $f_{\mathrm{IN}}=250 \mathrm{kHz}, \mathrm{BW}=500 \mathrm{kHz} \ldots .97$

Figure 5.23 Dynamic Range of the Line Driver, $f_{I_{N}}=250 \mathrm{kHz}, \mathrm{BW}=500 \mathrm{kHz} \ldots .98$

Figure 5.24 Frequency Range of the Line Driver, $\mathrm{BW}=1 \mathrm{MHz}$ .98

Figure 5.25 MTPR of the ADSL Line Driver. 100

Figure B.1 Integrator Symbol 126

Figure B.2 Adder I Symbol 128

Figure A.3 Adder 2 Symbol 129

Figure B.4 Relay Symbol. 130

Figure C.1 16-bit ADC Symbol 1.34

Figure C.2 16-bit DAC Symbol 135 


\section{List of Tables}

Table $1 \Delta \Sigma$ Modulator's Modules Delay.................................69

Table 2 Half H-bridge Switching Amplifier.................................. 77

Table 3 Half $\mathrm{H}$-bridge Switching Amplifier.................................. 79

Table 4 Full H-bridge Switching Amplifier.................................81

Table 5 Full H-bridge Switching Amplifier.................................8 82

Table $6 \Delta \Sigma$ Modulators................................................. 104

Table 7 Switching Power Amplifiers...................................... 106 


\section{Abbreviations}

ADE - Automated Design Environment

ADSL - Asymmetric Digital Subscriber Line

BW - Band Width

$\mathrm{CF}$ - Crest Factor

$\mathrm{CO}$ - Central Office

CPE - Customer Premises Equipment

CSV - Comma Separated Values

DFT - Digital Fourier Transform

DMT - Discrete Multi-Tone

DR - Dynamic Range

DSL - Digital Subscriber Line

EDA - Electronic Design Automation

FFT - Fast Fourier Transform

IFFT - Inverse Fast Fourier Transform

ITU - International Telecommunication Union

MASH - Multi-stage Noise Shaping

OFDM - Orthogonal Frequency Division Multiplexing 
OSR - Over - Sampling ratio

PA - Power Amplifier

PAPR - Peak to-Average Power Ratio

POTS - Plain Old telephone Service

PSD - Power Spectrum Density

QAM - Quadrature Amplitude Modulation

SC - Switched Capacitors

SFDR - Spurious Free Dynamic Range

SI - Switched Currents

SLD - Switching Line Driver

SNR - Signal-to-Noise ratio

SNDR - Signal-Noise Distortion Ratio

SOPA - Self-Oscillating Power Amplifier

RTL- Register Transfer Level

VDSL - Very High Speed Digital Subscriber line

UWB - Ultra Wide Band 


\section{Introduction}

\subsection{Motivation}

Modern DSL communication systems require signal processors dealing with high data rates combined with data conversion resolution of $12-14$ bits. This requirement goes along with the progress in silicon fabrication processes. Advanced functionalities built in the digital part of the communication system can be easily implemented with modern CMOS technologies that allow fabricating less expensive devices of smaller size.

At the same time, small physical dimensions of the transistor built with modern silicon technologies make the design of analog part of the devices difficult. In particular, the design of line drivers for copper cable gets challenging. The line drivers create a power bottleneck due to high Peak-to-Average Power Ratio (PAPR) of DSL signal $[1]$.

Class $A / B$ power amplifiers are still often used in DSL products. However, their efficiency and performance drop down significantly with low supply voltage headroom of modern CMOS devices. Class A/B line drivers might be replaced with Class G, Class $\mathrm{H}$, Class $\mathrm{K}$ and other high-efficient power amplifiers $[1,2]$. Even though, it is very difficult to implement these solutions in low-voltage silicon processes due to high PAPR and high linearity requirements of the quadrature amplitude modulation of Discrete Multitone (DMT) signal used in most DSL systems. 
Class D Power Amplifiers (PA) are considered as very promising solution in low-voltage devices. Switching Line Drivers (SLD) based on Class D PAs are well known for their high efficiency, for example, in audio applications.

The Self-Oscillating Power Amplifier (SOPA) proposed by T. Piessens and M. Steyaert [3] can also be classified as a switching line driver. The authors claimed relatively high power efficiency of the line driver when it used for ADSL and VDSL applications.

The SLD can be built with Delta-Sigma $(\Delta \Sigma)$ modulator as a driving circuit $|1|$. One-bit data stream from the $\Delta \Sigma$ modulator is suitable to feed baseband switching power amplifier. $\Delta \Sigma$ modulators of a proper order provide quite reasonable signal dynamic range. The combination of the modulator and switching amplifier can be a power-effective solution for DSL line drivers.

$\Delta \Sigma$ modulators are widely used in DSL products. In particular, the modulators allow cost-effective solutions for A/D converters in the receiver path of DSL Analog Front End. However, the number of publications on $\Delta \Sigma$ modulators in SLD is very limited. This solution seems challenging. $\Delta \Sigma$ modulators in the Rx path of DSL systems often have multi-bit internal feedback and output to get required accuracy and dynamic range. When used as a driver for the switching power amplifier, multi-bit $\Delta \Sigma$ modulators are not convenient. The necessity to have single-bit output signal forces a designer to increase the sampling frequency to maintain required accuracy/resolution of the modulator, but the frequency headroom is always limited due to technology restrictions. The trade-off between the accuracy and the sampling frequency is 
application specific and is determined based on relevant requirements.

The switching power amplifiers are attractive as line drivers due to their high power efficiency. The other advantage of using the switching PAs is the opportunity to employ mixed CMOS technology that doesn't require fine schematic tuning.

It should be noted that the $\Delta \Sigma$ modulators of order two and higher are usually implemented as analog circuits. It is beneficial to design the modulator as pure digital device and then to build it using standard CMOS process. Together with the switching PA, this would allow getting cost-effective solution of DSL line driver.

\subsection{Thesis Objective}

This work represents the study of $\Delta \Sigma$ modulator and switching power amplifier when they are used as the DSL line driver. Accordingly, the thesis objectives can be defined as follows:

- Analysis and justification of $\Delta \Sigma$ modulator's topology suitable for DSL applications;

- Design of the $\Delta \Sigma$ modulator and its verification in Cadence Virtuoso ADE;

- Design of switching power amplifier of $20 \mathrm{dBm}$ peak power and its verification in Cadence Virtuoso ADE;

- Characterization of the line driver composed of the $\Delta \Sigma$ modulator and the switching power amplifier against the relevant DSL specifications. 


\subsection{Thesis Outline}

The thesis is organized in accordance with the objectives defined above.

Section 2 provides the overview of DSL specifications that are relevant to the subject of the thesis. The signal structure and the modulation schemes used in the DSL systems are discussed. Details of the electrical interface specific to the DSL lines are studied.

Section 3 is devoted to the design of the $\Delta \Sigma$ modulators. The section begins with the discussion of the modulator's topologies and their performance. The overview of the modulator's implementations is given accompanied by the relevant technical publications. The architecture of the $\Delta \Sigma$ modulator proposed in this thesis work is justified.

The design of the switching power amplifier is discussed in Section 4. The signal features and the details of the line interface that might affect the efficiency of the switching PAs are studied.

The detailed design of the switching line driver proposed in this work is provided and then characterized in Section 5. The performance of the line driver is evaluated based on the simulations in Cadence Virtuoso ADE. The simulation results are processed using Matlab and Simulink.

The final section provides the summary of the work.

The Matlab scripts used in this work and Verilog code of the $\Delta \Sigma$ modulator are given in the Appendixes. 


\section{ADSL Signal Specifications}

DSL technology takes advantages of comprehensive digital signal processing (DSP) algorithms and data coding schemes. DSL communication systems have built-in intelligence to accommodate the wide varieties of data transmission signal conditions encountered with each connection through the telephone switching network. Sophisticated ASIC devices have been developed for DSL lines to implement real-time processing algorithms for these communication links $|4,5,6|$.

At the same time, as in case with almost any system, DSL ones still require analog electronics to put the signal into the phone line and to pick up small signals received at the other end. These analog subsystems should be designed properly to address specific requirements of DSL communication links.

There are several DSL technologies in a market, such as HDSL, VDSL, ADSL, to name the most generic ones. The major difference between the technologies, as they affect the line driver, is the signal structure and the amount of power put in the line by the line driver. The further discussion and study will be focusing on ADSL standard.

\subsection{ADSL Standards Overview}

Main ADSL specifications are contained in two documents published by International Telecommunication Union (ITU). The first document is G.992.1 $17 \mid$ for the systems being referred to as full-rate ADSL or G.DMT, and the second one is 
G.992.2 [8], a lower data rate solution often called G.Lite. Both systems use the DMT technique to encode transmitted data.

In full-rate ADSL systems a frequency band $\mathrm{BW}=1.1 \mathrm{MHz}$ is split for up to 256 separate tones (also called sub-carriers or sub-channels) each spaced $4.3125 \mathrm{KHz}$ apart. With each tone carrying separate data, the technique operates as if 256 separate modems were running in parallel. To further increase the data transmission rate, each individual tone is quadrature-amplitude modulated (QAM). The data to be transmitted is used to create a unique amplitude- and phase-shift combination for each carrier tone by using $I$ and $Q$ components of the signal. This combination defines so-called data symbol, which is updated at $4.3125 \mathrm{KHz}$ rate. Full rate ADSL uses up to 15 bits of data to create each symbol. This results in a theoretical maximum of $60 \mathrm{~Kb} / \mathrm{s}$ for each tone. If all 256 tones are used in parallel, the total data rate can be as high as $15.36 \mathrm{Mb} / \mathrm{s}$.

In G.Lite ADSL system the frequency band is limited to $\mathrm{BW}=0.55 \mathrm{MHz}$, accordingly, the maximum number of sub-carriers is reduced to 128 , and eight bits per sub-carrier are used only. This brings the theoretical maximum data rate down to 4.096 $\mathrm{Mb} / \mathrm{s}$.

In ADSL applications, the tones are allocated depending on the direction of communication, as shown in Figure 2.1. Most of the tones are used for data transfer from the Central Office ( $\mathrm{CO}$ ) to an end-user modem often referred to as Customer Premises Equipment (CPE). This direction of communication is called "downstream". The direction of communication from the CPE to the CO is called "upstream". The assignment of more tones for the downstream direction makes sense from an Internet- 
access point of view as most users download more information than they upload. Most upstream communication with a server is simply to request information to be sent quickly downstream. This difference in data rates up- and downstream is the reason that ADSL is called asymmetric DSL.

Maximum Power Spectral Density (PSD) of all tones is specified in G.922.1 and G.992.2 standards as shown in Figure 2.1 below. The PSD specifications determine the amount of signal power that has to be put on the phone line. The power levels are restricted to minimize crosstalk and interference into other phone lines contained in wire bundle en route to and from the central office.

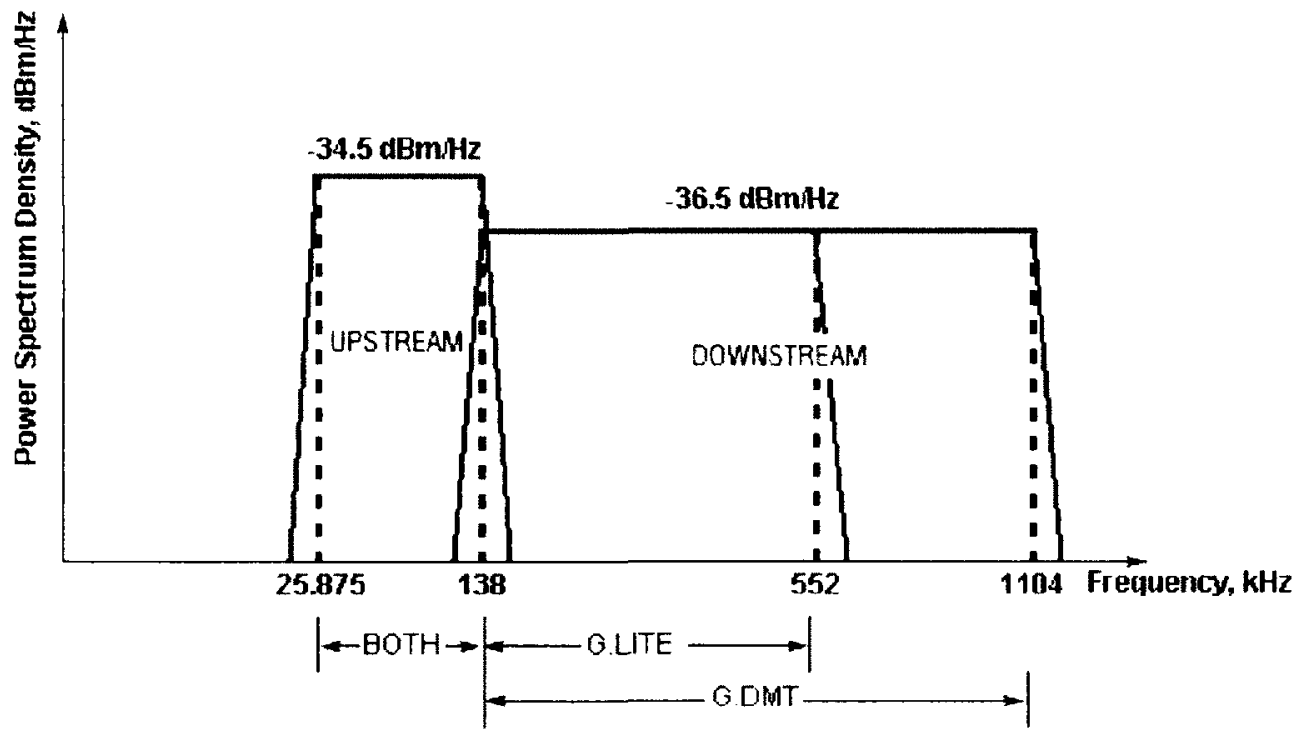

Figure 2.1 ADSL Spectrum Allocation, Not-overlapped Spectrum Operations

The downstream signal power is much higher than the upstream one because of wider bandwidth used for the transmission. For the same reason, full-rate G.DMT ADSL carries more line power than G.Lite for downstream transmissions. Upstream power is the same for both full-rate and G.Lite DSL implementations. 
ADSL line driver should be able to generate the signal with power $13 \mathrm{dBm}$ for upstream and up to $20 \mathrm{dBm}$ for downstream channels.

\subsection{ADSL Signal Structure}

As mentioned above, DMT technique splits the frequency bandwidth into a set of smaller sub-channels. The data is encoded in each sub-channel using QuadratureAmplitude Modulation (QAM). The QAM order in each sub-channel is defined by Signal-Noise Ratio (SNR) that is measured dynamically for each tone.

The intelligence is built in DSL modems mostly to obtain the fastest data rate for any set of line conditions. In order to gauge the conditions on a line $\mathrm{CO}$ modem initiates so-called "training-up" session. During this session, the modems at both ends of a line transmit maximum and equal amount of data per tone/sub-channel. When "training-up" signal sequence is received at the opposite side of the line, the modems processors calculate errors and determine the signal-noise ratio in each sub-channel. The SNR distribution throughout the tones is then sent back to an originating modem. The modem"s DSP automatically allocates less data in "noisy" sub-channels using QAM of lower order. Accordingly, QAM of higher order is used in "clean" sub-channels that allow pumping more data in them. This algorithm maximizes the data throughput for a particular line. Figure 2.2 illustrates the algorithm of data allocation used in DSL systems.

Noise can contaminate any number of sub-channels to render them completely unusable, or useful, but with less than maximum possible data capability. Additionally, 
the sub-channels at higher frequencies of the bandwidth are attenuated more than at the lower ones, particularly over longer phone lines used to make a connection.
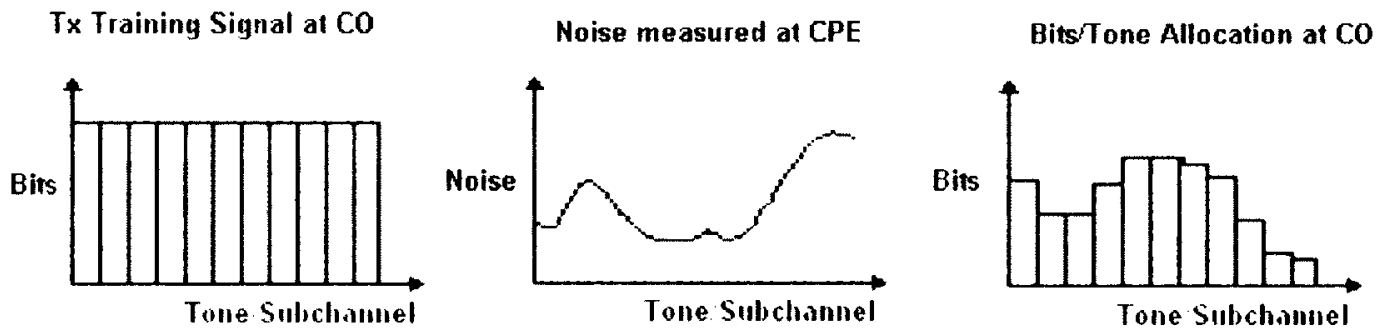

Figure 2.2 Data Allocation in Noisy Lines

Another issue that can render particular tones unusable and create transmission errors is distortions from the amplifier that drives the line. Distortion products from any Tx tones of DMT signal, such as harmonics, intermodulation, induce energy at the frequencies used by other tones. This energy also contaminates the data content of the tones and results in less sub-channel data capability or even fewer tones being used for the data transmission.

The encoding scheme of ADSL signal is classified in literature as Orthogonal Frequency Division Multiplexing (OFDM). The primary advantage of OFDM is its ability to cope with severe channel conditions (for example, attenuation of high frequencies in a long copper wire, narrowband interference and frequency-selective fading) without complex equalization filters. Channel equalization is simplified, because OFDM is treated as set of slowly-modulated narrowband signals rather than one fast-modulated wideband one. The low symbol rate in each narrowband subchannel/tone makes the use of a guard interval between symbols affordable that allows to handle time-spreading and to eliminate inter-symbol interference. 
In OFDM, the sub-channel frequencies are selected so that the narrowband signals are orthogonal to each other, meaning that cross-talk between the sub-channels is eliminated and inter-carrier guard bands are not required.

The orthogonality requires that the space $\Delta f$ between sub-channel frequencies is reverse proportional to the duration of data symbol $\mathrm{T}$ :

$$
\Delta \mathrm{f}=1 / \mathrm{T}
$$

Orthogonality increases the spectral efficiency of OFDM signals by allowing the overlapping of spectra of the sub-channel signals. The representation of 4-tone OFDM signal in frequency domain is shown in Figure 2.3 below [9]

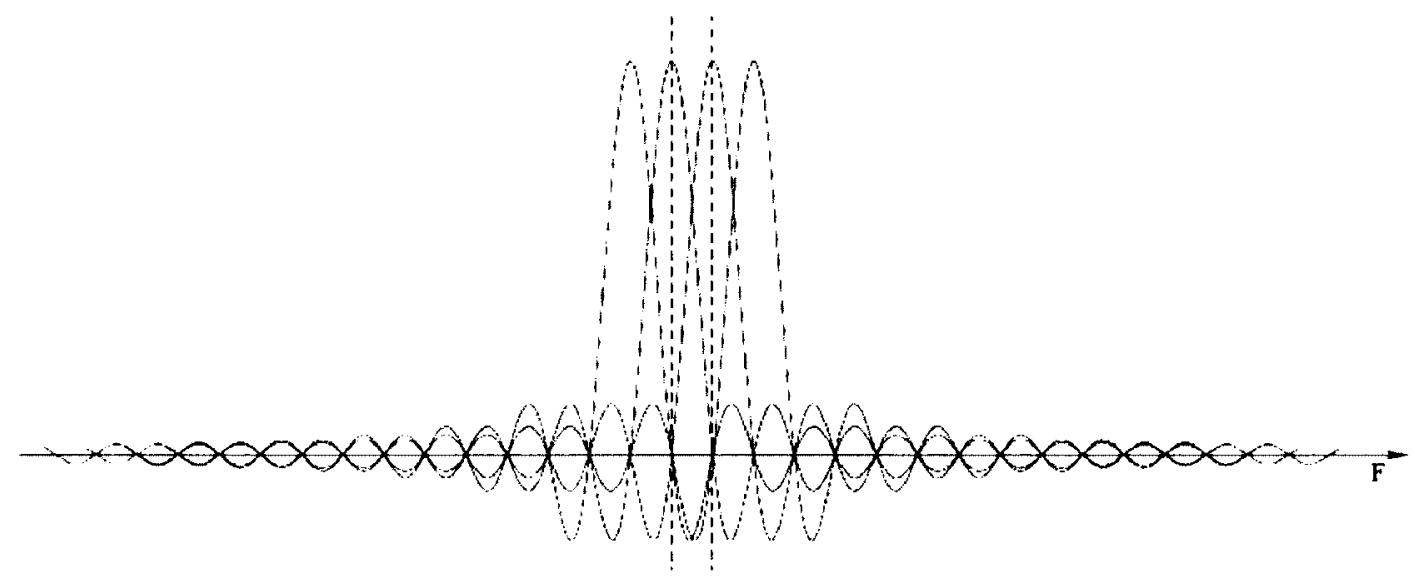

Figure 2.3 Frequency Representation of OFDM Signal

The harmonics of narrowband signals/tones around their sub-channel central frequencies overlap and partially compensate each other as their phase switches by $180^{\circ}$ from one harmonic to the next one. As a result, the noise floor due to interference between sub-channels is relatively low and grows slower with the number of subchannels compared to non-orthogonal signals. 
The orthogonality allows an easy implementation of OFDM receiver and transmitter using FFT and IFFT algorithms respectively. Simplified block diagram of Tx modulator is shown in Figure 2.4 below.

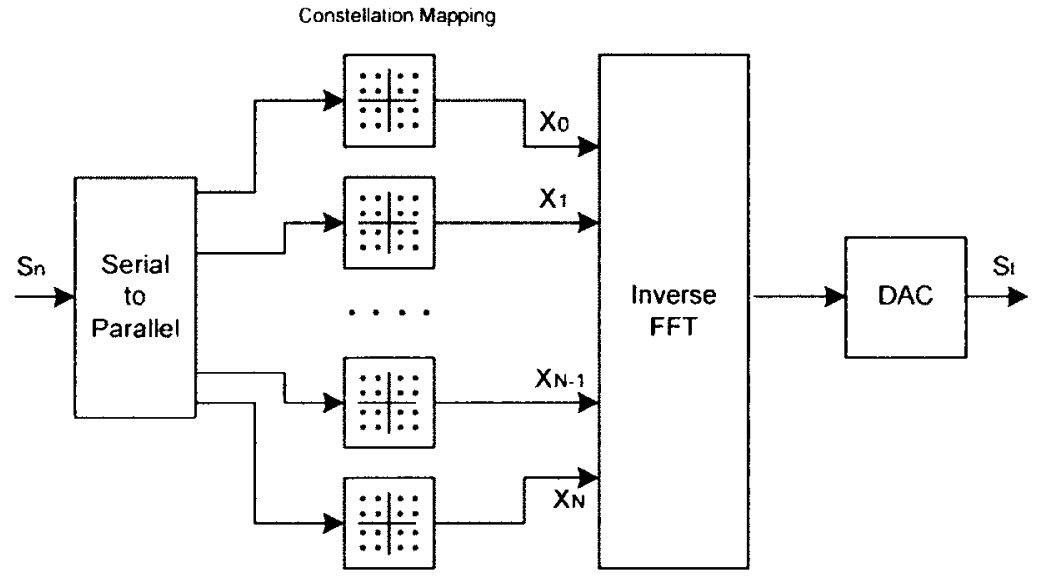

Figure 2.4 ADSL Transmitter, OFDM Modulator

$\mathrm{S}_{\mathrm{n}}$ is a serial stream of binary digits. This data stream is first de-multiplexed into $\mathrm{N}$ parallel streams, and each one mapped to a symbol stream $\mathrm{X}_{\mathrm{i}}$ using modulation constellation (QAM for ADSL). The constellations could be of different order, so some streams may carry a higher bit-rate than others.

An inverse FFT is computed on each set of symbols, producing a set of complex time-domain samples. The real and imaginary components are first converted to the analogue domain using Digital-to-Analog Converters (DAC's). The analog signals can be then up-converted to higher frequency and quadrature-mixed for further amplification in the transmitter. 
No signal up-conversion is required in DSL devices, and baseband signal from DAC output is applied to line terminals directly or through power amplifier, if necessary.

Baseband DMT signal placed on the line looks basically like white noise because many signals of changing amplitude and phase are combined simultaneously. The changes of each signal are considered random as they result from an arbitrary sequence of data bits comprising the transmitted information. The signals can stack from time to time and create a large peak signal. If this peak is not processed properly (for example, if the line-driver amplifier clips), data error can occur.

Term Peak-to-Average Power-Ratio (PAPR) is used in literature to characterize such signal behavior. This term is similar to the term of Crest Factor (CF). The PAPR determines the peak value of the voltage put on the line over a time with respect to the RMS voltage level:

$$
\mathrm{V}_{\text {peak }}=\mathrm{PAPR} * \mathrm{~V}_{\mathrm{RMS}} \quad(2-1)
$$

For OFDM signals, PAPR depends on the number of tones/sub-carriers comprising the signal. For ADSL signal with 256 tones PAPR can be as high as $12 \mathrm{~dB}$. In reality, the number of tones used during some particular communication session is less than maximum possible. Also data capability of each sub-carrier/tone is different. This usually reduces PAPR value a bit.

However, the PAPR remains high enough to challenge the design of ADSL line driver. This factor determines both the minimum supply voltage required to prevent clipping of the signal at the output of analog line driver and also the peak output power 
capability of the driver. The PAPR actually defines the dynamic range of the line driver and, hence, its efficiency, in particular, when power amplifiers of Class A and B are used.

\subsection{Line Interface Considerations}

The characteristic impedance of ADSL communication line is $100 \Omega$. This determines the current at the line input terminals if the supply voltage of the line driver is defined.

A transformer is usually used to connect the DSL transceiver to the line. The transformer is selected for a flat, distortion-free frequency response from $20 \mathrm{kHz}$ to $\mathrm{I}-2$ $\mathrm{MHz}$ to cover the full operational frequency band.

One of the functions of the transformer is to provide DC isolation between DSL modem front-end and the line. At the same time, the turn's ratio of the transformer can be used to provide a voltage gain to the signal routed to the line. The turn's ratio is defined mostly by the power supply voltage for the line-driver amplifiers. By stepping up the signal from the driver to the line via the transformer, the signal voltage swing at the amplifiers output can be reduced. As an ideal transformer has unity power gain, i.e. equal power in the primary and secondary coils, while the voltage is stepped up. the current is stepped down. The consequence of using a step-up transformer is beneficial in that lower, more convenient supply voltage can be used, but the amplifiers must have higher current driver capability.

The negative impact of step-up transformer is that it steps down the signal coming back from the phone line, so the sensitivity of DSL modem might saturate. 
Appropriate modem's front-end architectures should be employed to avoid or to minimize the sensitivity degradation of the modem with transformer line interface. 


\section{Discussion of $\Delta \Sigma$ Modulator for ADSL Line}

\section{Driver}

As shown in Figure 2.4 above the signal generated by the OFDM modulator is routed to the DAC to produce its analog representation. The DAC might be followed by an amplifier that is necessary to increase the power of the analog signal to the required level.

The manipulations with OFDM signal in analog domain are challenging. Although the design solutions of 15-16 bit DACs are known, their application for ADSL systems might be impractical due to unique set of resolution, speed and dynamic range parameters. Also, the design of linear power amplifier for the signals with high PAPR is difficult. As noted earlier, the linear amplifier appears to be a bottleneck of ADSL modem.

As an alternative design solution of ADSL transmitter path, Switching Line Driver composed of $\Delta \Sigma$ modulator followed by the switching power amplifier can be used. The $\Delta \Sigma$ modulator converts the signal from the IFFT DSP to single-bit data stream, which is then appropriately amplified to maintain required signal swing at the input terminals of the line.

\section{1 $\Delta \Sigma$ Modulator Technique Overview}

$\Delta \Sigma$ modulator presents attractive solution. It samples signals at much higher rate than the Nyquist rate. The over-sampling relaxes anti-alias requirements and actually 
trades the resolution in time for resolution in signal amplitude. Noise shaping property of the $\Delta \Sigma$ modulator allows it to achieve high resolution even when simple components such as single-bit quantizer are used.

Block diagram of $1^{\text {st }}$ order $\Delta \Sigma$ modulator is given in Figure 3.1 below. Input signal, analog or multi-bit digital, is applied to the summing/delta junction along with the one-bit output. The delta value is applied to the integrator (sigma), which feeds a comparator. The comparator might be multi- or single-bit device. In latter case, singlebit comparator can feed switching power amplifier.

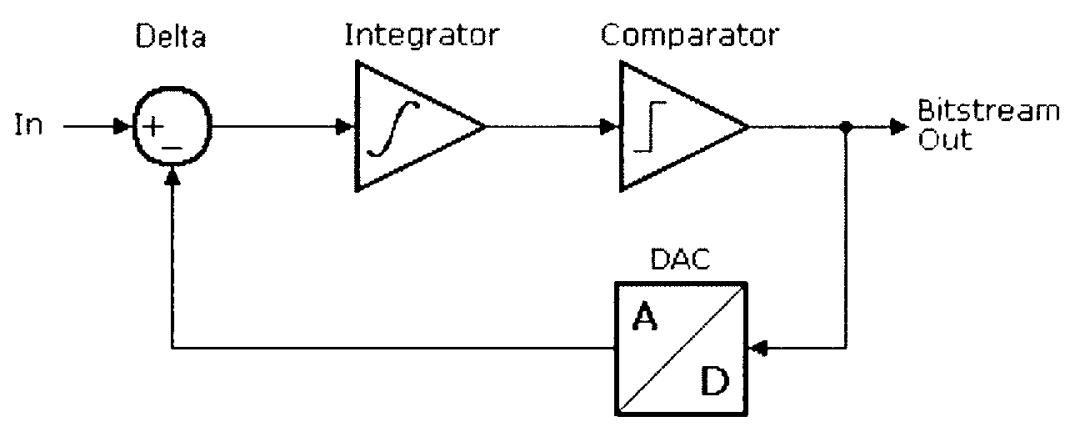

Figure $3.11^{\text {st }}$ Order Sigma-Delta Modulator

The $\Delta \Sigma$ modulator is proposed to improve the performance of well-known $\Delta$ modulator, which exhibits excessive comparator's switching and so introduces extra noise at the output. To reduce the noise due to the switching the Integrator module is supposed to be placed at the output of the Comparator module. However, due to the linearity of DAC and Delta module's operation, the Integrator module is inserted after the Delta module. This makes the design of the $\Delta \Sigma$ modulator much easier.

The $\Delta \Sigma$ modulator allows discrete as well as continuous time/signal implementations. Both options employ over-sampling technique and feature noise 
shaping to achieve high signal resolution. Discrete $\Delta \Sigma$ modulator is studied further for the sake of the application being considered.

A quick review of quantization noise theory and signal sampling theory is useful before diving deeper into the $\Delta \Sigma$ modulator details.

\subsubsection{Sampling and Quantization}

In discrete applications signals are represented by sequence of the signals samples taken at equidistant time intervals $\mathrm{T}_{\mathrm{S}}$. Each sample is approximated by a digital code of finite length, or quantized.

Shannon's sampling theorem states that the sampling frequency $f_{S}=1 / T_{S}$ should be at least twice higher that the signal bandwidth $F_{B W}$ in order to recover the sampled signal back to continuous-time without distortion. Sampling frequency $f_{S}=2 \cdot F_{B W}$ is called Nyquist frequency. Also, when signal is sampled, its spectrum is copied and mirrored at multiples of the sampling frequency as shown in Figure 3.2 below.
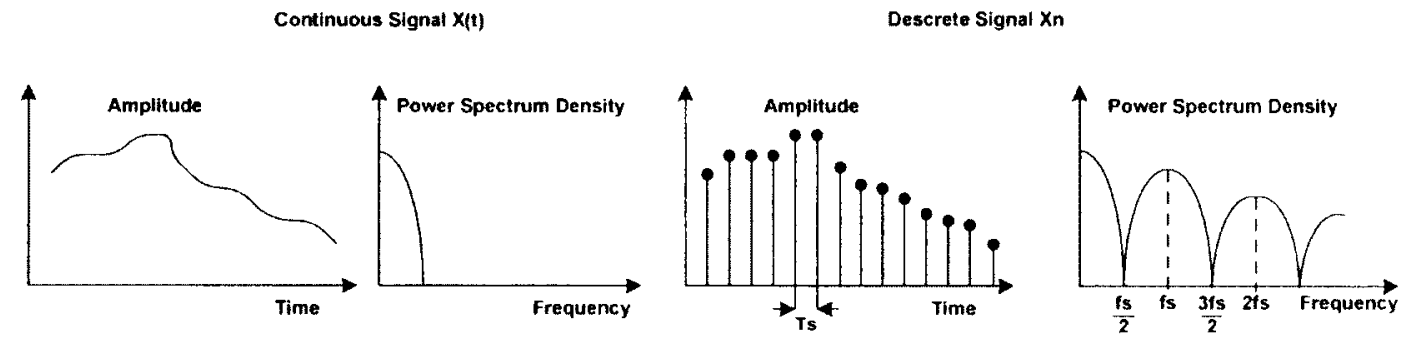

Figure 3.2 Signal Sampling

Mirrored signal spectrum components are called aliases. Since aliasing is produced above and below signal original spectrum, the signal must be low-pass filtered before being sampled. Otherwise, its high-frequency content will produce aliases in the 
baseband. Also, since high-frequency aliases are usually unwanted, they must be filtered out during signal conversion to continuous-time signal representation. Both presampling and post-sampling filtering is referred to as anti-alias filtering.

The signal sampled at some moment of time is represented by some number that corresponds to its amplitude at that time. With a B-bit code length, there are $2^{\mathrm{B}}$ different available numbers that can be used to characterize the signal amplitude. The amplitude is rounded off to the closest number. This is called signal quantization. The quantization error e[n], i.e. the difference between the actual signal amplitude and its round-off value, is considered as a white noise with zero mean value and uniform probability distribution function $\mathrm{P}(\mathrm{e})$ as shown in Figure 3.3 below.

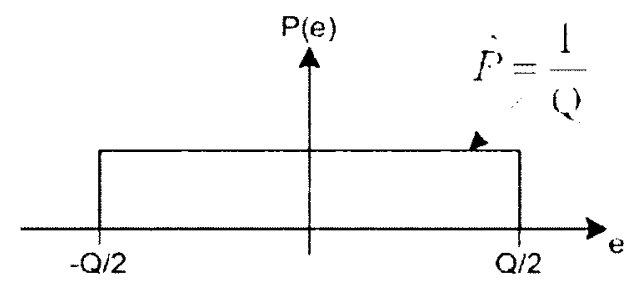

Figure 3.3 Probability Distribution of Quantization Error

In the drawing above $Q$ is a quantization step.

Since the quantization error is random, i.e. white noise, the power spectrum density of the error $S_{e}$ is also uniform within Nyquist frequency band.

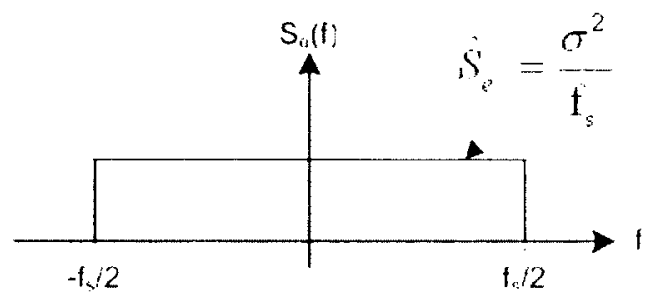

Figure 3.4 Power Spectrum Density of Quantization Error 
The variance or the power of the quantization error is calculated as follow.

$$
P_{e}=\sigma_{e}^{2}=E\left[e^{2}\right]=\int_{-Q}^{Q_{2}^{2}} e^{2} P(e) d e=\frac{1}{Q_{-Q}} \int_{-Q_{2}^{2}}^{Q^{2}} e^{2} d e=\frac{Q^{2}}{12}
$$

Signal-to-quantization-noise ratio (SQNR) is one of the most important parameters when evaluating digital systems. For sinusoidal signal of maximum-level amplitude $A=2^{13-1} \cdot Q$ the SQNR can be easily calculated and is as follow.

$$
S N Q R=10 \cdot \log \left(\frac{P_{x}}{P_{e}}\right)=10 \cdot \log \left(\frac{\frac{y^{-1-1} Q^{2}}{2}}{\frac{Q^{2}}{12}}\right)=6.02 B+1.76[\mathrm{~dB}]
$$

When signal sampling frequency is higher than Nyquist frequency $f_{S O}=L^{*} f_{S}$, the quantization error $\mathrm{e}^{\prime}[\mathrm{n}]$, i.e. the difference between the actual signal amplitude and its round-off value, is the same as with sampling frequency $f_{S}$. It is safe to assume that there is no change in the variance, i.e. in the power, of the quantization errors e $|n|$ and e'[n]:

$$
\left(\sigma_{e^{\prime}}\right)^{2}=\left(\sigma_{e}\right)^{2}
$$

It is also evident that since the sampling interval is $\mathrm{T}_{\mathrm{SO}}=1 / \mathrm{f}_{\mathrm{SO}}$, the power of the quantization error is spread in $\left[-\mathrm{f}_{\mathrm{SO}} / 2,+\mathrm{f}_{\mathrm{SO}} / 2\right]$ frequency band, which is larger that $\left|-f_{S} / 2,+f_{S} / 2\right|$ Nyquist band. Since the power of the error is the same, the power spectral density is smaller by a factor $\mathrm{L}$. 


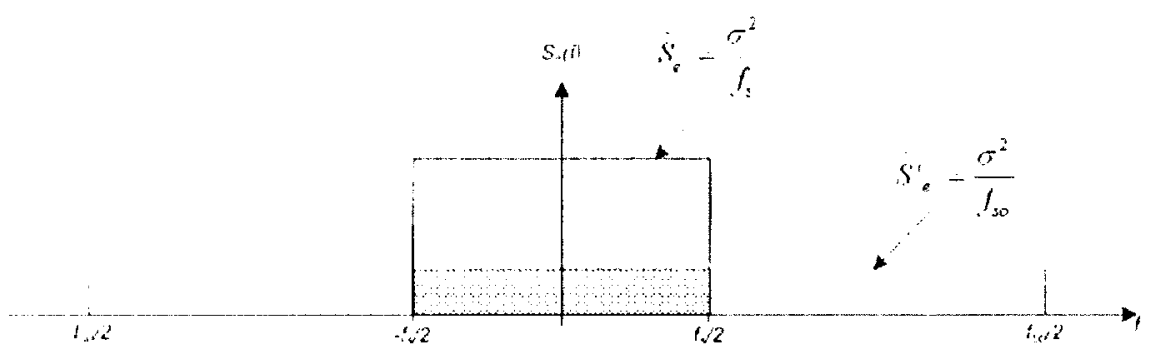

Figure 3.5 Power Spectral Densities of Quantization Errors

As in the case of over-sampling, the signal bandwidth $\left[-f_{S} / 2,+f_{S} / 2\right]$ is not changed, the power of quantization error in the frequency band of interest is smaller by a factor $L$.

$$
\begin{gathered}
S N O R=10 \cdot \log \left(\frac{P_{r}}{P_{e} / L}\right)=6.02 \cdot B+1.76+10 \cdot \log (L) \cdot[\mathrm{dB}] \cdot(3-4) \\
f \in\left[\frac{-f_{s}}{2} \cdot \frac{f_{s}}{2}\right]
\end{gathered}
$$

The over-sampling has another immediate advantage: it relaxes the anti-aliasing filter requirements by allowing a gentle roll-off of the filter. Due to high sampling frequency, the replicas of the signal spectrum at multiples of the sampling frequency are spaced far away from each other, so a large stop-band is allowed for the anti-aliasing filter. Low-order simple LPF's can be used. 

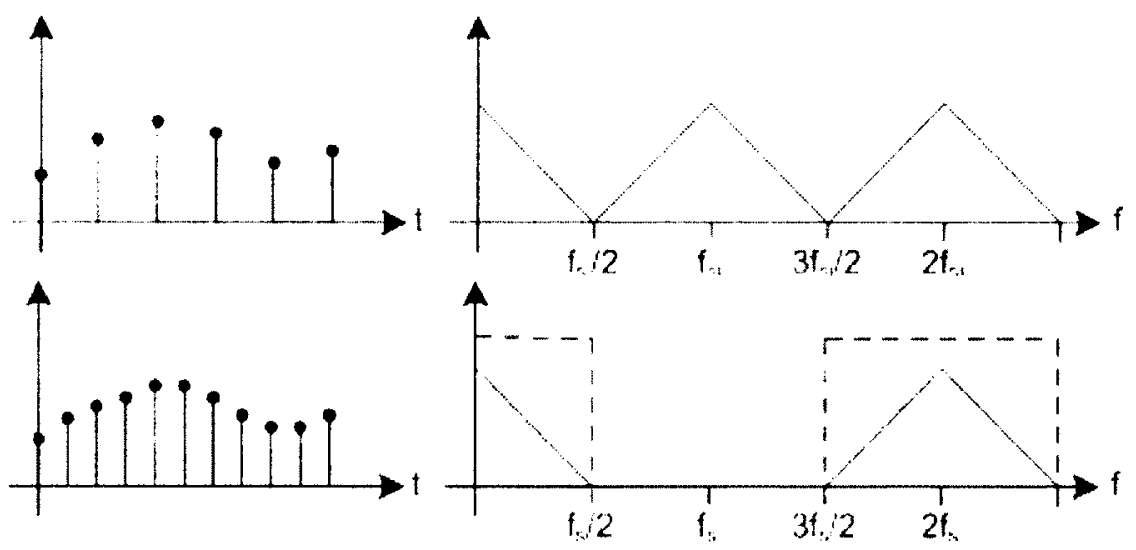

Figure 3.6 Over-sampling Example, $\mathrm{L}=2$

\subsubsection{Signal and Noise Transfer Functions of $\Delta \Sigma$ Modulators}

One of the inherent features of $\Delta \Sigma$ modulators is noise shaping. In combination with the over-sampling, noise shaping allows to further reduce the quantization noise within the band of interest. Noise shaping, as the name implies, involves attenuating the in-band quantization noise at the expense of amplifying noise in the out-of-band region. The resulting spectrum at the output has minimum in-band quantization noise and large out-of-band noise. If a low-pass filter is applied to the output, the out-of-band noise can be eliminated at all, if necessary.

For the sake of analysis the quantization noise is often modeled as a random noise from the generator applied to the output of the modulator as shown in Figure 3.7 below. 


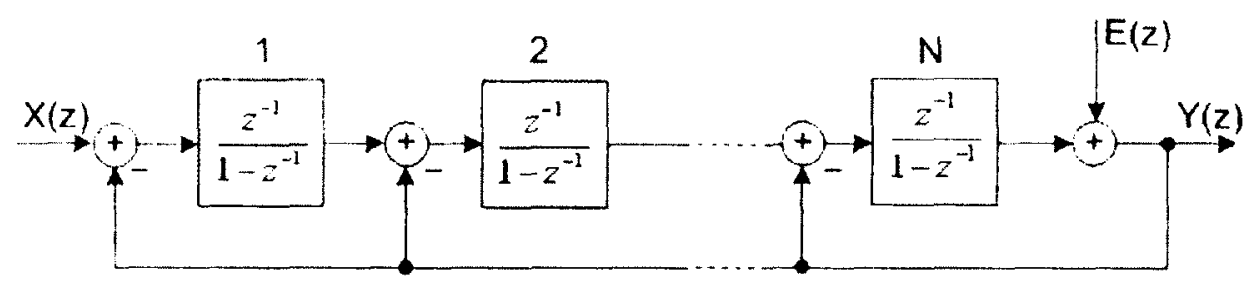

Figure 3.7 N-Order $\Delta \Sigma$ Modulator Functional Block Diagram

Signal and Noise Transfer Functions of $\Delta \Sigma$ modulator depend on its architecture and can be easily determined. For the modulator in Figure 3.7 they are as follows.

$$
S T F=z^{-i}, N T F=\left(1-z^{-1}\right)^{\prime}
$$

Signal to Quantization Noise Ratio for $\Delta \Sigma$ modulator of Nth order is estimated in $\left[10 \mid\right.$. For $2^{\text {nd }}$ Order $\Delta \Sigma$ modulator:

$$
S Y Q R=6.02 \cdot B-11.14+50 \cdot \log (L)
$$

It is worth to note that the Transfer Functions as well as SQNR of $\Delta \Sigma$ modulator depend on its architecture. The STF and NTF (Z-transforms) of the modulators of $1^{\text {st }}$ and $2^{\text {nd }}$ orders are shown in Figure 3.8 below.

The selection of optimum $\Delta \Sigma$ modulator is not straight forward task. $\left(1-Z^{-1}\right)^{N}$ architecture of the $\Delta \Sigma$ modulator is highly prone to instability. It can begin oscillating with $\mathrm{N}=3$ and higher. Thus the modeling and building of stable and yet high-performing modulator is one of most challenging and critical parts of the modulator design.

One of the popular approaches is Multi-Stage Noise Shaping (MASH) structure that is composed of few low-order (and so stable) modulators connected in series. MASH modulators are studied in numerous publications. The modulators resolve the 
stability problem of high-order structures in the expense of more complicated architecture.

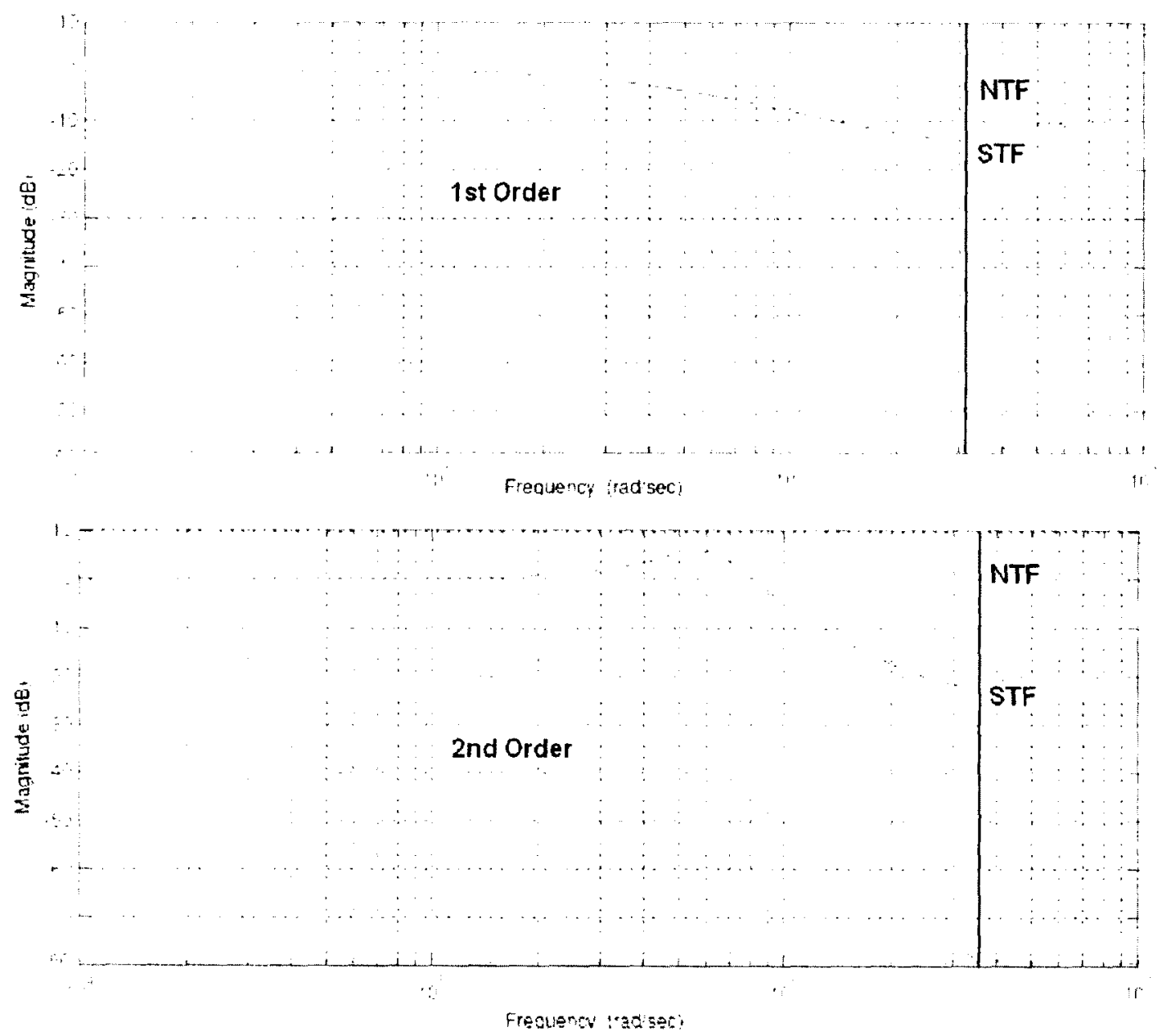

Figure 3.8 Signal and Noise Transfer Functions of Low-Pass $\Delta \Sigma$ Modulators

The $\Delta \Sigma$ modulator in Figure 3.7 above is classified as low-pass modulator. Lowpass modulator can be modified to band-pass by replacing the integrator $z^{-1} *\left(1-z^{-1}\right)^{-1}$ for the resonator with transfer function $z^{-2 *}\left(1-z^{-2}\right)^{-1}$. 


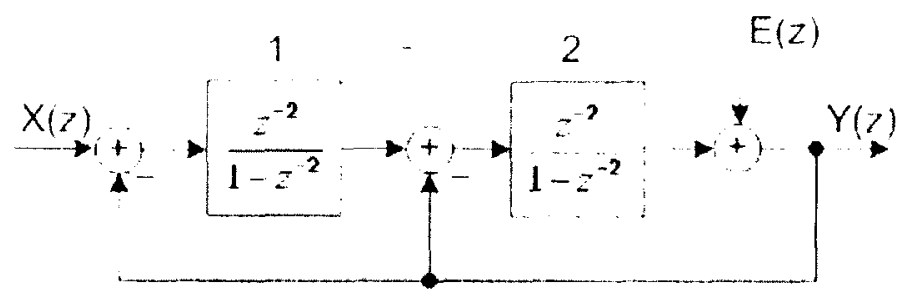

Figure 3.9 Band-pass $\Delta \Sigma$ Modulator of 2nd Order

Band-pass $\Delta \Sigma$ modulators maintain the advantages of their low-pass counterparts, i.e. reduced quantization error due to over-sampling and noise shaping. The central frequency and bandwidth of the modulators depends on over-sampling frequency value, the order and architecture of the modulator.

The publications related to band-pass $\Delta \Sigma$ modulators discuss mostly the devices with signal central frequency $2-10 \mathrm{MHz}$ and bandwidth up to $200 \mathrm{kHz}[12,13,14]$. These modulators are very convenient for digitizing narrowband IF signals. At the same time, they are not suitable for DSL applications, which spectra are located close to the base band, and the bandwidth can cover few $\mathrm{MHz}$ range.

Further study will consider low-pass $\Delta \Sigma$ modulators only.

\subsection{Practical Implementations of $\Delta \Sigma$ Modulators}

Switched-capacitor (SC) technique is dominated approach in the design of discrete-time $\Delta \Sigma$ modulators. The technique uses linear on-chip capacitors to store a signal (or charge). By controlling switches and employing operational amplifiers, the charges can be manipulated and shifted from one capacitor to the other. 


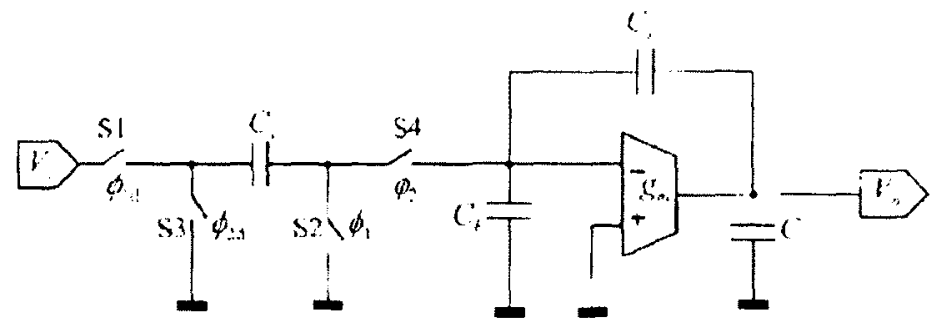

Figure 3.10 Switched Capacitor Integrator

SC based $\triangle \Sigma$ modulators exhibit performance that meets ADSL specifications with regards to signal-noise ratio and signal dynamic range. In $|15|$ the authors suggest the $\triangle \Sigma$ ADC solution that uses MASH 2-1-1 cascaded topology with optimized coefficients. The ADC generates one-bit signal and can be used as a modulator for the line driver. For over-sampling ratio of only 24 , the converter achieves SNR of $87 \mathrm{~dB}$ and input dynamic range of 15 bits. The converter is sampled at $52.8 \mathrm{MHz}$ and implemented in $0.5 \mu \mathrm{m}$ CMOS technology. The $\Delta \Sigma$ modulator of similar performance is reported in [16]. The device is sampled at $70.4 \mathrm{MHz}$ and implemented in $0.25 \mu \mathrm{m}$ CMOS technology.

Advanced sub-micron technologies and, as a result. lower voltage supply headroom force designers to employ more complex topologies of $\Delta \Sigma$ modulators/converters and use higher sample rates $[17,18]$.

Switched-current (SI) technique is the alternative approach in design of $\Delta \Sigma$ modulators. SI integrator is shown in Figure 3.11 below 1191 . The SI method uses current rather than voltage to represent signal. It doesn't require linear on-chip capacitors, and so standard CMOS process without extra technological steps can be used. 


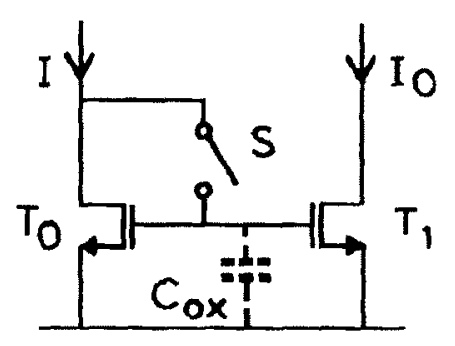

Figure 3.11 Switched Current Integrator

SI technique was introduced in early 90 s $[19,20]$. Potentially, a current domain operations offer greater ease for signals algebraic manipulation and allow lower power supply voltages compared to voltage domain operations.

High-sampling frequencies and low voltage supply make SI $\Delta \Sigma$ modulators well suited for advanced submicron technologies. That is why most low-power and lowvoltage designs of $\Delta \Sigma$ modulators are based on SI technique. However, SI-based modulators often have lower figures of merits $[21,22,23]$. Just few designs of SI $\Delta \Sigma$ modulators exhibit the performance of the same level as of SC-based modulators. For example, in $\mid 24\}$ the authors declare dynamic range of 12 bit and signal-noise-ratio of $80 \mathrm{~dB}$.

A comparative study of SC and SI integrators that are the main functional units of $\Delta \Sigma$ modulators $|25|$ revealed that with early CMOS technologies SC circuits performed much better than their SI counterparts. However, as the technologies head towards low power supply voltages, the performance of SC circuits drops steadily while that of SI remains almost constant. It is expected that with advanced CMOS 
technologies the performance of SI circuits will match and eventually surpass the performance of SC circuits.

$\Delta \Sigma$ modulators, either SC or SI, as they are presented in most publications, are made as analog circuits with analog inputs. The modulators are convenient for analogto-digital conversion and well fit a receiver path. As for the transmission circuitries the input signal is not necessary to be analog. This is particularly applicable for DSL line drivers. The output of IFFT processor in Figure 2.4 is digital. If so, it is reasonable to implement the $\Delta \Sigma$ modulator for the $T x$ path as pure digital circuit.

Digital implementation of $\Delta \Sigma$ modulator has certain advantages. The main of them is that a digital circuit is well suited for most CMOS technologies. Timing parameters of circuit components (signal rise/fall time, delay) appear to be essential only as no analog signals are used at all. The advanced CMOS technologies are very attractive for such applications as they allow higher sample rates and less signal delays. Of course, it is obvious that all internal operations used in the $\Delta \Sigma$ modulator (delay, amplification, signals adding/subtraction) can be done digitally in discrete time domain.

Existing tools such as Matlab and Simulink provide very convenient and fast way to synthesize any $\Delta \Sigma$ modulator topology at a system level and then to simulate its performance. The HDL Coder, which is part of Simulink design environment, can be used to generate the HDL code that is suitable for FPGA or ASIC implementations. This essentially reduces the design time and makes the development of the $\Delta \Sigma$ modulator a routine procedure. 


\subsection{Selection of $\Delta \Sigma$ modulator Architecture for ADSL}

\section{SLD}

The $\Delta \Sigma$ modulator as it is used in DSL transmitter chain converts multi-bit signal from IFFT module to single-bit data stream that is suitable for switching power amplifier. The $\Delta \Sigma$ modulator should meet the requirements defined in $[7,8,26]$. The main of them, that are applicable for $\Delta \Sigma$ modulator design, are outlined below.

- DMT sub-carriers spacing $\Delta \mathrm{f}=4.3125 \mathrm{kHz}$.

- Max number of sub-carriers is $\mathrm{N}=256$.

- Max number of bits per sub-carrier supported by the transmitter $8 \leq \mathrm{Bit}_{\text {max }}$ $\leq 15$.

- $\quad$ Missing Tone Power Ratio MTPR $\geq\left(3 \times \mathrm{Bit}_{\max }+20\right) \mathrm{dB}$

Each sub-carrier is quadrature-amplitude modulated with modulation order from 64 to 256 (64-QAM through 256-QAM). The MTPR is measured as a ratio of the RMS value of the tone at some sub-carrier frequency to the RMS value of all the non-tone signals in $\Delta \mathrm{f}=4.3125 \mathrm{kHz}$ frequency band centered on that sub-carrier frequency.

MTPR varies from $44 \mathrm{~dB}$ for 64-QAM to $65 \mathrm{~dB}$ for 256-QAM. 


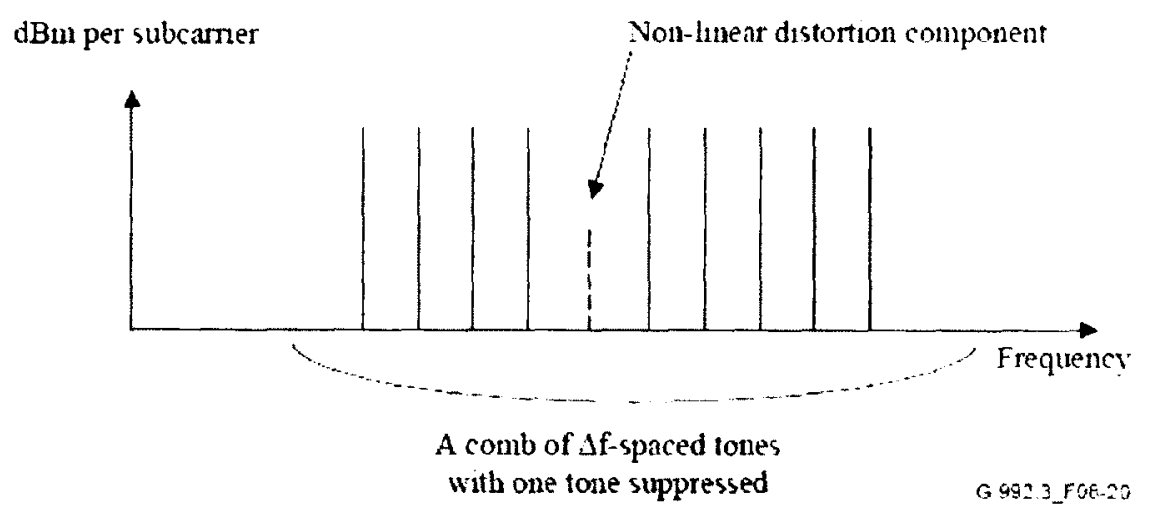

Figure 3.12 Missing Tone Power Ratio [24]

There are few power spectrum masks defined in G.992.1 [7] and G.992.2 [8] documents. The mask shown in Figure 2.1 above outlines the ADSL signal spectrum for non-overlap up- and down-spectrum operations.

$\Delta \Sigma$ modulators are studied by many authors using Matlab and Simulink. These tools provide very convenient and effective way to build any $\Delta \Sigma$ modulator's architecture and then to simulate it. The method and tool box presented in [27] is used in this work to characterize the $\Delta \Sigma$ modulator for ADSL applications. The models discussed in [27] are designed mostly for audio applications. The model studied in this work is adjusted to match specific ADSL requirements, in particular, related to the signal bandwidth.

Based on the subject analysis, $2^{\text {nd }}$ order low-pass $\Delta \Sigma$ modulator topology is selected as an initial model for ADSL application. The topology is unconditionally stable and has signal and noise transfer functions shown in Figure 3.8 above that are suitable for ADSL bandwidth.

Simulink model of the $\Delta \Sigma$ modulator is shown in Figure 3.13 below. 


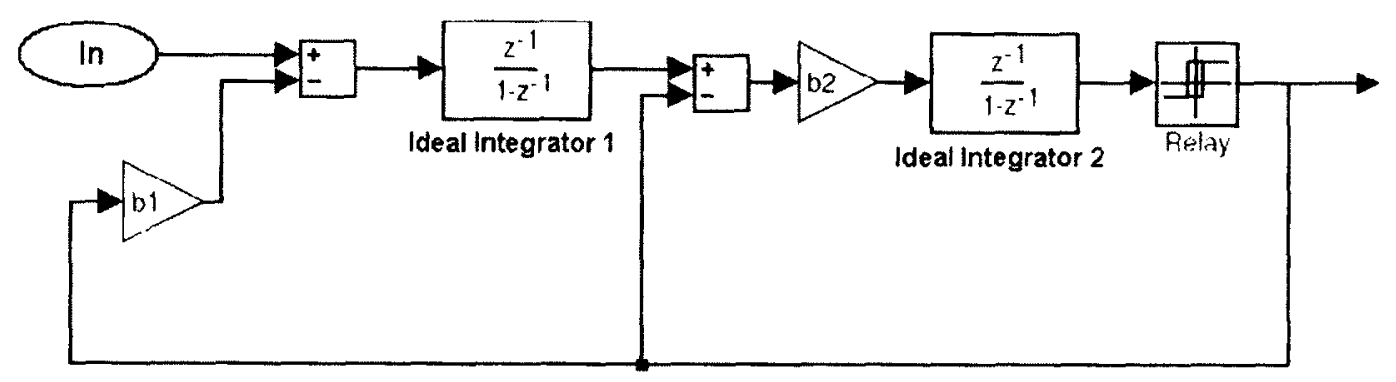

\section{Figure 3.13 Simulink Model of $2^{\text {nd }}$-order $\Delta \Sigma$ Modulator}

The input signal of the model is analog. Single-tone sine wave is usually applied at the input to evaluate the distortion of the signal passed through the modulator. The signal at the model's output takes one of two possible states, which levels are defined by the Relay module. The output can be connected to the following switching line driver. The high voltage at the output can be treated as logical-high signal; accordingly, the low voltage corresponds to logical-low signal. At the same time, the feedback signal is analog.

It is worth to note that the analog signals in Matlab/Simulink are usually represented by 64-bit digital code.

The output signal is analyzed with FFT processor in order to determine the level of signal distortion. The FFT processor itself introduces some level of errors/noise due to the finite number of signal samples collected at the measurement interval, which is limited in time. To minimize the processing errors, the number of input signal samples $\mathrm{N}=65536$, the number of sine-wave periods $\mathrm{N}_{\mathrm{PER}}=128$ and the value of Over-Sampling 
Ratio $\mathrm{OSR}=128$ are selected to be multiple of 2 . The OSR is supposed to be high enough to benefit from noise shaping feature of the $\Delta \Sigma$ modulator.

The sample frequency and input signal frequency are calculated using the following expressions:

- $\mathrm{f}_{\mathrm{S}}=2 \cdot \mathrm{OSR} \cdot \mathrm{BW}=128 \mathrm{MHz}$, where $\mathrm{BW}=500 \mathrm{kHz}$ is the signal processing bandwidth;

- $\quad \mathrm{f}_{\mathrm{IN}}=\mathrm{N}_{\mathrm{PER}} \cdot \mathrm{f}_{\mathrm{S}} / \mathrm{N}=250 \mathrm{kHz}$.

Although the OSR value is relatively high, the sampling frequency $f_{S}=128$ $\mathrm{MHz}$ is reasonable for most CMOS technologies that can be used to implement the $\Delta \Sigma$ modulator in silicon.

It is worth to note that the BW parameter above is not the model's operational characteristic, but the value, which defines, together with OSR, N, NPER values, the parameters of the algorithm that processes the results of the model simulation. The processing bandwidth defines the noise power that is used to calculate the Dynamic Range (DR) of the modulator. By definition, the DR is a ratio of distortion-free maximum signal power to the noise power within the processing bandwidth. The dynamic range can be roughly estimated as Signal-Noise Ratio (SNR) provided that no signal distortion products are observed within the processing bandwidth.

The Dynamic Range is close to the Signal-Noise Distortion Ratio (SNDR), which is used for the modulator's evaluation when quantization noise is taken into account only. The SNDR can be estimated for single-bit $\Delta \Sigma$ modulator as follow [28]. 


$$
\operatorname{SNDT}_{\text {natax }}=\frac{3 \pi}{2} \cdot(2 n+1) \cdot\left(\frac{\text { OSR }}{\pi}\right)^{(2 n+1)}(3-7)
$$

where $\mathrm{n}$ is the order of the modulator. The calculation shows that the SNDR value for the modulator model in Figure 3.13 above $(n=2, \operatorname{OSR}=128)$ is expected to be $\mathrm{SNDR}=94 \mathrm{~dB}$.

The model of the $\Delta \Sigma$ modulator in Figure 3.13 has its intrinsic parameters that have been adjusted during simulation to get the highest possible signal-noise ratio and accordingly the DR at the output of the modulator. The model's parameters are as follows.

- $\quad$ Gain coefficient $b l=0.5$

- Gain coefficient $b 2=0.5$

- Saturation voltage of integrators $\mathrm{V}_{\mathrm{SAT}}=1.5 \mathrm{~V}$

- Amplitude voltage at Relay output $\mathrm{V}_{\mathrm{REF}}=1 \mathrm{~V}$

- Input signal amplitude $\mathrm{V}_{\mathrm{IN}}=0.175 \mathrm{~V}$.

$\mathrm{V}_{\mathrm{SAT}}$ and $\mathrm{V}_{\mathrm{REF}}$ voltages represent the parameters of the model's components and are generated internally. Input signal is provided by the Simulink component, which is not shown in Figure 3.13 above.

The modulator signals after $1^{\prime \prime}$ adder, $1^{\text {st }}$ integrator, $2^{\text {nd }}$ integrator and at the output of the model are shown in Figure 3.14 below. In the screenshots below the horizontal axis represents time in seconds, the vertical axis represents the signal amplitude in volts. 


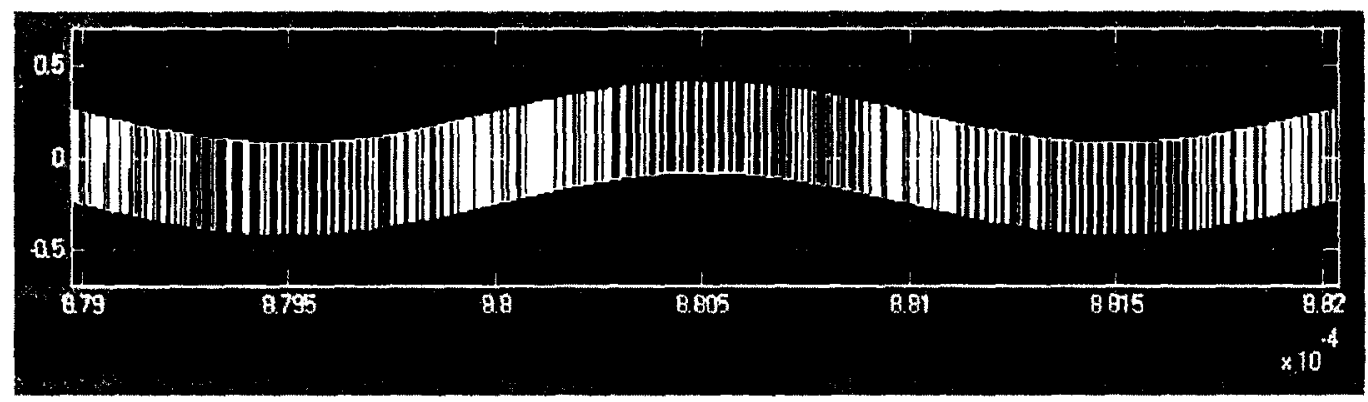

a) Adder-subtractor Output

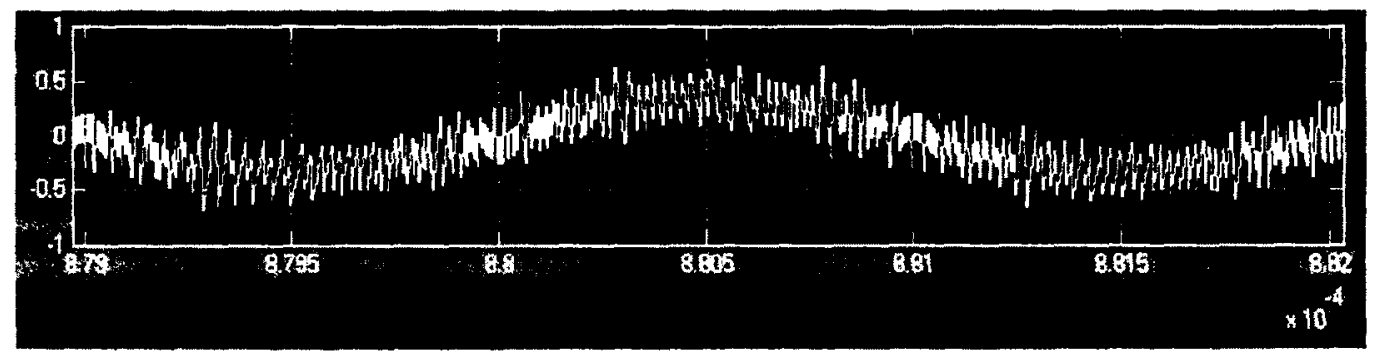

b) 1st Integrator Output

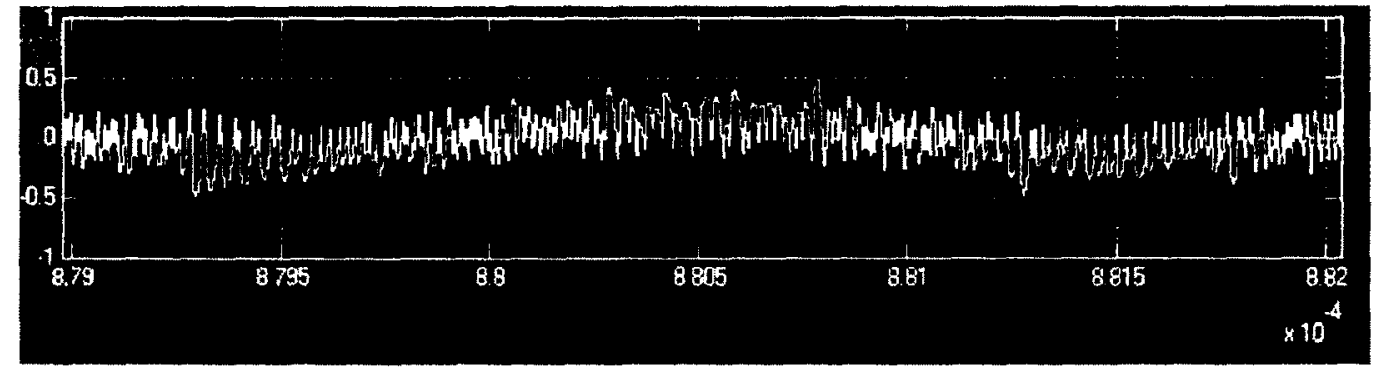

c) 2nd Integrator Output

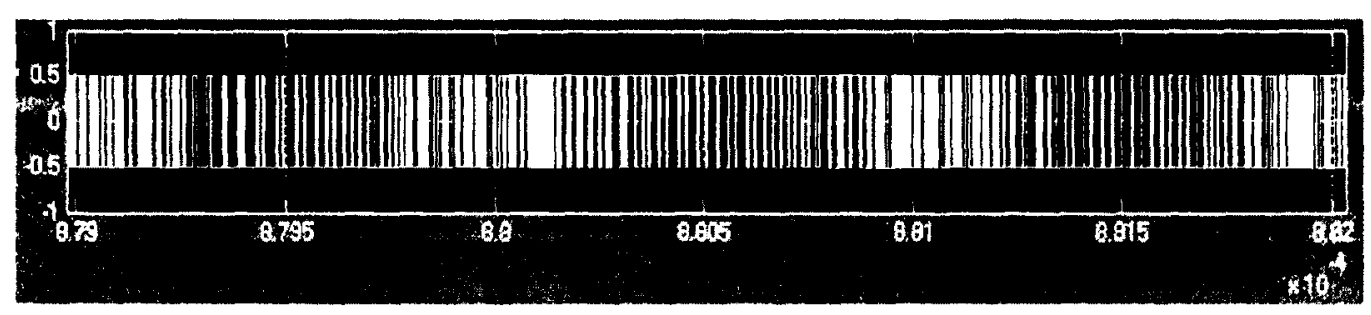

d) Relay Output

Figure 3.14 Internal Signals of 2nd Order $\Delta \Sigma$ modulator

As said above, the output signal of the modulator is processed with FFT algorithm to estimate the distortion of the signal passed through the modulator. The Matlab scripts that implement the FFT processing are based on $m$ files from the 
Toolbox developed by P. Malcovati. The Toolbox suite is available at Matlab Central File Exchange. The original Matlab scripts from the Toolbox are modified to address the parameters of $2^{\text {nd }}$-order $\Delta \Sigma$ modulator designed for the ADSL application. The modified Matlab scripts are provided in Appendix A.

The simulation results are depicted in Figure 3.15 and Figure 3.16 below. The dynamic range is identified as SNR value in Figure 3.15. The simulation proves that $2^{\text {nd }}$-order $\Delta \Sigma$ modulator has the dynamic range at least $85 \mathrm{~dB}$. This value is almost 10 $\mathrm{dB}$ less than theoretical SNDR=94 $\mathrm{dB}$. However, the $\mathrm{DR}$ is high enough, and the modulator can be used in the transmission chain supporting the data capability of more than 14 bits per carrier.

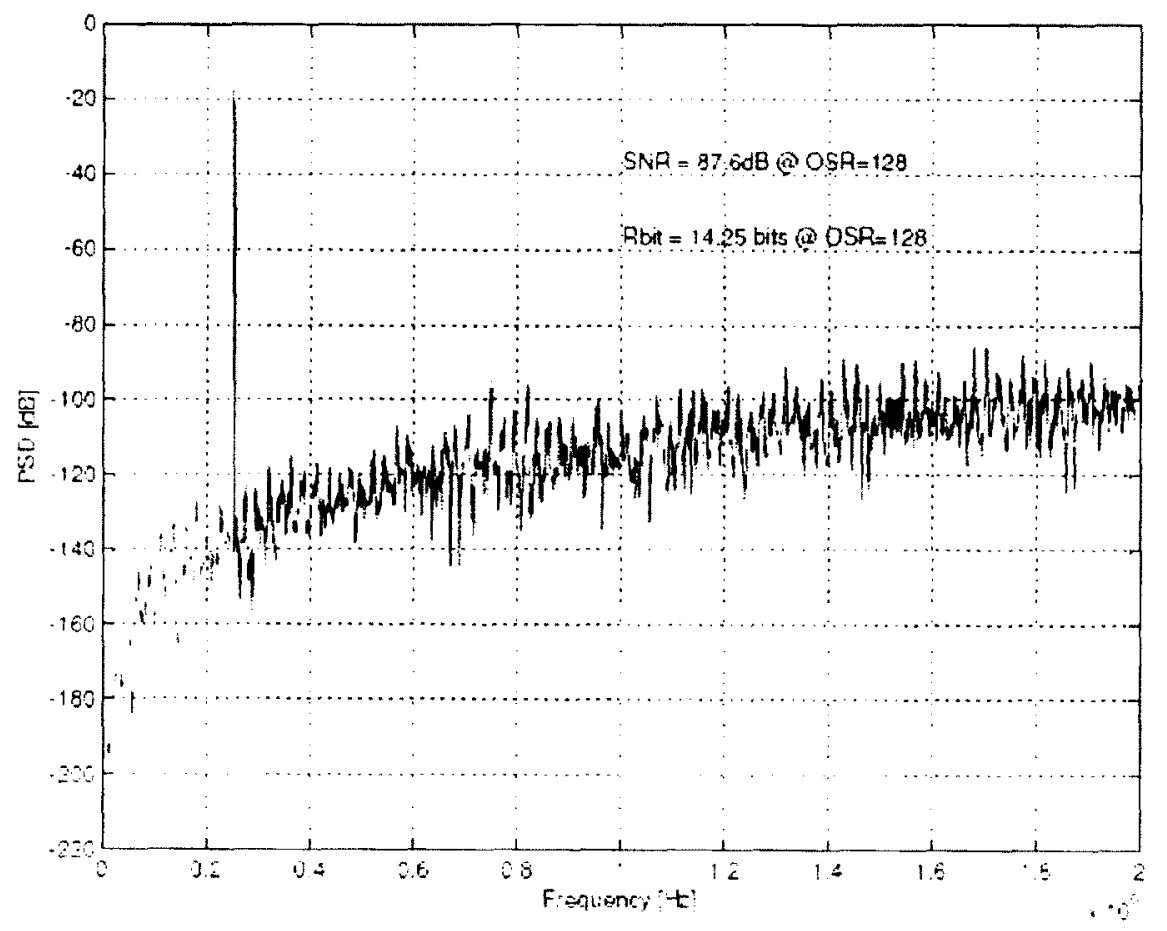

Figure 3.15 Signal Spectrum of $2^{\text {nd }}$-order $\Delta \Sigma$ Modulator's Simulink Model, $\mathrm{f}_{\mathrm{IN}}=250 \mathrm{kHz}$ 


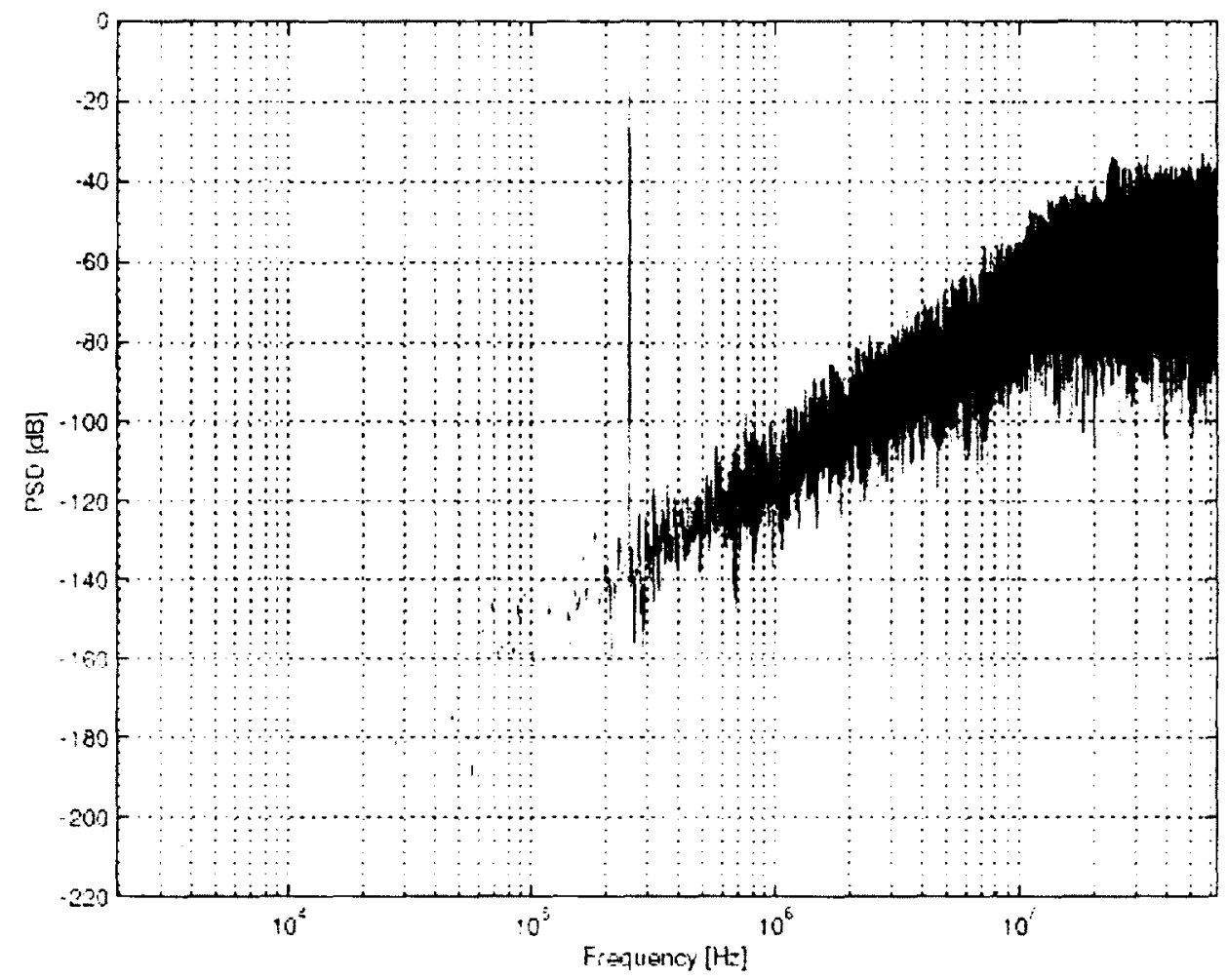

Figure 3.16 Signal Spectrum of $2^{\text {nd }}$-order $\Delta \Sigma$ Modulator's Simulink Model, $\mathrm{f}_{\mathrm{IN}}=250 \mathrm{kHz}$

MTPR value at the output of the $\Delta \Sigma$ modulator is evaluated with the Simulink toolbox shown in Figure 3.17 below.

The toolbox includes base-band DMT Modulator block available in Simulink Demo Library. The DMT Modulator is comprehensively discussed in 16|. The Modulator is developed to study the performance of ADSL communication system and is ideally suitable for the MTPR evaluation. The Modulator generates 256 orthogonal sub-carriers, which are modulated by the data stream from stochastic data generator. Sample rate of the generator is $4000 \mathrm{sec}^{-1}$ that corresponds to symbol update rate $4 \mathrm{kHz}$. per sub-carrier. This value is close to ADSL symbol rate $f_{S}=4.3125 \mathrm{kHz}$ and is selected 
just for the convenience of further processing. The symbol rate defines the space between the sub-carriers and, so the overall bandwidth occupied by the DMT signal is going to be smaller with smaller symbol rate. However, such change is not essential for the purpose of this work.
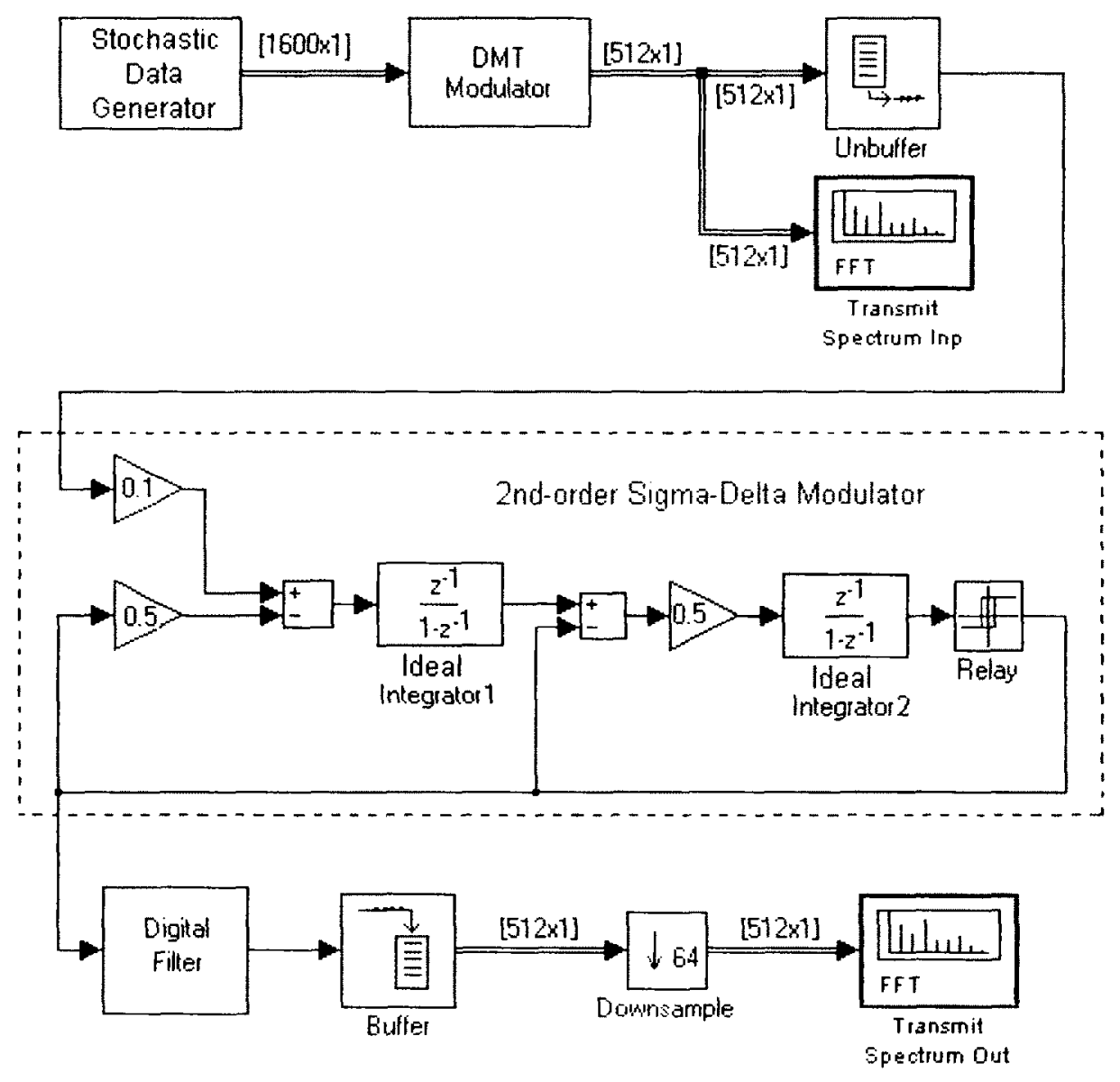

Figure 3.17 MTPR Test Toolbox

Transmit Spectrum Inp Scope of the MTPR toolbox is used to capture the spectrum of the signal generated by the DMT Modulator. The data from the DMT Modulator has frame-based structure that is necessary for FFT processing. As the $\Delta \Sigma$ modulator accepts unbuffered data only, Unbuffer module is inserted in between the 
DMT and $\Delta \Sigma$ modulators. The signal from the Unbuffer module is wired to the input of the $2^{\text {nd }}$-order $\Delta \Sigma$ modulator. The architecture and parameters of the $\Delta \Sigma$ modulator in the MTPR toolbox in Figure 3.17 are the same as of the modulator shown in Figure 3.13.

The output signal of the $\Delta \Sigma$ modulator is passed through $5^{\text {th }}$-order digital lowpass filter with bandwidth $\mathrm{BW}=1 \mathrm{MHz}$ to reduce the contribution of out-of-band noise in the calculated spectrum of the output signal. The spectrum is captured by Transmit Spectrum Out Scope.

Sampling frequency of the MTPR toolbox $\mathrm{f}_{\mathrm{S}}=132.072 \mathrm{MHz}$ is multiple of data sample rate of stochastic data generator $D_{S}=4000$ bits $/ \mathrm{sec}$, number of DMT subchannels $N=256$ and of power of 2 . The latter is necessary to maintain high resolution of Transmit Inp and Out Spectrum Scopes, which employ FFT algorithm.

The input data is distributed over the sub-channels in the way as it is usually done in the ADSL systems. For each sub-channel, different number of bits is assigned according to the SNR measured at corresponding sub-carrier. This defines the QAM order at the sub-carrier. The bits distribution for each sub-channel used in the MTPR toolbox is depicted in Figure 3.18 below. The maximum load per sub-channel is 12 bits.

To estimate the Missing Tone Power Ratio of the signal at the output of the $\Delta \Sigma$ modulator few sub-carriers in the DMT modulator are disabled. Signal power level at the "empty" spots is used to estimate the noise due to intermodulation products produced by the $\Delta \Sigma$ modulator. The depth of the cutout in the Power Spectrum Density function actually represents the MTPR value. 


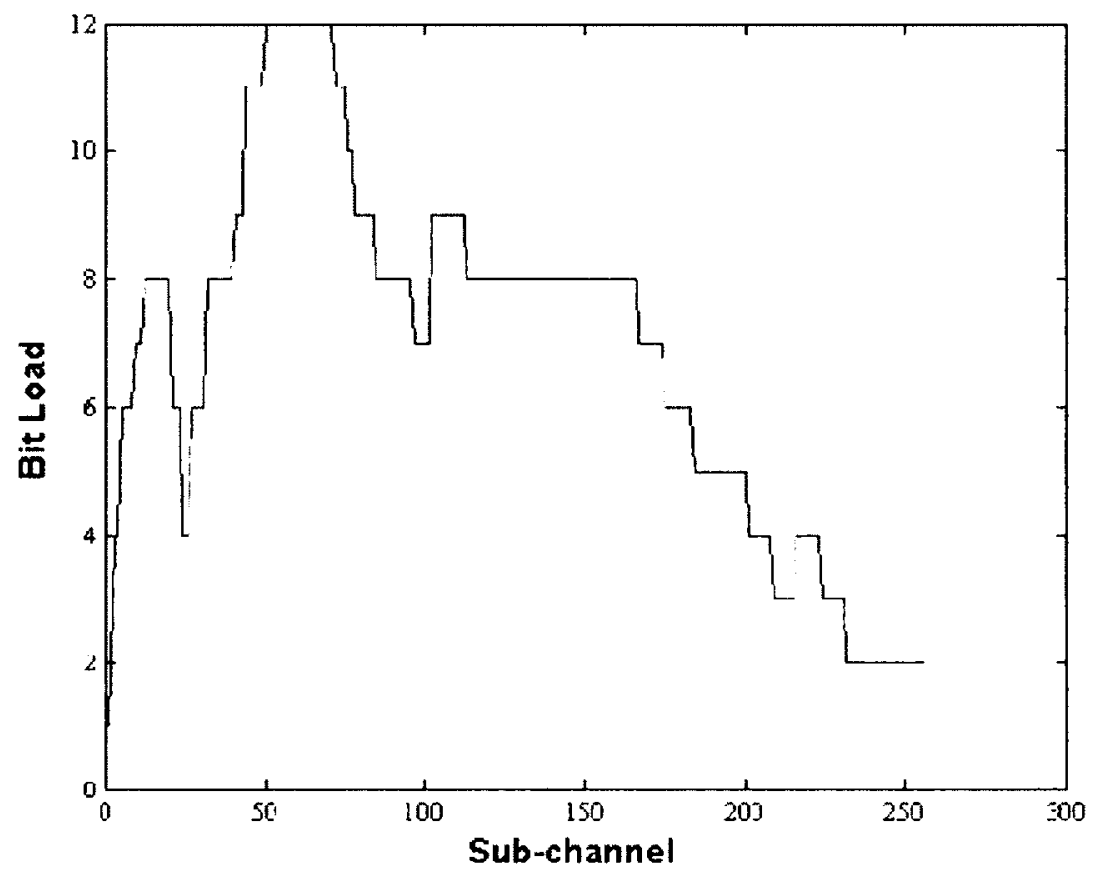

Figure 3.18 Bits Loading Distribution

The results of model simulation are shown in Figure 3.19 below.

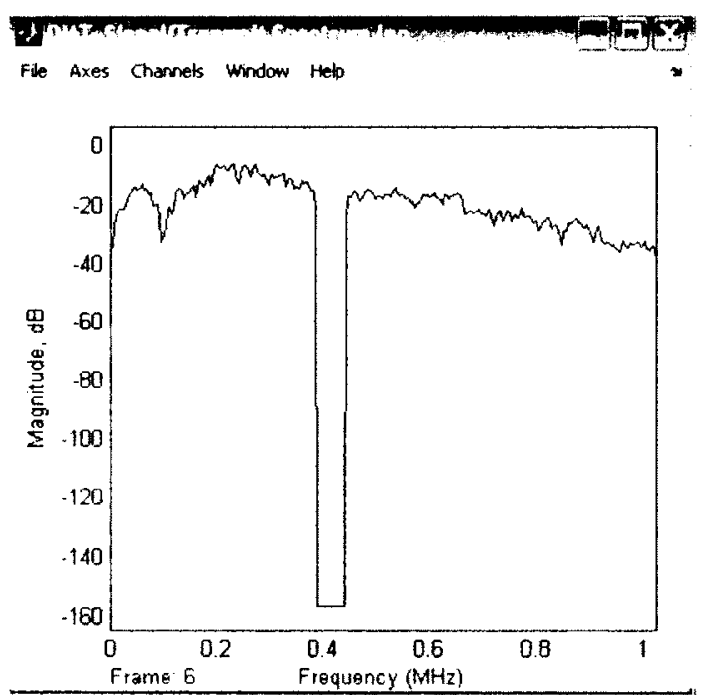

a) Input Spectrum

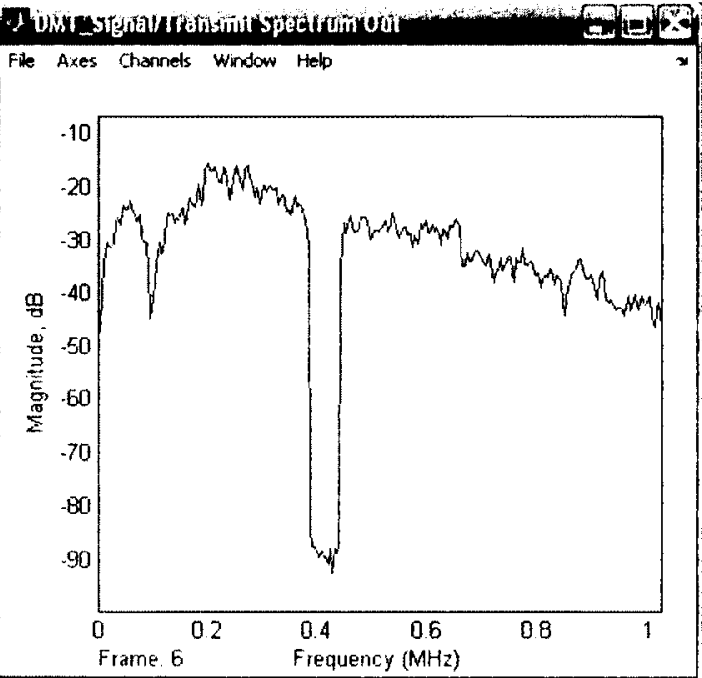

b) Output Spectrum

Figure 3.19 Signal Spectra at the Input and Output of 2 nd-Order $\Delta \Sigma$ Modulator 
The MTPR value of the signal at the input of the $\Delta \Sigma$ modulator is about $140 \mathrm{~dB}$ (Figure 3.19, a). When the signal is passed through the modulator, the MTPR degrades to about $65 \mathrm{~dB}$ (Figure $3.19, \mathrm{~b}$ ). This demonstrates the $2^{\text {nd }}$-order $\Delta \Sigma$ modulator meets ADSL requirements.

\subsection{Conclusion}

Matlab/Simulink simulations show that $2^{\text {nd }}-$ order $\Delta \Sigma$ modulator is suitable as the data encoder for ADSL line drivers. $\Delta \Sigma$ modulators of higher orders will probably improve the MTPR value and signal dynamic range. However, implementation of highorder modulators will certainly bring extra complexity that will definitely jeopardize the potential benefits of high-order models of $\Delta \Sigma$ modulators.

$2^{\text {nd }}$-order $\Delta \Sigma$ modulator will be used below as the modulator for ADSL line driver. 


\section{Switching Power Amplifier as a Potential}

\section{ADSL Line Driver}

Single-bit-output $\Delta \Sigma$ modulator is self-sufficient circuitry that can produce the signal in the format required for the ADSL communication link. The only signal parameter that might be not matching the relevant specifications is the signal strength.

ITU G.992.1 [7] and G.992.2 [8] documents provide the recommendations for the ADSL signal power levels at the line terminals. Aggregate power of Central Office transmitter (ATU-C) should be no more than $20.4 \mathrm{dBm}$ across the whole down-link bandwidth $138 \mathrm{kHz}-1104 \mathrm{kHz}$. The power of Customer Premise transmitter (ATU-R) should be no more than $13 \mathrm{dBm}$ across the up-link bandwidth $25.875 \mathrm{kHz}-138 \mathrm{kHz}$. The power levels maintained in the ADSL transmitters are significant for digital components regardless the technology used to fabricate them.

In case of $\Delta \Sigma$ modulator the data to be sent is encoded by signal level transitions at certain moments of time. Obviously, the power amplifier shouldn't destroy the signal appearance in time domain. Switching or Class D power amplifiers are the best suited for such a task. It is worth to note that signal amplitude dynamic range is not a matter of concern for switching amplifier. The signal amplitude can be recovered at the receiver side by passive low-pass filter, and so the dynamic range doesn't depend on the available voltage supply headroom anymore. Of course, the voltage supply defines the parameters of the circuitry, but in different manner. 
Standard CMOS inverter with proper transistors dimensions could be a natural solution for ADSL line driver when $\Delta \Sigma$ modulator is used. The inverter as a line driver amplifier is studied in numerous publications. $[3,29,30]$ are among them. The authors investigated potential causes of signal degradation at the output of the inverter when it is used as an amplifier. The main of them are:

- Power loss due to parasitic capacitances in CMOS devices;

- Large current and high power loss as a result of this when low power supply voltage is used;

- Large current spikes happening at the moment of signal level switching.

The following discussion addresses these and other issues that appear to be essential for the subject of this work.

\subsection{The Discussion of Class D Power Amplifier}

Class D power amplifier is one of the implementations of more generic switching power amplifier.

Class D amplifier is an electronic circuit where power devices (usually MOSFETs) are operated as binary switches. The switches are either fully ON or fully OFF with minimum time spent between these two stages. Regardless the digital-like mode of operation the amplifiers deal with analog input signals and produce analog signals at their output terminals. The bandwidth of these signals are usually well below the switching frequency of amplifier's power devices.

Generic block diagram of Class D power amplifier is given in Figure 4.1 below. 


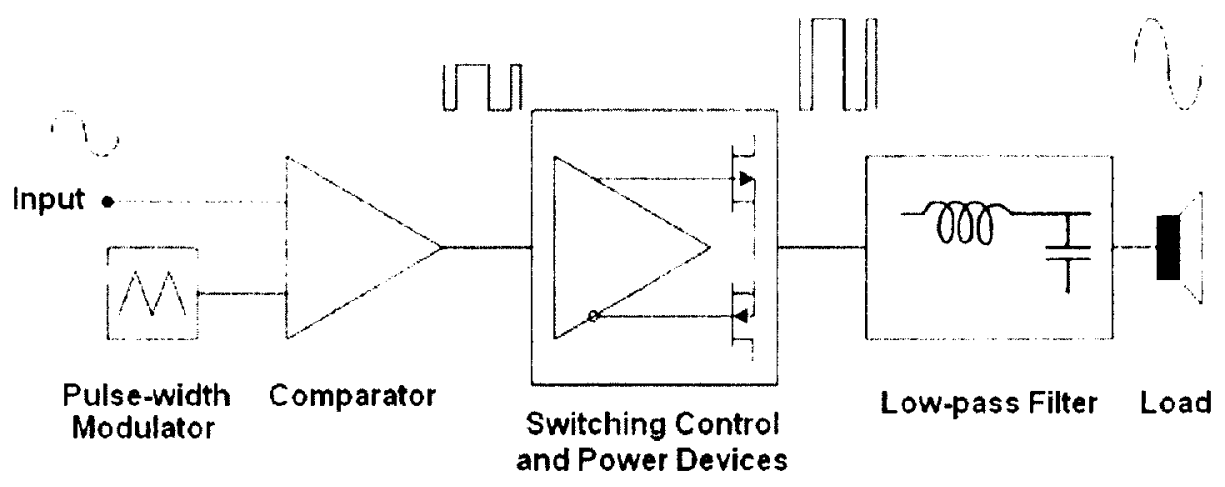

Figure 4.1 Class D Power Amplifier

The amplifier works by generating a square-wave signal with the spectrum, which low-frequency portion is essentially the wanted output signal. The highfrequency portion of the spectrum is the result of signal transformation to digital-like form so the signal can be amplified by switching power devices.

Class D amplifiers are well known for their high efficiency, in particular, in audio applications. Theoretical power efficiency of Class D amplifier is $100 \%$, if switching devices are taken into account only. This is because an ideal switch doesn't contribute to power losses at all. The switch has zero resistance in ON state, and so no heat is dissipated. In OFF state, the switch conducts no current, and no heat dissipation takes place again.

Real power MOSFET devices are not ideal switches. Their ON resistance is low, but not zero, so some signal power is converted in heat. Current leakage takes place in OFF state of the devices as well. Such switch non-ideality causes some degradation of the amplifier efficiency. 
The power efficiency of Class $\mathrm{D}$ amplifiers degrades also due to passive lowpass filter connected to the output terminals of switching power devices. The filter removes unwanted high-frequency portion of the square-wave signal spectrum to recover low-frequency signal of interest. As some power is consumed to amplify wideband signal, filtering out of high-frequency components of this signal after the amplification brings the power efficiency of the whole unit down. This is regardless the fact that low-pass filter is usually pure reactive circuit made of inductors and capacitors. The efficiency degradation due to low-pass filtering can be reduced by increasing the switching frequency. When the frequency is high enough, high-order signal harmonics produced by the switching are allocated far away from the main signal component and have relatively low strength. High switching frequency allows also using low-pass filters of lower order as their attenuation of high-order harmonics appears to be sufficient to recover the signal of interest with necessary accuracy.

The practical efficiency of the Class D power amplifiers is around $90 \%$ in audio applications. This is well above theoretical efficiency of Class A, B, AB amplifiers.

\subsection{Efficiency of Switching Power Amplifiers with}

\section{Matching Circuit}

One of the main advantages of switching power amplifier is its high efficiency mostly due to low ON resistance of output power devices. This might make necessary to use matching circuit to get maximum possible power transfer from the amplifier to the communication line. 
Maximum power transfer theorem, also referred as Jacobi's law, states that, to obtain maximum external power from a source with finite internal resistance, the resistance of the load should be equal to the resistance of the source viewed from the output terminals. The condition of maximum power transfer doesn't result in maximum efficiency.
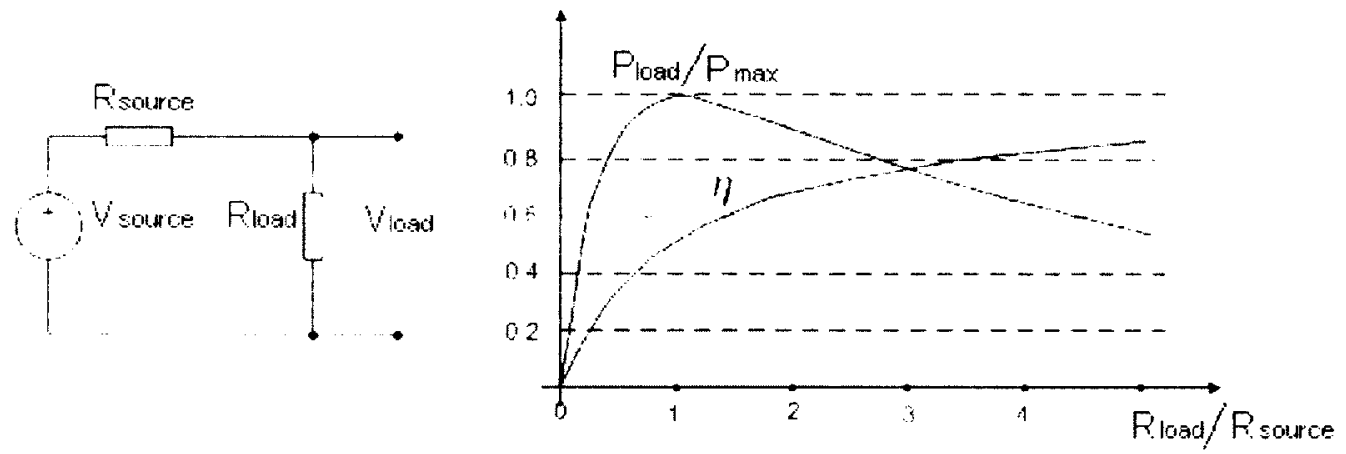

Figure 4.2 Power Transfer Efficiency

If the efficiency $\eta$ is defined as the ratio of power dissipated by the load to power developed by the source, then it is easy to find out from the circuit in Figure 4.2 that the efficiency can be calculated as follow.

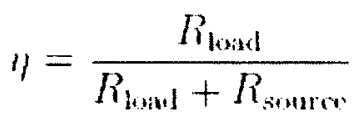

Three particular cases can be considered:

- $\eta=0$, when $R_{\text {load }}=0$;

- $\eta=0.5$, when $R_{\mathrm{load}}=R_{\text {source }}$

- $\eta=1$, when $R_{\text {load }}=\infty$ or $R_{\text {source }}=0$. 
The efficiency is only $50 \%$ when maximum power transfer is achieved, but comes to $100 \%$ as the load resistance approaches infinity, though the total power level changes towards zero. Efficiency also approaches $100 \%$ if the source resistance is reduced to zero. All the above applies to resistive (real) component of the power only.

Equivalent electrical diagram of switching power output stage is as follow.
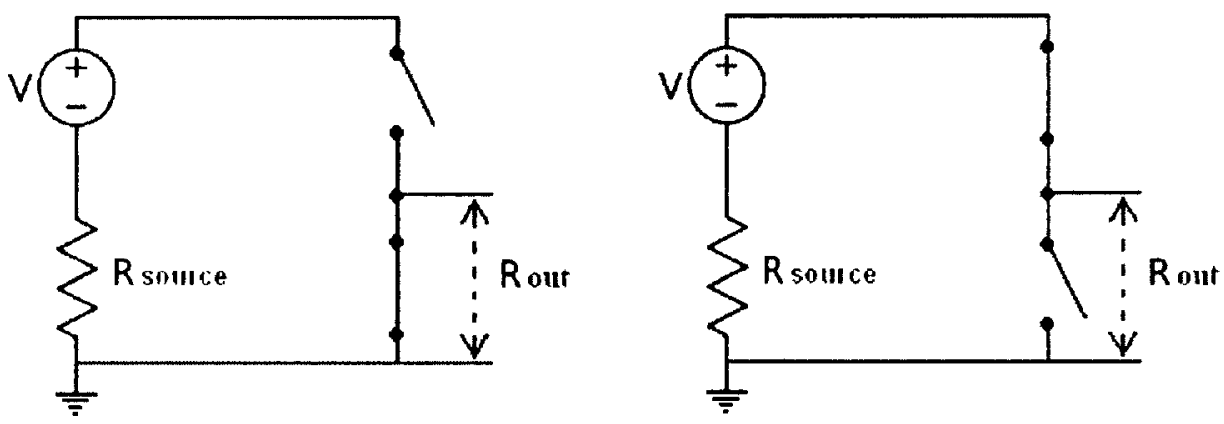

Figure 4.3 Equivalent Electric Diagram of Switching Amplifier

As one can see in Figure 4.3 above, the output impedance of the amplifier is equal to:

$$
R_{\mathrm{oul}}=R_{\text {switch }}+R_{\text {source }}
$$

where $R_{\text {switch }}$ is the $\mathrm{ON}$ resistance of the closed switch, $R_{\text {source }}$ is internal resistance of power supply. The power supply of the circuit is usually a voltage source, which has low internal resistance. This means that the output impedance of switching amplifier is relatively low.

Class D power amplifiers are initially employed for the amplification of atudio signals. The load of audio amplifiers is a speaker with low input impedance. Speaker impedance is usually from less than one ohm to tens ohms depending on the capacity of 
the speakers. These values are matching with the output impedance of switching MOSFET devices in closed state.

One of the key factors that defines the impedance of MOSFET devices in closed state is the width of active area of the transistors. The devices of higher power capacity have lower impedance. This relation corresponds, in general, to the relation between the capacity and input impedance of audio speakers: more powerful speakers have lower input impedance. Required impedances can be easily calculated, and output-load matching can be reached even when MOSFET device is connected directly to the speaker without any impedance transformers. The low-pass filter between them, when they are used, has usually the same input and output impedances and doesn't affect the matching at all. This is one of the reasons why no matching circuits are used in audio applications, and the design of audio power amplifiers are often done to achieve its maximum efficiency.

In DSL applications the power amplifier should drive the POTS line with characteristic impedance $R_{\mathrm{l}}=100 \mathrm{Ohm}$. Such relatively high load resistance makes necessary to employ matching circuit in between the output of the amplifier and the line terminals. Equivalent electrical diagram of switching amplifier with matching circuit can be drawn as in Figure 4.4 below.

The condition of maximum power transfer for the switching amplifier with matching resistor is as follow.

$$
R_{\text {source }}+R_{\text {switch }}+R_{\text {match }}=R_{\text {load }}
$$




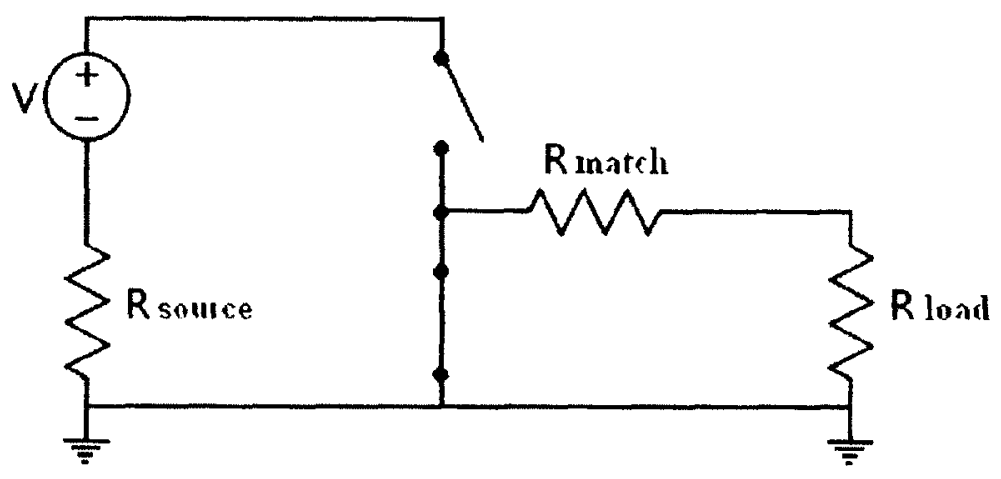

Figure 4.4 Switching Amplifier with Matching Resistor

The efficiency of the amplifier shall be:

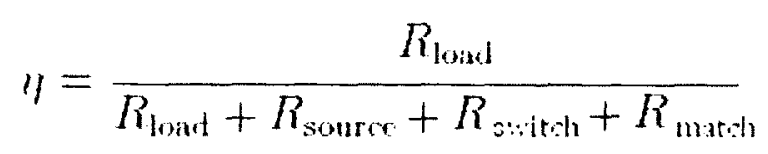

Being the part of power amplifier, the matching circuit will dissipate part of the signal power. The efficiency of the amplifier is not expected to be more that $50 \%$ as per Jacobi's law.

\subsection{Efficiency of Switching Power Amplifiers with Low- pass Filter}

One more consideration should be taken into account when the efficiency of switching amplifier is being discussed.

Square-wave signals at the input and output of switching amplifier are usually of the same amplitude. The amplifier produces the signal of higher power, i.e. it injects more current into load. 


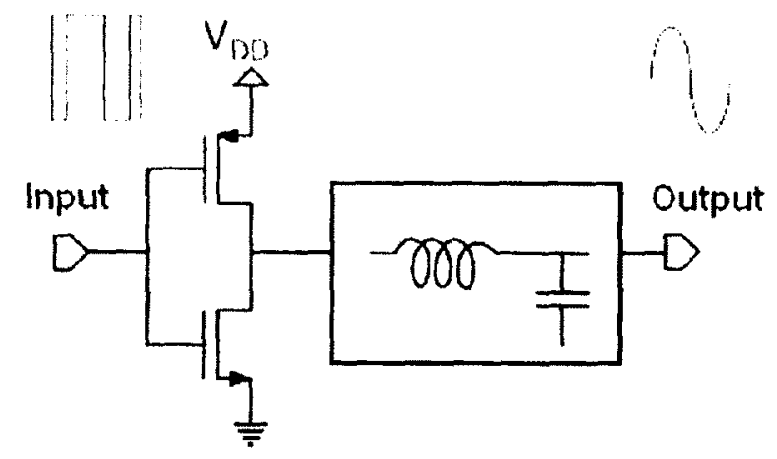

Figure 4.5 Switching Amplifier with Low-pass Filter

Square-wave signal at the input of the amplifier is the sum of odd integer harmonics. The signal can be represented using Fourier series:

$$
r(t)=\frac{t .4}{\pi} \sum_{n=1.3,5 \ldots} \frac{1}{n} \cdot \sin \left(\frac{n \pi t}{T / 2}\right)
$$

The first, or fundamental, harmonics has amplitude $4 \mathrm{~A} / \pi$. Its RMS value is smaller by a factor of $\overline{2}$.

At the same time the RMS value of square-wave signal with duty cycle $50 \%$ can be estimated using the following expression:

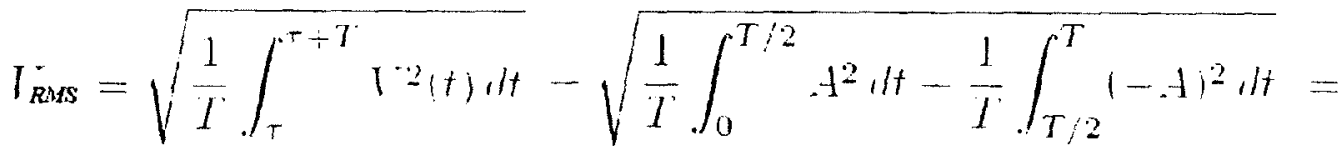

$$
\begin{aligned}
& -\sqrt{\frac{2 f^{2}}{1} \int_{0}^{T / 2} 11 /}-\sqrt{\frac{2.1^{2}}{T} \frac{1}{2}-A}
\end{aligned}
$$

The signal power is $P=V^{2} / R_{1}$., where $V$ is RMS amplitude of the signal, $R_{1}$, is load resistance. 
When switching amplifier is equipped with low-pass filter at the output that allows the fundamental tone only to pass to load terminal, the efficiency of the amplifier is as follow.

$$
\frac{P_{\text {Jumiamentul }}}{P_{\text {squareude }}}=\frac{\left(\frac{4 A}{\sqrt{2}}\right)^{2} / R L}{A^{2} / R L}=\frac{16}{2 \pi^{2}}=\frac{s}{\pi^{2}} \approx 81 \%
$$

This shows that the efficiency of switching amplifier with low-pass filter at the output is at best $81 \%$ regardless of the efficiency of the switches.

This conclusion is valid for square-wave symmetrical signal with $50 \%$ duty cycle. The efficiency of the switching amplifier degrades when square-wave signals of other duty cycle are applied to its input.

\subsection{Self-oscillating Power Amplifiers for ADSL Applications}

Self-Oscillating Power Amplifiers (SOPA) are often considered in technical literature as a solution for ADSL/VDSL line drivers. The SOPA for DSL application was initially proposed and comprehensively characterized by T. Piessens and M.

Steyaert in 2003 [3]. The solution allows a designer to build the line driver using standard digital CMOS technology. The authors developed the architecture that maintains high efficient amplification of the signals with high pulse-to-average ratio such as DMT signals. The block diagram of basic zero-order SOPA is shown in Figure 4.6 below. 


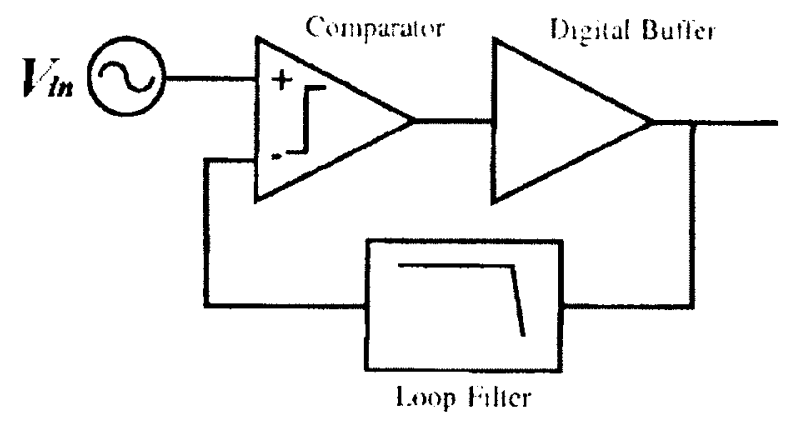

Figure 4.6 Basic Zero-order SOPA

Single SOPA building block consists of a comparator, digital multistage buffer and a loop filter. The function of digital buffer is to convert low-power square-wave signal into a high-power square-wave signal. From signal point of view, the buffer adds a parasitic delay only. The SOPA building blocks of higher order include integrator(s) as shown in Figure 4.7 below. $\mathrm{N}$-order SOPA has $\mathrm{N}$ integrators in a loop.

The topology of SOPA blocks is somewhat similar to the topology of $\Delta \Sigma$ modulators, but it is not the same. Asynchronous delta modulation is used in the SOPA. The amplifier is self-running block that is not clocked by external signal. The switching frequency is relatively low. As reported in [3] the mean value of the frequency is just 1.9 $\mathrm{MHz}$ for zero-order SOPA and $3.8 \mathrm{MHz}$ of third-order device.

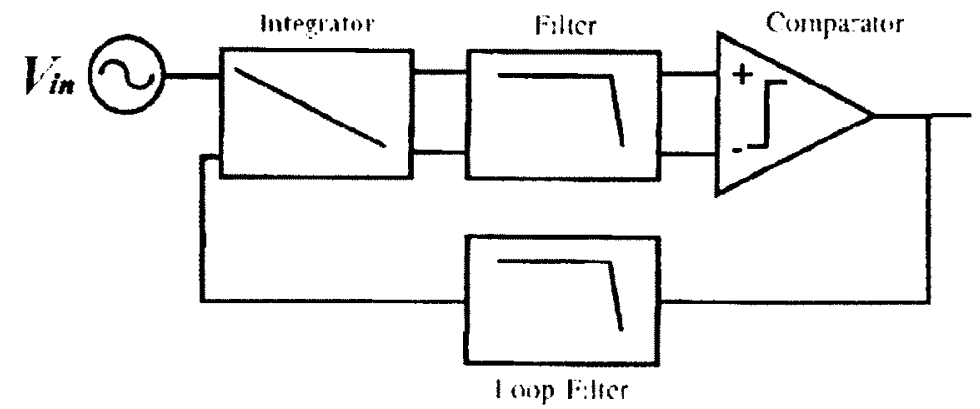

Figure 4.7 First-order SOPA 
The input signal of the amplifier is analog. As the output signal of digital buffer appears to be square-wave, the authors suggested using two coupled amplifiers as shown in Figure 4.8 below to reduce high-order harmonics at the output. Since the SOPAs are not clocked, the self-oscillation frequencies of the two asynchronous building blocks are pulled to each other. The mean switching frequency becomes a common-mode signal. Since a line transformer is used for coupling, the switching frequency is decoupled towards the line. Spurious-free dynamic range of the circuit in Figure 4.4 is $\mathrm{SFDR}=56 \mathrm{~dB}$.

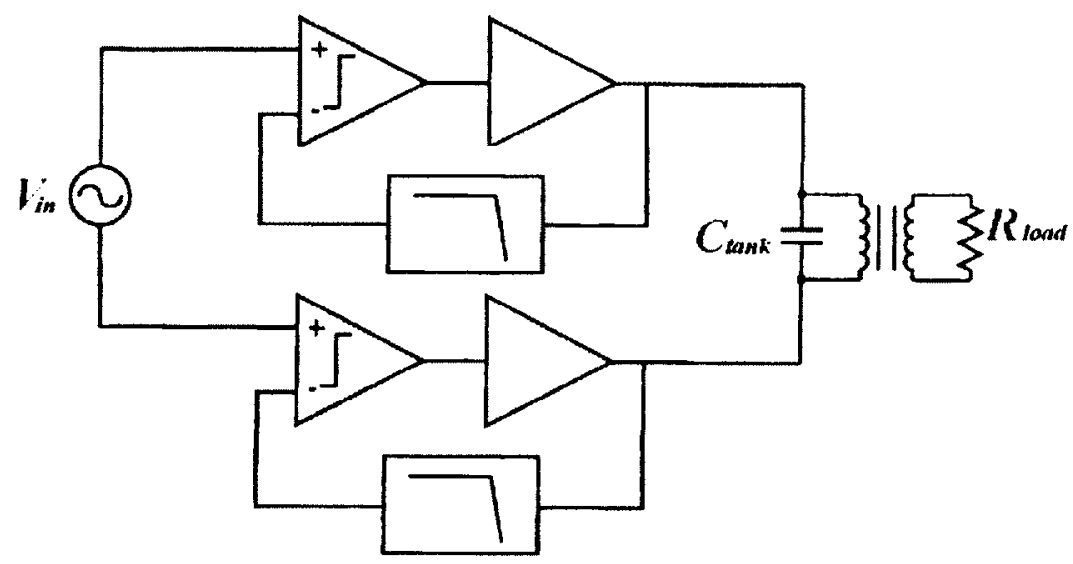

Figure 4.8 Dual SOPA Topology

The SOPA was designed and fabricated using standard $0.35 \mu \mathrm{m} 3.3 \mathrm{~V}$ CMOS technology. The zero-order SOPA line driver provides MTPR $=41 \mathrm{~dB}$ and bandwidth $\mathrm{BW}=800 \mathrm{kHz}$ that meets $\mathrm{G}$-lite requirements. Third-order SOPA provides MTPR=56 $\mathrm{dB}$ and bandwidth $\mathrm{BW}=8.6 \mathrm{MHz}$ that allows to use the device for VDSL applications. The efficiency of the amplifiers is $41 \%$ and $47 \%$ respectively. 


\subsection{Implementations of Switching Power Devices in}

\section{Low-voltage CMOS}

Advanced CMOS technologies are characterized by low voltage supply headroom. This issue poses serious problem for the design of efficient line driver for DMT signal, which have high PAPR value. The authors in [29] believe that a highly efficient ADSL line driver in low-voltage CMOS technology is a contradiction. As the solution, they proposed the SOPA that includes high-voltage output buffer.

The SOPA architecture is proved to be quite successful in digital sub-micron CMOS since it can drive efficiently the DMT signals with high PAPR value. However, as with any power amplifier, its efficiency and reliability drop significantly with decreasing voltage headroom. The authors believe that lowering the supply voltage results in increased current density to maintain constant output power. This, in turn, increases hot carrier generation and electro-migration, both of which affect the reliability of the driver. The large current also results in a drop of efficiency because of increased switching and conduction losses of the driver. Moreover, the large PAPR value causes the driver to put signals with high-voltage swing on the line. Since the output voltage swing of the driver is limited by its supply voltage, a transformer with high turns ratio should be used. However, this increases the return signal attenuation that limits the practical use of the line driver.

The proposed solution includes high-voltage buffer as the final stage of the multistage buffer. The high-voltage buffer is designed in standard submicron CMOS 
technology and doesn't require extra mask sets, which are usually used to fabricate high-voltage devices.

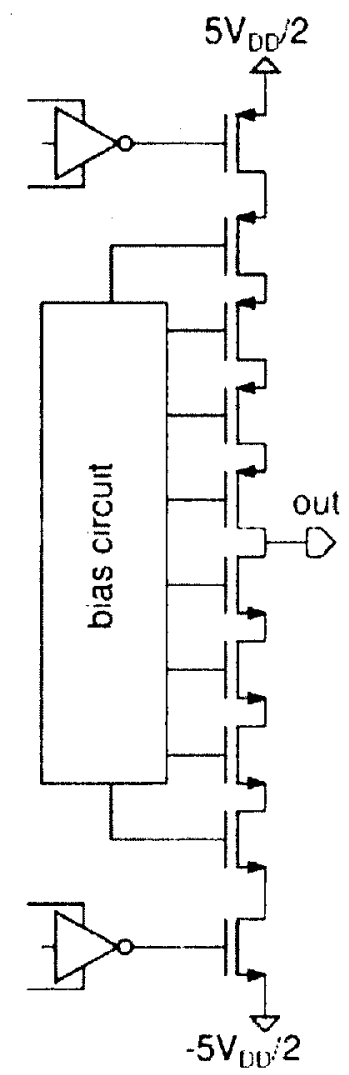

Figure 4.9 Block Schematic of High-voltage Driver

The driver is composed of 5 stacked transistors for pull-up circuit and other 5 transistors for pull-down circuit. The transistors are driven by dedicated bias circuit to keep the voltage across their terminals within the technology limit. If the nominal supply voltage is $\mathrm{V}_{\mathrm{DD}}$, the theoretical maximum supply voltage for the buffer can be as high as $5 \cdot V_{D D}$. This approach uses a symmetrical supply with two level-shift circuits for setting the offset voltages of the PMOS and NMOS transistors. The level-shifters are 
preceded by non-overlapping switching circuit to avoid short-circuit currents that can cause significant power dissipation at high voltages.

The driver was fabricated and tested with nominal power supply voltage $\mathrm{V}_{\mathrm{DD}}=$ 1.2 V. The high-voltage driver operated at supply voltage $\mathrm{V}=5.5 \mathrm{~V}$. When 512 -tone DMT signal is applied to the input of the SOPA with high-voltage driver, Missing Tone Power Ratio is MTPR $=58 \mathrm{~dB}$ with an average output power $20 \mathrm{dBm}$. These parameters allow using this device for ADSL2 applications. Measured efficiency of the driver is $42 \%$.

The stacked output stage is attractive from a cost point of view as it is implemented entirely with low-voltage devices, but its complexity makes it too slow for wide band systems (8 $\mathrm{MHz}$ and higher). [30] offered similar to the above SOPA architecture [29] with separate core low-voltage and driver high-voltage power supply, but developed new fast output stage. The output stage is implemented with NMOS devices only. This reduces by two the driven gate area compared to traditional NMOS/PMOS totem pole and so maximizes the switching frequency, minimizes switching losses and reduces the power consumption of the driver. Simulation showed that the bandwidth of the line driver is about $30 \mathrm{MHz}$, which is much higher than the bandwidth $\mathrm{BW}=2.2 \mathrm{MHz}$ of the line driver [29]. However, the price paid to get this improvement is significant. The MTPR reduced to $31 \mathrm{~dB}$ and the efficiency dropped to $23 \%$ only. 


\subsection{Switching Output Buffer with Wideband}

\section{Transformer}

Stacked output stage with voltage supply, that is higher than core logic voltage, allows getting required output power with lower drain current. Such exchange of higher current for higher voltage is not always necessary. Reliability degradation due to hotcarrier generation and electro-migration takes place because of high current density. However, current density can be maintained at reasonably low level in CMOS transistors, while the drain current through this transistor can be as high as necessary to obtain required output power. This is clear from the expression that defines the current through the transistors. The current depends, among other parameters, on the width of the active channel (or the width of gate terminal) that can be made as big as necessary. In triode (ohmic) mode, drain current $I_{l}$ ) of NMOS transistor is as follow:

$$
I_{D}=\mu_{n} C_{a r} \frac{\|^{\prime}}{L}\left(\left(V_{i s}-l_{t h}\right) l_{L S}-\frac{I_{D S}}{2}\right)
$$

where $V_{D S S}$ and $V_{G S}$ are voltage drops between drain and source and between gate and source terminals respectfully, $V_{l t}$ - threshold voltage, $\mu_{n}$ is charge-carrier mobility (electrons here), $C_{o x}$ is gate oxide capacitance per unit area, $L$ and $W$ are length and width of gate terminal. Similar expression exists for the PMOS transistor.

If current density can be maintained at reasonably low level by selecting proper width of the transistor channel, the standard PMOS/NMOS totem pole can be used to build the output buffer of the line driver. As the driver should manage relatively high current to provide required signal power at the line terminals, the internal resistance of 
the buffer (or switching circuit) should be low enough. This means that the line driver should include impedance transformer and matching circuit. The block diagram of CMOS buffer with associated circuitry is shown in Figure 4.10 below.

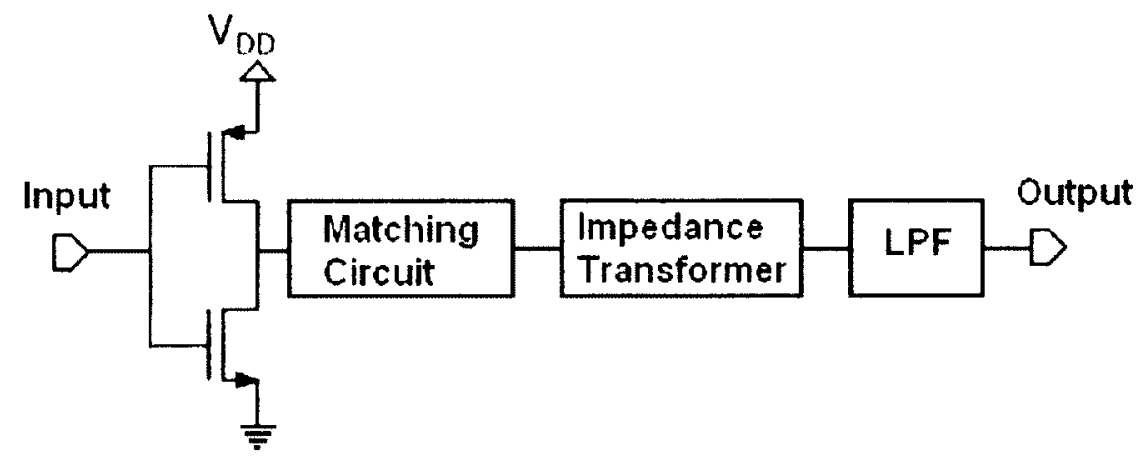

\section{Figure 4.10 Output CMOS Buffer with Impedance Transformer and LPF}

The necessity to have impedance transformer is defined by low-voltage power supply of CMOS buffer. To get the output power $\mathrm{P}_{\mathrm{OUT}}=20 \mathrm{dBm}$ at power supply $\mathrm{V}=$ $2.5 \mathrm{~V}$ the current through the transistor should be $\mathrm{I}=\mathrm{P}_{\text {out }} / \mathrm{V}=40 \mathrm{~mA}$. The output impedance of the circuit should be $\mathrm{R}=62.5 \mathrm{Ohm}$ minimum to provide required output power. As the CMOS buffer should work as the switch, its internal resistance in ON state should much smaller than $\mathrm{R}=62.5 \mathrm{Ohm}$. It is not realistic to assume that $\mathrm{CMOS}$ transistors behave as an ideal switch, but $\mathrm{R}_{\mathrm{ON}}=3-5 \mathrm{Ohm}$ resistance value would be acceptable. This will allow keeping the passive power dissipation in PMOS/NMOS totem pole low enough. Of course, the size of the transistors is going to be large to maintain the current density within technological range.

Push-pull circuit shown in Figure 4.10 above is known in technical literature as half H-bridge driver. CMOS transistors of the output buffer switch the load either to the 
voltage source or to the ground terminal. It is interesting to study full $\mathrm{H}$-bridge output buffer, which can be drawn as shown in Figure 4.11 below.

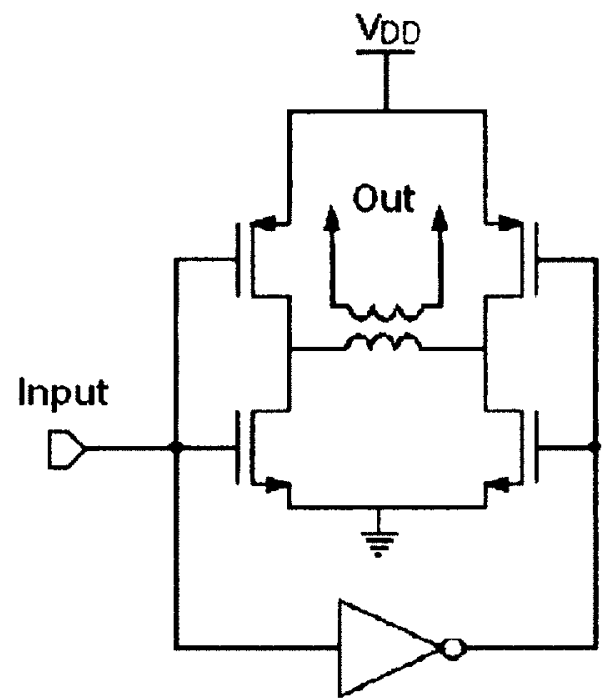

Figure 4.11 Full H-bridge Switching Buffer

Full H-bridge schematic solution might be promising in low-voltage power supply applications as it can be interpreted as current rather than voltage switch. However, its real implementation is challenging mostly because of the output load. The load impedance is transferred to the primary side of the transformer and appears to be inserted between the switching transistors, which are in $\mathrm{ON}$ mode and are conducting the current. The voltage drop on the load changes the voltage drops on the transistors and can change the mode of their operation. This might cause the degradation of the power efficiency of the switching stage.

Switching mode of the CMOS output buffer is associated with dynamic power dissipation. Two main processes contribute to this type of parasitic power consumption. 
CMOS circuits dissipate power by charging/discharging the various node/load capacitances (mostly gate and wire capacitance, but also drain and source capacitances) whenever they are switched. In one complete cycle of CMOS logic, current flows from $V_{D D}$ to the load capacitance $C_{1}$ to charge it and then flows from the charged load capacitance to ground during discharge stage. Therefore, in one complete charge/discharge cycle, a total charge $Q_{s w}=C_{L} V_{D D}$ is transferred from $V_{D D}$ to ground. Leakage current is the product of the charge Qsw multiplied by the switching frequency $f_{S w}$ :

$$
\mathbf{I}_{S w}=Q_{S w} \cdot f_{S w}
$$

The power dissipated by CMOS device due to the switching is:

$$
P=I_{S W} \cdot V_{D D}=C_{L} \cdot V_{D D}^{2} \cdot f_{S W}
$$

If the load capacitance is estimated on a node together with average switching frequency, the dynamic power dissipation at that node can be calculated effectively. As one can see, dynamic power dissipation due to the switching is higher with high node/load capacitances, which rise when the dimensions of the node/load gets larger. It is worth to note that it is beneficial to use low-voltage power supply, as the switching loss increases when $V_{D D}$ goes up.

The second process that contributes to the dynamic power dissipation is socalled short-circuit power dissipation. Since there is a finite rise/fall time for PMOS and NMOS devices, both transistors can be ON for a short moment of time during transition from one state to the other. Some current will flow from $V_{D D}$ directly to ground, hence 
creating a short circuit current. Short circuit power dissipation increases with rise and fall time of the transistors.

An additional mechanism of power consumption became significant in advances CMOS technologies as wires on chip became narrower, and the long wires became more resistive. CMOS gates at the end of those resistive wires see slow input transitions. During the middle of these transitions, both the NMOS and PMOS logic networks are partially conductive and current flows directly from $V_{D D}$ to $V_{S S}$. The power thus wasted is called crowbar power and similar to short-circuit power dissipation. Careful design, which avoids weakly driven long skinny wires, can ameliorate this effect, and crowbar power can be done substantially smaller than switching power.

\subsection{Conclusions}

This section begins with review of Class D power amplifiers that are well known for their high efficiency in audio applications. The main component of Class D power amplifiers is switching output stage, which dissipates very small amount of power.

The switching amplifiers were studied as the line drivers in DSL applications. Surprisingly, but the available publications report of relatively low, $50 \%$ or less, efficiency of switching output buffers. The authors believe that the degradation of the driver's efficiency takes place because of high PAPR value of the signal. They state that it is difficult to amplify such signal when voltage supply headroom is limited. This explanation is reasonable to some extent, although the losses due to the matching 
circuits, dynamic power dissipation and, potentially, low-pass filtering should be taken into account as well.

It seems strange, but no design solutions are published on the DSL line drivers that use $\Delta \Sigma$ modulator as data encoder for switching output buffer. The architecture of the line driver with $\Delta \Sigma$ modulator doesn't require traditional Class D power amplifier with analog input and analog output. The output of the $\Delta \Sigma$ modulator is single-bit data stream, or two-level digital like signal. When such encoder is employed, the power amplifier should just pump certain amount of energy to the output terminals without changing the shape of the signal.

Preliminary study reveals that generic push-pull NMOS/PMOS totem pole might be good enough as a switching line driver for DSL applications, however, the final decision should be made based on simulation results and following testing of real fabricated device in a lab.

It is not expected that the efficiency of the line driver will be as high as it is for audio Class D power amplifiers. Higher frequency bandwidth of DSL signals increases the power loss due to such things as matching and dynamic power dissipation that are not essential in audio applications. 


\section{ADSL Switching Line Driver with $\Delta \Sigma$}

\section{Modulator}

The design of the ADSL line driver that consists of the sigma-delta modulator followed by the switching amplifier is studied below. The architecture has been discussed in internet community, but no real technical solutions are proposed in professional publications.

The line driver is designed as a completely digital device. Such approach is innovative, in particular, when $\Delta \Sigma$ modulator is considered. Most $\Delta \Sigma$ modulators, especially, of $2^{\text {nd }}$-order and higher, are made as the analog devices as per known publications. It is very appealing to build the device using digital technique only as the digital design gets the advantages of modern advanced CMOS technologies. It also seems to be logical for transmission path as the input signal for the line driver is generated by IFFT signal processor, which is always implemented as digital device. Removing digital-to-analog and following analog-to-digital conversions will supposedly improve the performance of the device.

The line driver is composed of $\Delta \Sigma$ modulator, connected in series to the switching amplifier. This topology allows splitting the design for two relatively independent steps. The $\Delta \Sigma$ modulator is designed as RTL code in Verilog HDL that can be easily captured in schematic using well known tools from Altera, Xilinx, Synopsis and others available on the market. The switching amplifier is designed with Cadence 
design suite. The Cadence ADE appears to be the convenient instrument that allows integrating design entries made with different tools (HDL editors, schematic capture) and then simulating the overall design.

\section{1 $\Delta \Sigma$ Modulator Design}

The choice of HDL and the style of the design are defined mostly by the tools available for this work. Cadence Virtuoso Design Environment Rev. 5.10.41 includes Verilog Editor that is compatible with Verilog IEEE 1364-1995 Standard. The Verilog code, when compiled, allows creating schematic cellview that makes easy for a designer to integrate Verilog-based modules in Virtuoso Schematic Capture. Composite schematic can be further simulated in Analog Design Environment.

The Verilog code, when verified for the required functionality, can be used to synthesize the schematics using standard tools.

\subsubsection{The Topology of $\Delta \Sigma$ Modulator}

The switching amplifiers deal always with the signals of binary waveforms. The data is encoded in the signal using pulse-width modulation, pulse density modulation (sometimes referred to as pulse frequency modulation), sliding mode control (often called "self-oscillating modulation") or $\Delta \Sigma$ modulation. All these types of modulation are combined in the class of continuous-time processing.

$\Delta \Sigma$ modulation allows also discrete-time implementation, which supposes that the signal is represented by its values taken at some discrete moments of time. The $\Delta \Sigma$ modulator, which Simulink model is comprehensively investigated in Section 3.4 above, is the discrete device. Accordingly, the modulator designed for this work is the 
discrete device. The model of $\Delta \Sigma$ modulator, which is used to design the Verilog code of the device, is shown in Figure 5.1 below.

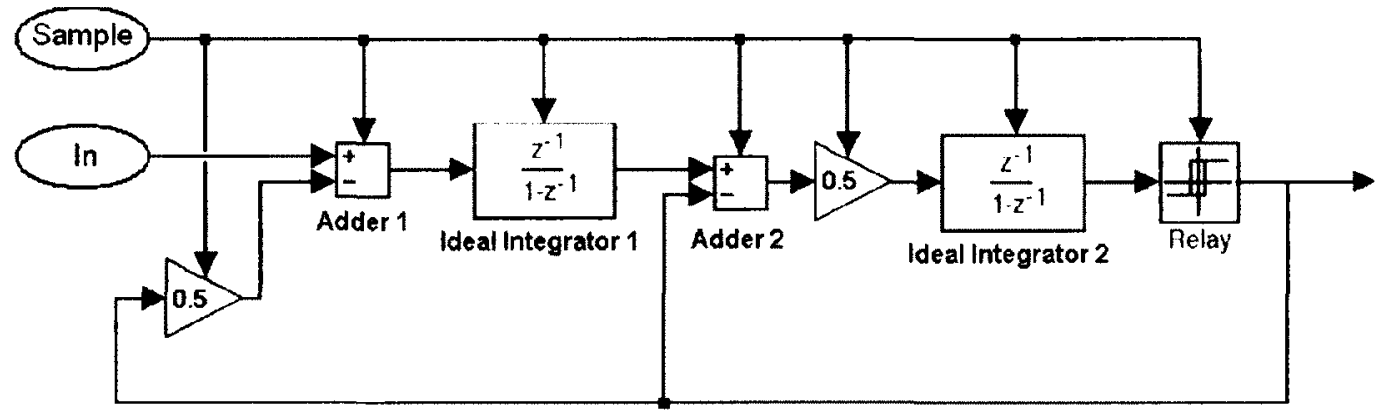

Figure 5.1 2nd Order Sigma-Delta Modulator

The simulations showed that the performance of the $\Delta \Sigma$ modulator is very sensitive to the value of its internal parameters. The parameters define the loopback gain of the device and the distribution of feed-forward gain from its input to the output. It is found that the best performance of the modulator takes place if the gain of each of its two stages is equal. Also for better performance the proportion of feed-forward signal and loopback signal at the input of adder modules should be equal for both modules in the model. The relation between the saturation level of the Ideal Integrators and signal amplitude of Relay module strongly affects the signal-to-noise ratio at the output of the $\Delta \Sigma$ modulator as well. Certainly, the optimal set of the model parameters can be justified theoretically, if it is not done already.

It is worth to note that the quantitative relations of the parameters of the $\Delta \Sigma$ modulator designed for this work in Verilog HDL completely correspond to their counterparts of the Simulink model. 
The discrete nature of the $\Delta \Sigma$ modulator defines its distinctive feature when the modulator is built as a pure digital device. The execution of the functional operation within each modulator's module should be completed in one sample/clock period or less, so that the signal change at the input of the module and corresponding signal change at the output take place within one clock period. This actually means that the signal propagation delay within each module of the $\Delta \Sigma$ modulator should be less than the clock period.

The feasibility of such requirement is defined by the CMOS technology used to fabricate the device. $0.18 \mu \mathrm{m}$ CMOS is supposed to be suitable. This technology allows designers to fabricate reasonable fast and relatively low-voltage devices, which might be no such expensive compared to more advanced silicon technologies. The detailed discussion of the relevant features of $0.18 \mu \mathrm{m}$ CMOS is provided below.

The signal propagation delay in the modules of the digital $\Delta \Sigma$ modulator is functionally similar to the slew rate of the operational amplifiers that are the parts of the legacy analog $\Delta \Sigma$ modulators. The slew rate strongly affects the performance of the analog $\Delta \Sigma$ modulators that are built with switched-capacitor integrators. Similarly, in digital modulators large propagation delays can completely destroy the signal transformation along its whole path from the input to the output of the device.

The constraint on the module's propagation delay is very critical for the Ideal Integrator module of the $\Delta \Sigma$ modulator. The modulator includes two Ideal Integrators, which define the order of the $\Delta \Sigma$ modulator and, accordingly, its performance. The functional block diagram of the integrator is shown in Figure 5.2 below. 


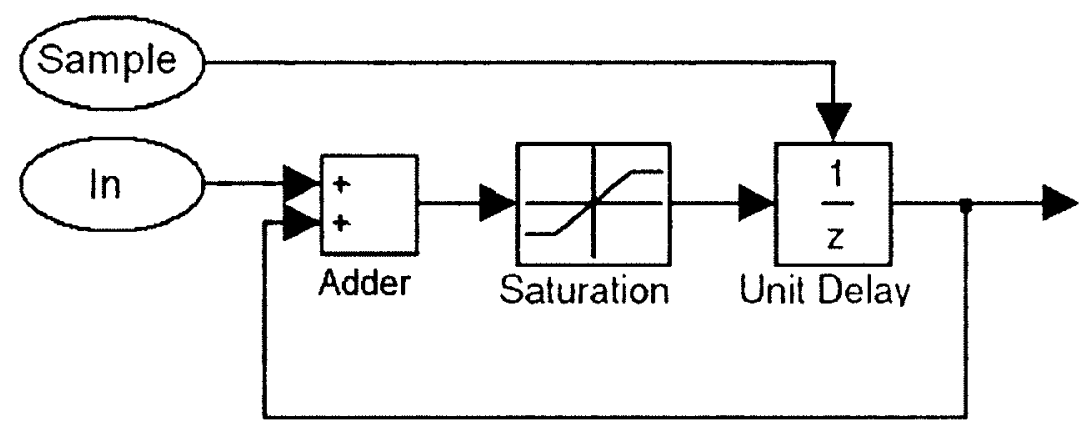

Figure 5.2 Functional Block Diagram of Ideal Integrator

The integrator operates properly when the overall delay of its Adder and Saturation blocks are less than one sample period. The Unit Delay block of the Ideal Integrator makes the total signal propagation delay of the integrator equal to one sample period. If this is the case, the integrator behaves as required. If the total signal propagation delay of the integrator is more than one sample period, the performance of the digital sigma-delta modulator can change in unpredictable manner.

The signal propagation delay of the modules composing the digital sigma-delta modulator is different. As the digital implementation of the operations performed by the modules are known, the signal propagation delay in the modules can be estimated in quite a reasonable manner. For this purpose the number of gates, which the signal should go through within each module, shall be determined. The delay of the gate for particular CMOS technology is usually known. It is specified in the libraries of standard cells developed for that technology. For $0.18 \mu \mathrm{m}$ CMOS the gate delay (2-input NAND. fanout $=2$, internal gates) is reported to be from 39 ps (Fujitsu 6-metal CS80A process, [311) to $43.6 \mathrm{ps}$ (6-layer interconnect process, Epson S1L70000 Series Gate Arrays, 
1321). Similar gate delays have been demonstrated by the ring oscillators built with TSMC $0.18 \mu \mathrm{m}$ 1P6M CMOS technology.

The value $t_{p}=45 p s$ shall be used below as the propagation delay of standard NAND gate built with $0.18 \mu \mathrm{m}$ CMOS technology. The propagation delay in each module of the $\Delta \Sigma$ modulator is estimated as the gate delay multiplied by the number of gates in the longest signal path in the module.

The amplifier with the gain $\mathrm{G}=0.5$ is actually the divider by two. This operation corresponds to shifting the whole digital word/code by one bit to the left. The implementation of the logical shift is simple: bit $\mathrm{N}$ of the input word/code is wired to bit $\mathrm{N}-1$ of the output word/code. The most significant bit of the output code is always equal to logical zero. The operation doesn't require any digital processing and implemented by proper signal wiring. The delay of such operation is insignificant and can be ignored at all.

The saturation operations in the Ideal Integrator and Relay modules require some processing. The processing algorithm can be represented by the schematics shown in Figure 5.3 below. The schematic is quite simple and allows estimating the contribution of the saturation blocks of the Ideal Integrator and Relay blocks in the signal propagation delay. 


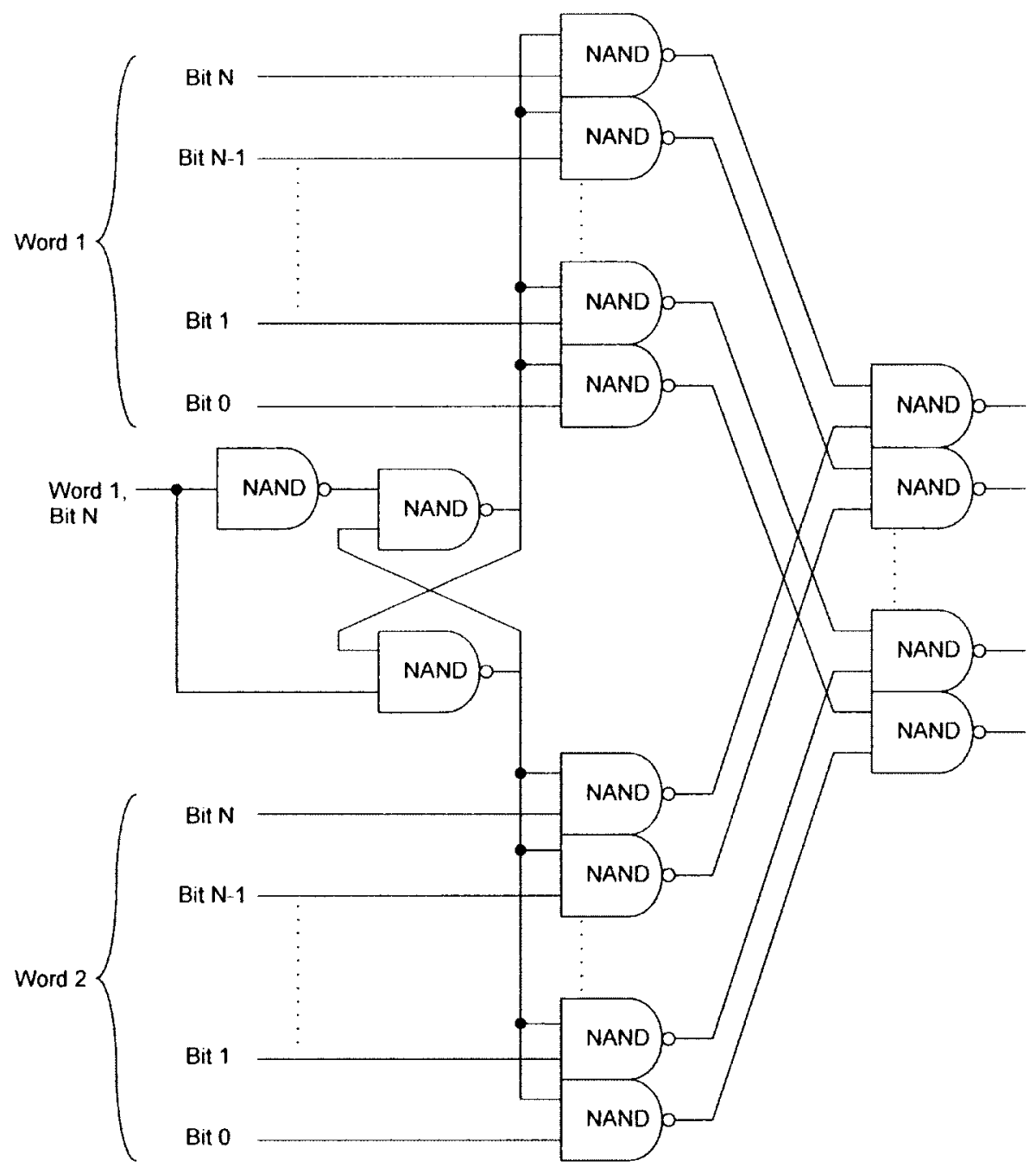

Figure 5.3 Saturation Schematics

The most critical path is made of the RS latch followed by two gates connected in series. The total number of gates that signal should pass through is four.

The adder blocks make the largest contribution in the signal propagation delay. Different architectures of the adder blocks are known. The ripple carry adder, being simple in concept, has a long circuit delay due to the many gates in the carry path from the least significant bit to the most significant bit. For a typical design, the longest delay 
path through an $n$-bit ripple carry adder is $2 n+2$ gates. Thus, for the 16 -bit adder the longest path that the signal goes through includes 34 gates. The carry look-ahead adder is the alternative solution. This adder has the architecture with reduced critical signal path at the price of more complex hardware. In 16-bit carry look-ahead adder the longest signal path includes 10 gates only [33].

The width of the signal word should be defined in order to estimate the propagation delay of the modules of the $\Delta \Sigma$ modulator. As specified in $|26|$, the maximal number of bits per sub-carrier supported by ADSL transmitter is $8 \leq \mathrm{Bit}_{\max } \leq 15$. This means that the signal level should be maintained by 16-bit data format as a minimum. Wider data format would be desirable to reduce the quantization error; however, this introduces the extra delays in the modulator's modules that might cause essential degradation of the modulator's performance.

As mentioned earlier, excessive delays in the digital modules of $\Delta \Sigma$ modulator cause unwanted SNR degradation at the output of the modulator and, as a result, the reduction of its dynamic range. The digital circuits with large delays are unable to react on the fast signal variations that might happen during the transitional time of digital device. As a result, signal distortion takes place. This effect is similar to the signal clipping in analog devices. The fast changing signals with high PAPR, such as DSL signals, are particularly vulnerable to such effect.

The $\Delta \Sigma$ modulator is designed for 16-bit signal width. The number of signal bits might be increased only if the performance of the ADSL line driver doesn't meet the relevant specifications. 
The modules' delays of the 16-bit $\Delta \Sigma$ modulator are given in Table 1 below.

Table $1 \Delta \Sigma$ Modulator's Modules Delay

\begin{tabular}{|l|l|l|}
\hline \multicolumn{1}{|c|}{ Module } & \multicolumn{1}{|c|}{$\begin{array}{c}\text { No of gates in the } \\
\text { critical path }\end{array}$} & Delay \\
\hline Adder 1 & 10 & $450 \mathrm{ps}$ \\
\hline Ideal integrator 1 & $10+4$ & $630 \mathrm{ps}$ \\
\hline Adder 2 & 10 & $450 \mathrm{ps}$ \\
\hline Divider by 2 & 1 & $45 \mathrm{ps}$ \\
\hline Ideal integrator 2 & $10+4$ & $630 \mathrm{ps}$ \\
\hline Relay & 4 & $180 \mathrm{ps}$ \\
\hline Divider by 2 & 1 & $45 \mathrm{ps}$ \\
\hline
\end{tabular}

The Simulink model of the $\Delta \Sigma$ modulator has been studied with the sample period $T_{S}=7.8125 \mathrm{~ns}$ (sample frequency $f_{S}=128 \mathrm{MHz}$ ). The sample period is 10 times larger than the maximum delay observed in the modules of the $\Delta \Sigma$ modulator provided it is designed with $0.18 \mu \mathrm{m}$ CMOS. This allows to build the modulator, which performance is not degraded due to the technology restrictions.

\subsubsection{Verilog Modules}

The $\Delta \Sigma$ modulator is designed as a set of modules of the same hierarchy. Cadence Verilog compiler generates the schematic symbol upon successful code compilation of each module. The Verilog module symbols can be integrated with the schematics of the switching amplifier for further simulation. 
Unsigned arithmetic is used to simplify the schematic implementation of Verilog modules during synthesis. The unsigned arithmetic is possible when the DC offset is added to the signal propagating in the $\Delta \Sigma$ modulator.

The signal at the input of the modulator has the offset equal to the half of its total range. This offset level corresponds to zero voltage level for an analog signal propagating inside of the $\Delta \Sigma$ modulator without DC offset. Switching signal polarity in unsigned arithmetic with half-range signal offset corresponds to changing the most significant bit from one logic level to the other one. The determination of the signal polarity in HDL conditional operands can be done easily by checking the value of the most significant bit of signal word only. This definitely reduces the corresponding electronics

Similarly, minimum and maximum of the signal at the output of ideal integrators inside the $\Delta \Sigma$ modulator are limited to the levels that are defined by two most significant bits of the signal words, $11000 . .00$ and $01000 . .00$. This makes necessary to check these two bits only in HDL conditional operands and so to reduce the signal delays in corresponding hardware modules due to less electronics. The signal extremes at the output of the integrators are equal to $\pm 50 \%$ of total signal range.

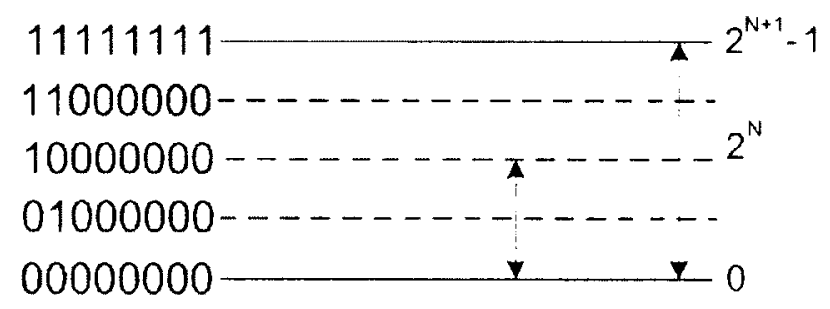

Figure 5.4 Signal Code Space 
Such selection of signal levels inside the $\Delta \Sigma$ modulator guarantees that no signal distortion takes place. Signal maximum at the output of the modulator's adders can be as large as $2^{\mathrm{N}+1}-1$, but never exceeds this value. Signal minimum can reach $00 . .00$ at the output of the adders, but never switches to $11 \ldots 11$ code. At the same time, the signal saturation due to over-ranged signal levels is prevented. The code space is used efficiently.

The schematic of the $\Delta \Sigma$ modulator with the component of the test bench is given in Figure 5.5 below.

Verilog modules, which compose the $\Delta \Sigma$ modulator, and their associated schematic symbols are provided in Appendix B.

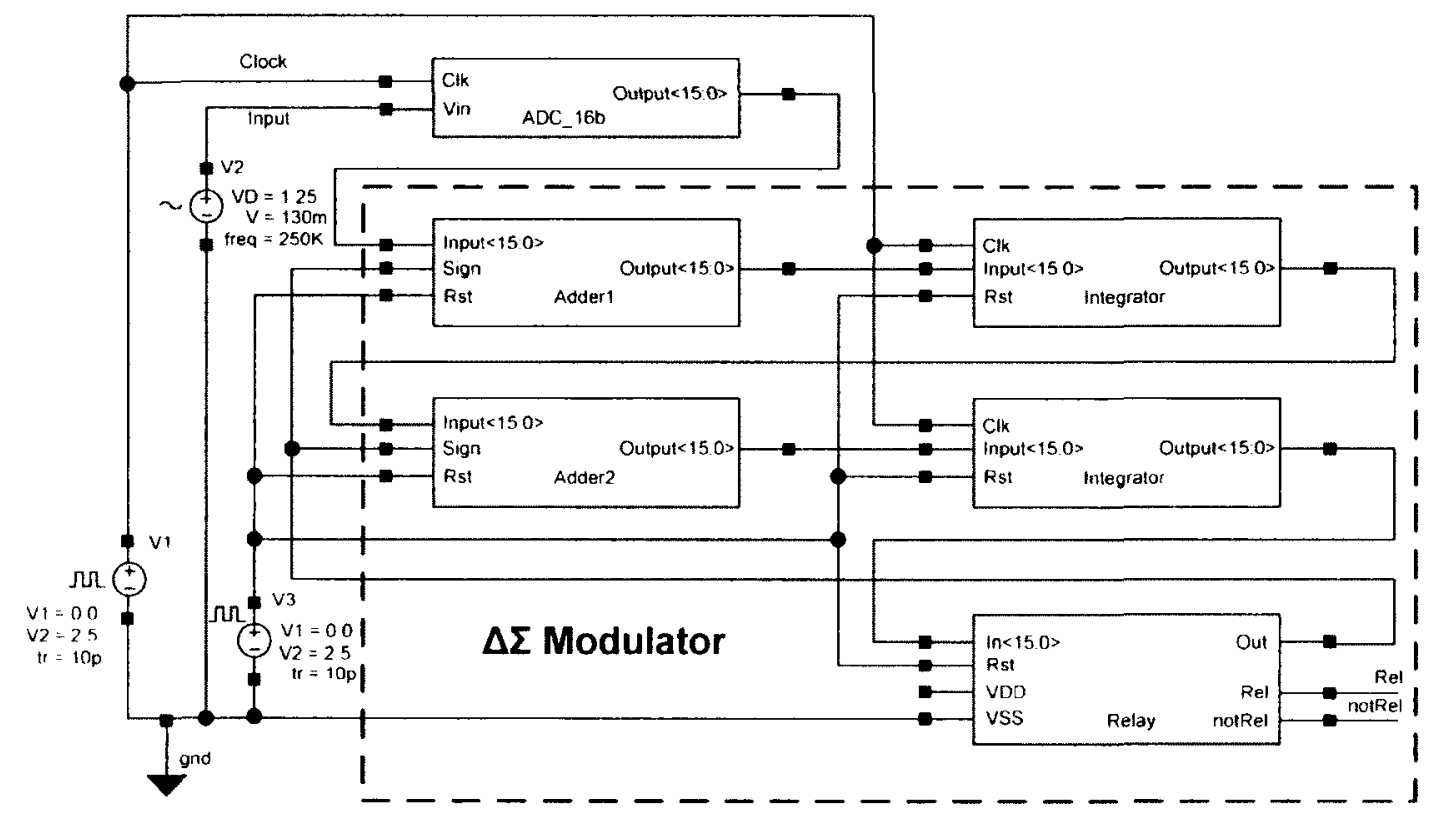

Figure 5.5 $\Delta \Sigma$ Modulator Schematic 


\subsection{Switching Amplifier Design}

As discussed in Section 4, the switching power amplifier made of generic CMOS inverters is one of the most effective and easy solutions for ADSL line driver. Both half-bridge or full-bridge configurations of the line driver are studied and compared below.

The main parameter of the inverter is the size of the CMOS devices of totempole transistor pair. The size is defined mostly by the technology used to fabricate the circuitry.

The selection of the CMOS technology for the switching power amplifier is somewhat intuitive. The following considerations are taken into account:

- The circuitry should be able to operate with the clock frequency up to 260 $\mathrm{MHz}$;

- The standard voltage supply should be high enough to avoid too high current in the output NMOS/PMOS totem pole;

- Technological current density limit should be high enough to withstand the required current;

- The transistor geometry should be small enough to avoid the clock frequency reduction due to too large parasitic capacitances.

The most reasonable choice for the switching power amplifier is the CMOS with $0.18 \mu \mathrm{m}$ minimum geometry size. The advantages of $0.18 \mu \mathrm{m}$ CMOS as related to the switching power amplifier for ADSL applications are as follows: 
- Maximum clock frequency could be as high as $3 \mathrm{GHz}[34 \mid$ for the standard transistors size used in digital applications;

- Recommended power supply voltage covers $1.8-3.3 \mathrm{~V}$ range that gives some freedom for a designer;

- The power supply voltage is high enough that allows to maintain low current in the transistors;

- Maximum allowed current density ( $1 \mathrm{~mA} / \mu \mathrm{m}$ for metal lines and $0.28 \mathrm{~mA} /$ $\mu \mathrm{m}$ for vias/contacts) is high enough to withstand the expected currents with minimal possible transistor size.

The actual maximum toggle frequency of the inverter in the switching power amplifier is expected to be much lower than $3 \mathrm{GHz}$ because of relatively large transistor size. Parasitic capacitances of the large transistors are larger than of the small ones. This causes the toggle frequency to drop. The actual maximal frequency of the inverter with the transistors of some specific size can be estimated in the circuit simulation.

The supply voltage headroom that is possible in $0.18 \mu \mathrm{m}$ CMOS is not high enough to deliver $100 \mathrm{~mW}$ power into $\mathrm{R}=100 \mathrm{Ohm}$ load, even when maximum power supply voltage $\mathrm{V}_{\mathrm{DD}}=3.3 \mathrm{~V}$ is used. The voltage/impedance transformer can be inserted in front of the load terminals as shown for the half-bridge amplifier architecture in Figure 5.6 below. 


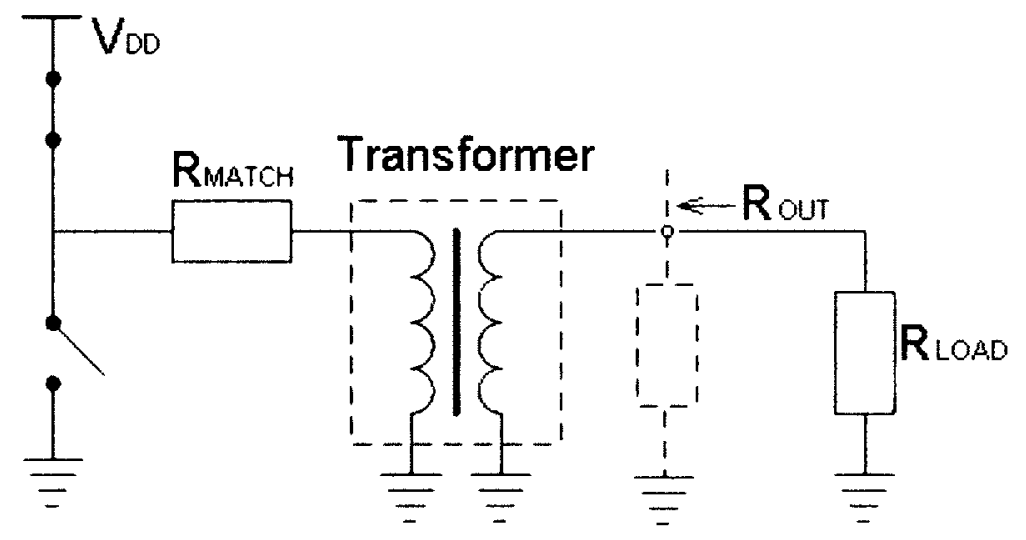

Figure 5.6 Functional Block Diagram of Switching Amplifier with Connected Load

In the block diagram $\mathrm{R}_{\mathrm{MATCH}}$ includes $\mathrm{ON}$ resistance of the switch and internal resistance of the power supply.

As per Jacobi's law, the output impedance $R_{\mathcal{O U T}}$ should be equal to the load impedance $\mathrm{R}_{\mathrm{LOAD}}$ to obtain maximum power on $\mathrm{R}_{\mathrm{LOAD}}$ from the source.

Without the transformer $\mathrm{R}_{\mathrm{MATCH}}$ should be equal to $\mathrm{R}_{\mathrm{IOAD}}=100 \mathrm{Ohm}$. Maximum current that can be developed in the load is $I_{M A X}=V_{S S} /\left(R_{I .(A I)}+R_{M A T C H}\right)$. When $\mathrm{V}_{D D}=3.3 \mathrm{~V}$ is used, $\mathrm{I}_{\mathrm{MAX}}=16.5 \mathrm{~mA}$. Accordingly the power in the load is $27.225 \mathrm{~mW}$, which is lower then $\mathrm{W}=100 \mathrm{~mW}$ required for $\mathrm{ADSL}$ line driver at $\mathrm{CO}$ location.

When the transformer is used, the requirement for the supply voltage headroom can be relaxed. With transformer ratio of secondary coil turns $N_{S}$ to the primary coil turns $N_{1} N=N_{S}: N_{P}=3: 1$, the load, when looked from the primary side of the transformer, has resistance $\mathrm{R}_{\mathrm{IOAD}}=100 / \mathrm{N}^{2}=11 \mathrm{Ohm}$ only. Accordingly, $\mathrm{R}_{\text {MATCH }}$ 
resistor is reduced to this value also. With $\mathrm{V}_{\mathrm{DD}}$ as low as $2.5 \mathrm{~V}, \mathrm{I}_{\mathrm{MAX}}$ is as high as 114 $\mathrm{mA}$, and the power in the load is $140 \mathrm{~mW}$.

As noted above $R_{\text {MATCH }}$ includes the internal resistance $R_{O N}$ of the CMOS transistor, either PMOS or NMOS, in open state. $R_{O N}$ is fixed and depends on the size of the transistor. The transistors of the switching amplifier are expected to be of relatively large size in order to get low internal resistance and high-power output signal. The final stage of the switching amplifier is preceded by two preliminary amplification stages with the transistors of smaller sizes. The transistors of the preliminary stages develop the signal to the certain power level and thus allow maintaining the rise and fall times of the pulses at the gates of the last amplifier stage short enough. The transistor sizes of first two stages are not optimized to get the minimum delay because the delay doesn't affect the overall performance of the line driver. The schematic of the switching power amplifier of half $\mathrm{H}$-bridge architecture is given in Figure 5.7 below.

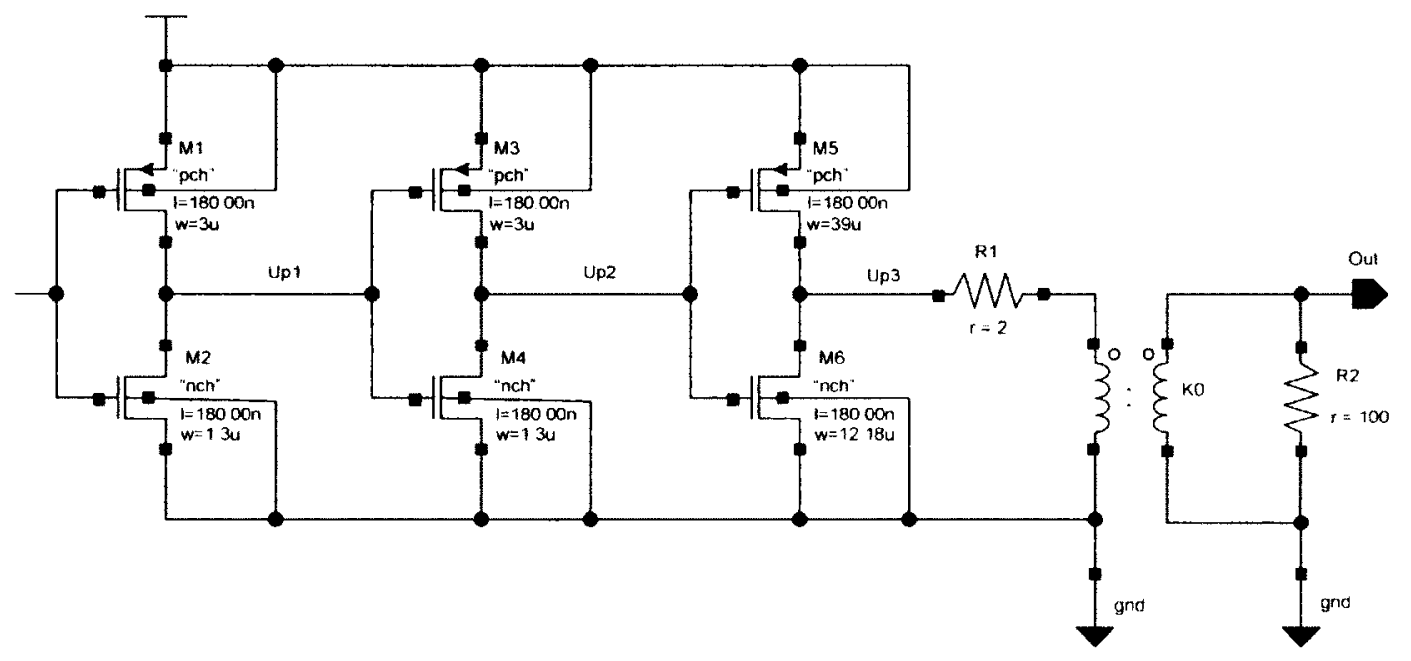

Figure 5.7 Half H-bridge Switching Amplifier Schematic 
The simulations of switching power amplifier revealed that the signal output power is quite sensitive to the matching conditions. There are three parameters in the schematic above that affect the matching, namely, transistor's internal impedance $R_{O N}$, matching resistance $R_{M A T C H}$ and transformer ratio $N$. $R_{O N}$ value depends mostly on the dimensions of the transistors, but it is also influenced by the latter two parameters. Indeed, the transistor impedance in open state can be roughly estimated as $R_{O N}=$ $\mathrm{V}_{\mathrm{DS}} / \mathrm{I}_{\mathrm{D}}$, where $\mathrm{V}_{\mathrm{DS}}$ is the voltage drop between the source and drain terminals of the switching transistor, $I_{D}$ is the current flowing through it. The current through the transistors depends on $\mathrm{V}_{\mathrm{DS}}$ voltage drop between drain and source terminals. The voltage is defined, first of all, by the power supply voltage. Also, the $\mathrm{V}_{\mathrm{DS}}$ value in the circuit in Figure 5.6 above depends on the voltage drop on $\mathrm{R}_{\mathrm{MATCH}}$ and $\mathrm{R}_{\mathrm{IOAD}}$ resistors.

$$
V_{D S}=V_{D D}-I_{D}\left(R_{M A T C H}+R_{L O A D} / N^{2}\right)
$$

As a result, it is practically impossible to make the preliminary estimations of the transistors dimensions and the values of the passive components around them. The circuit simulation is more effective way to determine the parameters of its components. This allows getting the transistors of minimal possible size while having maximum possible current through them.

It is worth to note here that the matching resistor helps to reduce the fluctuations of output signal due to the variations of the power supply voltage. When the voltage goes up, the current through the NMOS/PMOS transistors increases. This causes increasing the voltage drop on the matching resistor that reduces $\mathrm{V}_{\mathrm{DS}}$ voltage and brings I) current down. 
The simulations are done to determine the best matching conditions with matching resistors $\mathrm{R}_{\mathrm{MATCH}}=0,2$ and $3 \mathrm{Ohms}$ for the circuit that provides the signal of no less than $100 \mathrm{~mW}$ peak power at the line terminals. The results are summarized in Table 2 below.

Table 2 Half H-bridge Switching Amplifier

\begin{tabular}{|c|c|c|c|c|}
\hline R $\begin{array}{c}\text { PMATC } \\
{[\text { Ohm }]}\end{array}$ & Size, $[\mu \mathrm{m}]$ & Size,$[\mu \mathrm{m}]$ & Ratio & PouT, \\
\hline 0 & $\mathrm{~L}=0.180$ & $\mathrm{~L}=0.180$ & $41: 10$ & 104.5 \\
& $\mathrm{~W}=780$ & $\mathrm{~W}=250$ & & \\
\hline 2 & $\mathrm{~L}=0.180$ & $\mathrm{~L}=0.180$ & $48: 10$ & 100.8 \\
& $\mathrm{~W}=1260$ & $\mathrm{~W}=392$ & & \\
\hline 3 & $\mathrm{~L}=0.180$ & $\mathrm{~L}=0.180$ & $50: 10$ & 100.1 \\
& $\mathrm{~W}=2340$ & $\mathrm{~W}=672$ & & \\
\hline
\end{tabular}

The relative sizes of the PMOS and NMOS transistors are adjusted in order to get the same values of $R_{O N}$ internal resistances of NMOS and PMOS transistors in the open state. This equalizes the fall and rise times of the pulses propagating in the switching amplifier.

The rise and fall times of the pulses in between the stages and at the output of the amplifier in Figure 5.7 with $\mathrm{R}_{\mathrm{MATCH}}=2 \mathrm{Ohm}$ don't exceed $1.2 \mathrm{~ns}$. This means that the amplifier can be used with the switching signal of up to $400 \mathrm{MHz}$. 


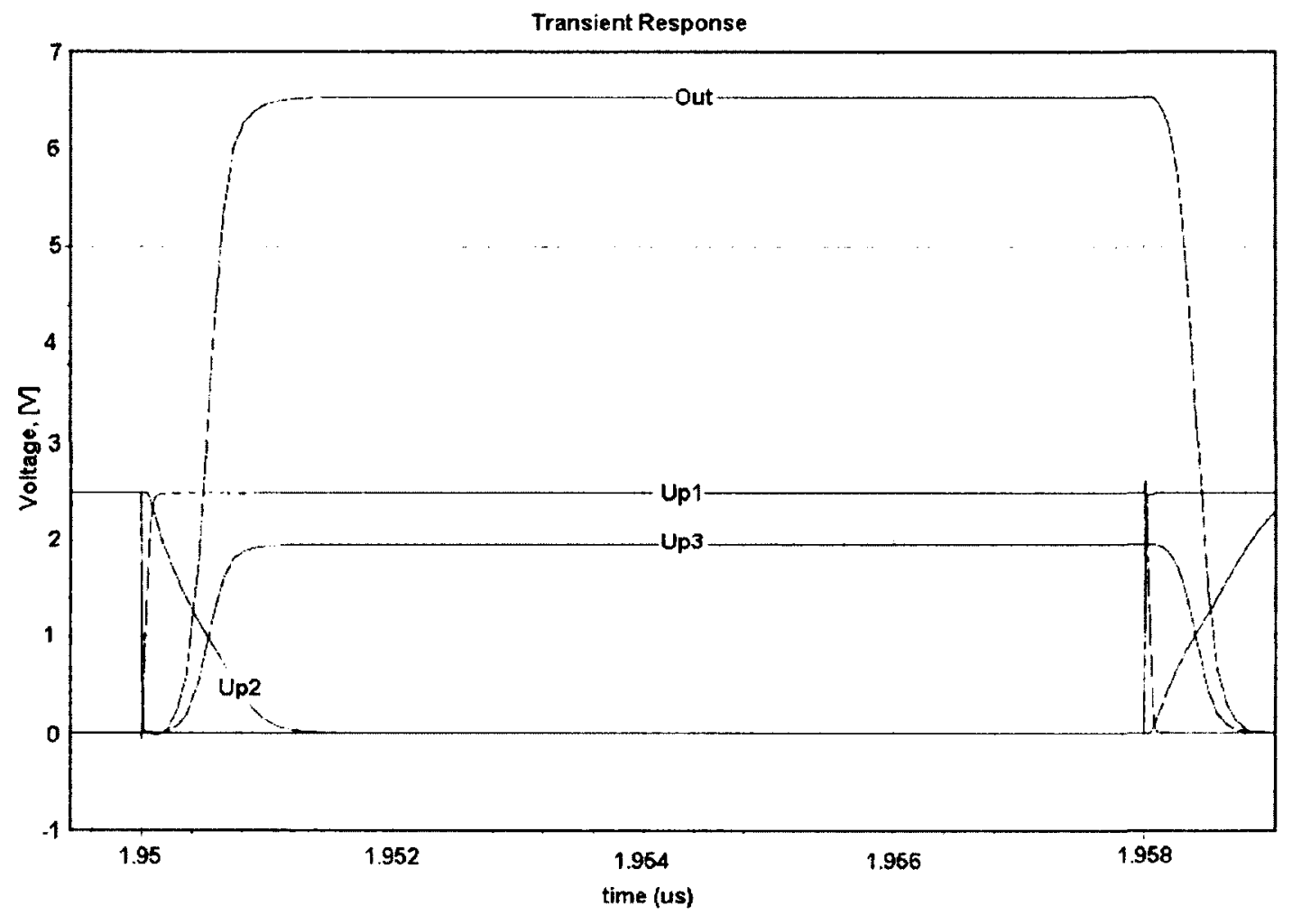

Figure 5.8 Half H-bridge Amplifier Pulses Shape

The power level of the signal is estimated as $P_{S I G}=A^{2} / 4 R_{1 . O A D}$, where $A$ is the signal voltage swing at the $R_{I . O A D}$ resistor terminals $\left(R_{L O A D}=R 2=100 \mathrm{Ohm}\right.$ in Figure 5.7 above).

The simulation shows that the output signal of the circuit in Figure 5.7 has the DC component. Its power is of the same level as the power of the signal itself. It is clear that the DC component doesn't carry any data and should be filtered out before being delivered to the line terminals.

The power efficiency of the circuits with different $R_{\text {MATCH }}$ values is estimated as the ratio of the signal power to the overall power supply consumption. The power consumption is downscaled by the duty factor of the signal, which is assumed to be 
$50 \%$ for the purpose of this exercise. The power efficiency figures of the circuit in Figure 5.7 above for different values of the matching resistors and, accordingly, for different transistors dimensions and transformer ratios are given in Table 3 below.

Table 3 Half H-bridge Switching Amplifier

\begin{tabular}{|c|c|c|c|}
\hline $\begin{array}{c}\mathrm{R}_{\mathrm{MATCH}}, \\
{[\mathrm{Ohm}]}\end{array}$ & $\begin{array}{c}\text { Pout, } \\
{[\mathrm{mW}]}\end{array}$ & $\begin{array}{c}\text { Power Efficiency, } \\
{[\%]}\end{array}$ & $\begin{array}{c}\text { Current Density, NMOS, } \\
{[\mathrm{mA} / \mu \mathrm{m}]}\end{array}$ \\
\hline 0 & 104.5 & 31.5 & 1.06 \\
\hline 2 & 100.8 & 26 & 0.78 \\
\hline 3 & 100.1 & 25 & 0.47 \\
\hline
\end{tabular}

The current in the switching transistors vary from $265 \mathrm{~mA}$ at $\mathrm{R}_{\mathrm{MATCH}}=0 \mathrm{Ohm}$ through $316 \mathrm{~mA}$ at $\mathrm{R}_{\mathrm{MATCH}}=3 \mathrm{Ohm}$. Drain-to-source voltage drop is less than $\mathrm{I} \mathrm{V}$ for all three cases. As one can see from Table 2 above, the size of transistors drastically increases with higher $\mathrm{R}_{\text {MATCH }}$ values. This reduces the current density in the switching transistors to the acceptable level (less than $1 \mathrm{~mA} / \mu \mathrm{m}$ ). However, it is very difficult, if possible at all, to build on the silicon a transistor of $2-3 \mathrm{~mm}$ width with predicted performance. That is why the half-H-bridge architecture of the switching amplifier can be used with matching resistor $\mathrm{R}_{\mathrm{MATCH}}=1-2$ Ohm only.

The efficiency level is expected to be half of maximum $50 \%$ level because of the DC component of the signal. However, the simulated efficiency is higher than $25 \%$ 
theoretical threshold. Such discrepancy can be the result of inaccurate estimation of the output signal peak power.

The discussion above is applicable to the half $\mathrm{H}$-bridge architecture of the switching amplifier. The full H-bridge amplifier architecture can be easily built as it is actually the extension of half $\mathrm{H}$-bridge architecture, but for differential signal. The schematic of the differential switching amplifier is shown in Figure 5.9 below.

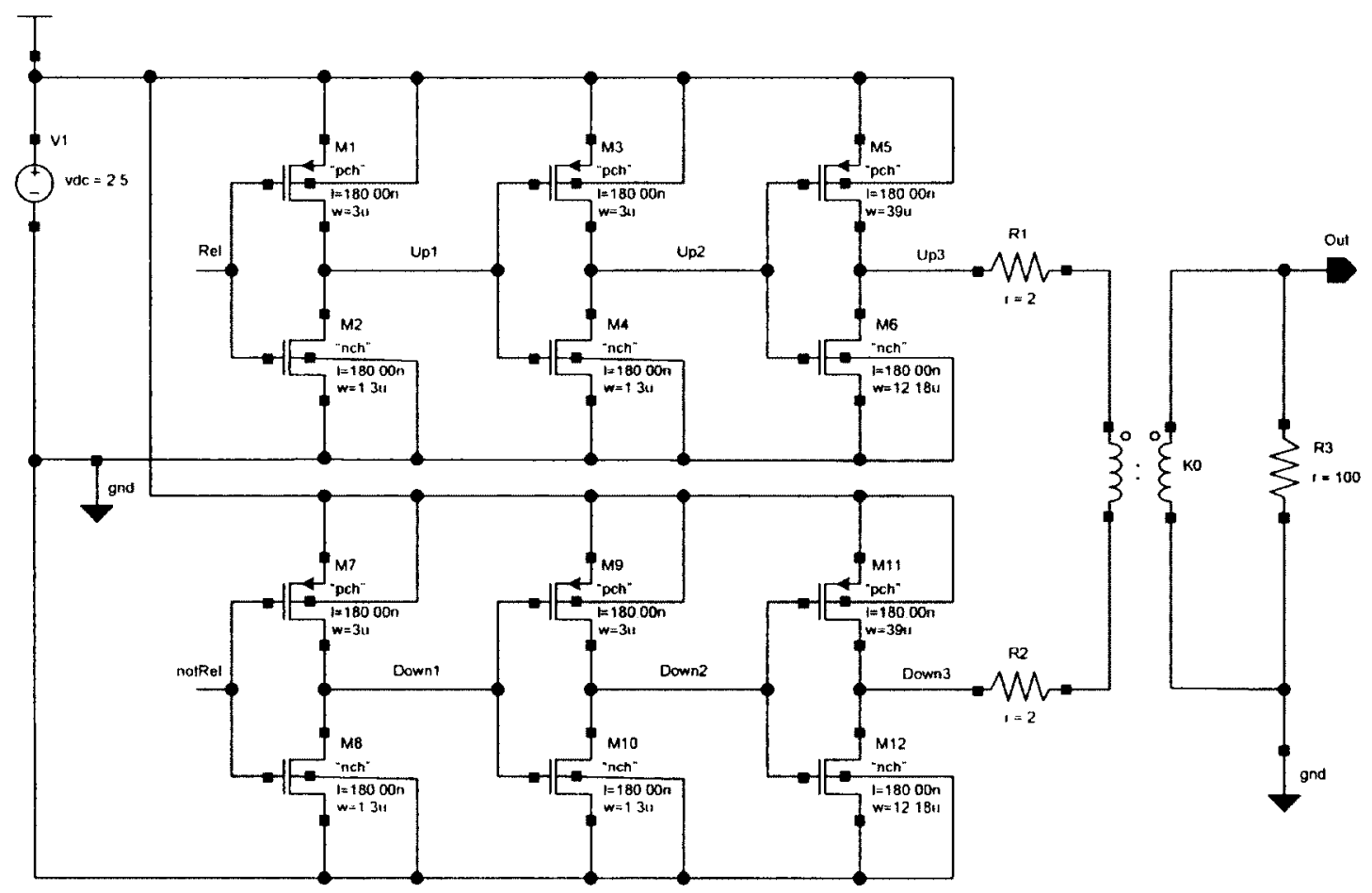

Figure 5.9 Full H-bridge Switching Amplifier

The circuit of full H-bridge switching amplifier includes the matching circuitry, which is adjusted during the simulation in order to get the maximum power in the load resistor. The transistor dimensions are set to minimum possible for the signal power 100 $\mathrm{mW}$. The transistors appeared to be less than half of the transistors size used in the half 
H-bridge architecture of the amplifier. The transformer ratio is changed significantly also to accommodate the new matching conditions. The matching is different not only because of different internal impedance of the switching transistors due to their smaller size, but also due to the fact that two transistors, NMOS and PMOS, are open at each moment of time, so the impedance of two of them connected in series defines the matching conditions.

The full H-bridge switching amplifier has been simulated with $\mathrm{R}_{\text {MATCH }}=0,2$ and $4 \mathrm{Ohm}$. The simulation results are summarized in Table 4 and Table 5 below.

Table 4 Full H-bridge Switching Amplifier

\begin{tabular}{|c|c|c|c|c|}
\hline $\begin{array}{c}\mathrm{R}_{\text {MATCH, }} \\
{[\text { Ohm] }}\end{array}$ & PMOS Transistor & NMOS Transistor & Transformer & Pout, \\
& Size, $[\mu \mathrm{m}]$ & Ratio & & \\
\hline 0 & $\mathrm{~L}=0.180$ & $\mathrm{LW}]$ & \\
& $\mathrm{W}=312$ & $\mathrm{~W}=100$ & & 101.8 \\
\hline 2 & $\mathrm{~L}=0.18$ & $\mathrm{~L}=0.180$ & $23: 10$ & 102 \\
& $\mathrm{~W}=396$ & $\mathrm{~W}=123.2$ & & \\
\hline 4 & $\mathrm{~L}=0.180$ & $\mathrm{~L}=0.180$ & $25: 10$ & 108.7 \\
& $\mathrm{~W}=663$ & $\mathrm{~W}=190.4$ & & \\
\hline
\end{tabular}

The circuits develop symmetrical signal. The DC component of it is negligibly small. The efficiency of full H-bridge switching amplifier is much better compared to its half $\mathrm{H}$-bridge counterpart. For the same level of output signal power the current 
amplitude through the switching transistors is about 4 times less. It is $67 \mathrm{~mA}$ for $\mathrm{R}_{\text {MATCH }}$ $=0 \mathrm{Ohm}$, and it increases to $82 \mathrm{~mA}$ for $\mathrm{R}_{\mathrm{MATCH}}=4 \mathrm{Ohm}$. As noted above, the transistors are much smaller in size; however, they are still quite large. The current density is below the technology limit $(1 \mathrm{~mA} / \mu \mathrm{m})$ for all three cases.

Table 5 Full H-bridge Switching Amplifier

\begin{tabular}{|c|c|c|c|}
\hline $\begin{array}{c}\mathrm{R}_{\text {MATCH, }} \\
\lceil\mathrm{Ohm}]\end{array}$ & $\begin{array}{c}\text { Pout, } \\
{[\mathrm{mW}]}\end{array}$ & $\begin{array}{c}\text { Power Efficiency, } \\
{[\%]}\end{array}$ & $\begin{array}{c}\text { Current Density, NMOS, } \\
{[\mathrm{mA} / \mu \mathrm{m}]}\end{array}$ \\
\hline 0 & 101.8 & 61 & 0.67 \\
\hline 2 & 102 & 56 & 0.60 \\
\hline 4 & 108.7 & 54 & 0.43 \\
\hline
\end{tabular}

The power efficiency of the full $\mathrm{H}$-bridge amplifiers is higher than $50 \%$ theoretical threshold. Such discrepancy can be the result of inaccurate estimation of the output signal peak power.

The relative sizes of the PMOS and NMOS transistors are adjusted in order to get the same values of $R_{O N}$ internal resistances of NMOS and PMOS transistors in the open state. This equalizes the fall and rise times of the pulses propagating in the switching amplifier. In addition to this, the DC component of the output signal is minimized. 
The rise and fall times of the pulses in between the stages and the output of the amplifier don't exceed 0.8 ns. This means that the amplifier can be used with the switching signal of up to $600 \mathrm{MHz}$.

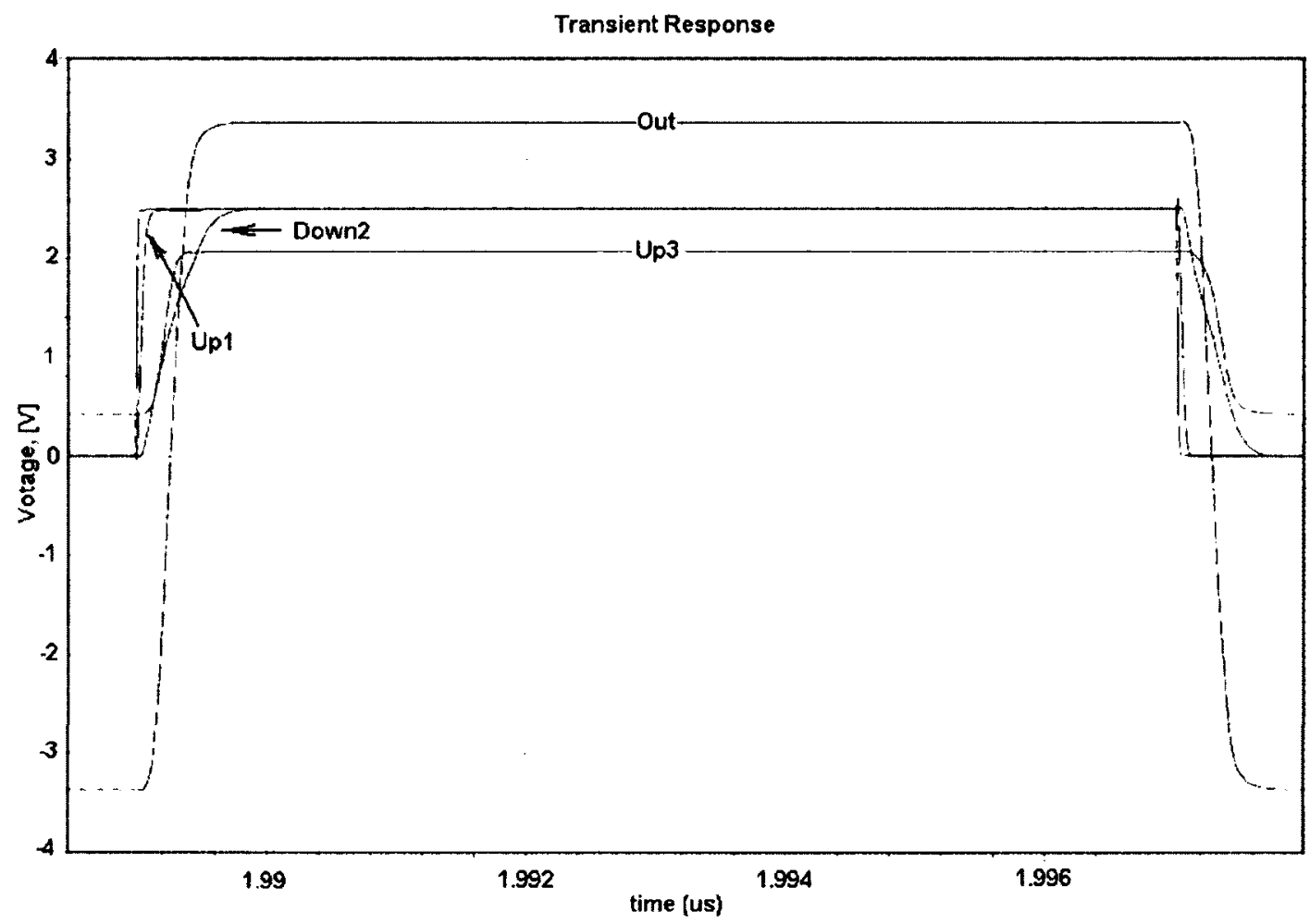

Figure 5.10 Full H-bridge Switching Amplifier Pulses Shape

The analysis of the simulation results shows the following advantages of the full $\mathrm{H}$-bridge switching amplifier compared to the half $\mathrm{H}$-bridge architecture of it.

- $\quad$ Power efficiency of the full H-bridge switching amplifier is higher

- No DC component is in the signal

- The current density is within the technology limits 
- The overall silicon area of the device per output power unit is significantly smaller

This proves that the full H-bridge switching amplifier is the potential solution for the ADSL line driver.

\subsection{System Verification}

\subsubsection{Test Benches and Tests Overview}

As noted above, the line driver is designed as completely digital device that consists of two main functional blocks, namely, the $2^{\text {nd }}$ order $\Delta \Sigma$ modulator followed by the switching power amplifier. The $\Delta \Sigma$ modulator is built as the set of Verilog modules. When they are compiled, the Cadence Virtuoso Design Suit creates the schematic symbols for each Verilog module. This allows incorporating the Verilog modules in the Virtuoso Schematic Capture tool.

The functionality of the line driver has been comprehensively simulated in Cadence Analog Design Environment with Spectra and SpectraVerilog simulators. Appropriate test benches are designed to apply appropriate stimulus signals and to capture the output signal for further analysis.

The following tests are done to characterize the performance of the ADSL line driver:

- The evaluation of Dynamic Range of output signal of the line driver;

- The evaluation of Missing Tone Power Ratio at the output of the line driver. 
The DR value at the output of the line driver defines the maximum number of bits that can be used to encode the signal at each sub-carrier of DMT signal employed in ADSL system. The DR evaluation is performed with single-tone sinusoidal signal applied at the input of the line driver.

The MTPR evaluation allows validating the performance of the line driver when multi-tone signal is applied at the input of the driver. The MTPR requirements for ADSL signal are provided in ITU recommendation [26]. The MTPR varies from $44 \mathrm{~dB}$ for 64-QAM to $65 \mathrm{~dB}$ for 256-QAM signals.

\subsubsection{The Test Bench and Verification of the $\Delta \Sigma$ Modulator}

16-bit digital signal is applied at the input of the $\Delta \Sigma$ modulator.

Cadence Virtuoso Schematic Capture has the built-in libraries of the components that were used to develop the test bench for the simulation of the $\Delta \Sigma$ modulator. In addition to the existing components, 16-bit Analog-to-Digital Converter (ADC) and Digital-to-Analog Converter (DAC) are designed. The ADC is necessary for the transformation of the analog signal of sine-wave generator to the digital data stream compatible with the input signal format of digital $\triangle \Sigma$ modulator. The DAC's are used in the modulator's test bench to monitor the signals generated inside the $\Delta \Sigma$ modulator.

The VerilogA HDL code of the ADC and DAC components is developed based on the designs of the similar components available in the Virtuoso libraries. The schematic symbols of newly designed components are generated by the Virtuoso Design Suite upon successful compilation of the corresponding VerilogA HDL code. New 
ADC and DAC are capable to manage 16-bit digital signals and the analog signals in 0 $-2.5 \mathrm{~V}$ amplitude range.

VerilogA code of ADC and DAC components used in the test bench of the line driver and their associated symbols are provided in Appendix $\mathrm{C}$ below.

The test bench for the characterization of the $\Delta \Sigma$ modulator is shown in Figure 5.11 below. The $\Delta \Sigma$ modulator itself is composed of Adder 1, Adder2, Relay and two Integrator components. The rest components belong to the Test bench. They provide the input signals to and capture the output signals from the modulator.

The stimulus signal for the SNR and dynamic range measurements is produced by the sine-wave generator, which analog output is transformed to the 16-bit digital data stream by the following ADC.

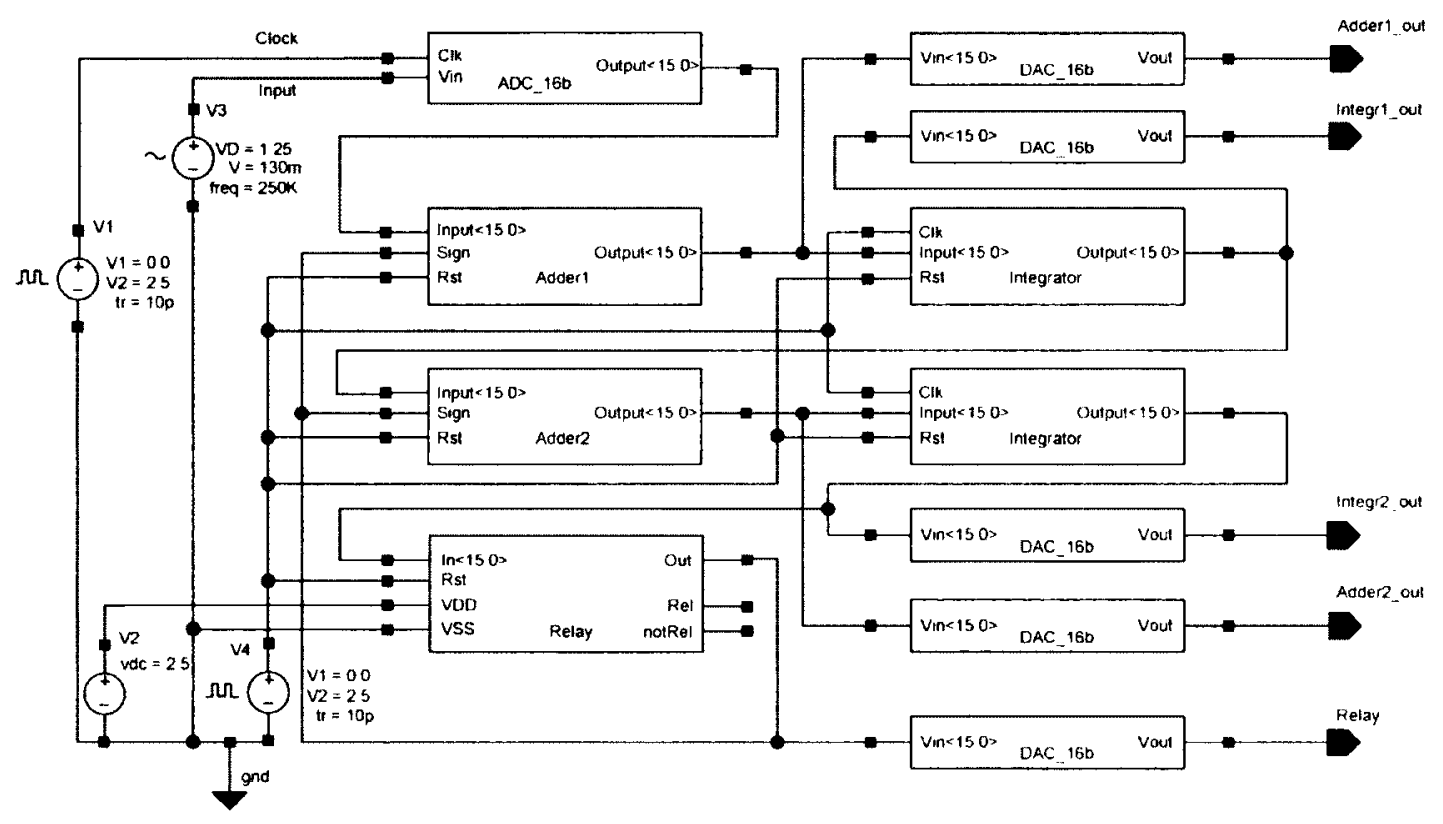

Figure 5.11 Sigma-Delta Modulator Test Bench 
Pulse generator $\mathrm{f}=128 \mathrm{MHz}$ provides clocking for the $\Delta \Sigma$ modulator and acts as a sample generator for the SNR and DR evaluations. The selection of the clock frequency is explained in Section 3.3 above.

The schematic includes multiple DAC modules to monitor the signals at the output of the internal modules. They are helpful in the process of the $\Delta \Sigma$ modulator design and troubleshooting. The screenshots of internal signals are shown in Figure 5.12 below.

Transient Recomes
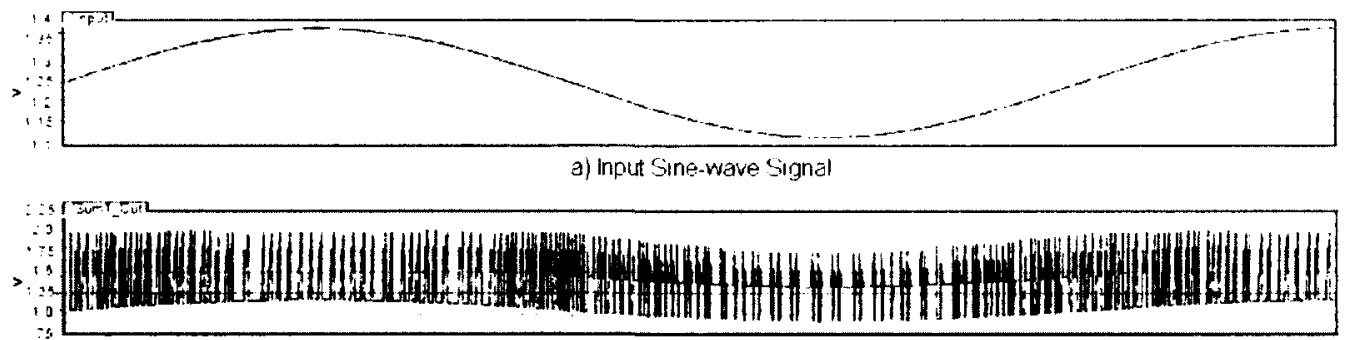

b) Output of Adder 1

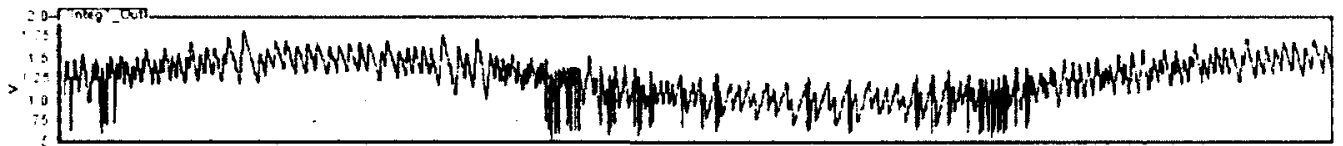

c) Cutput of ideal Integartor 1

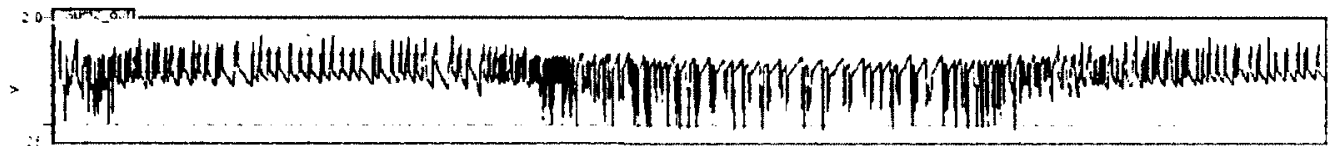

d) Cutput of Adder?

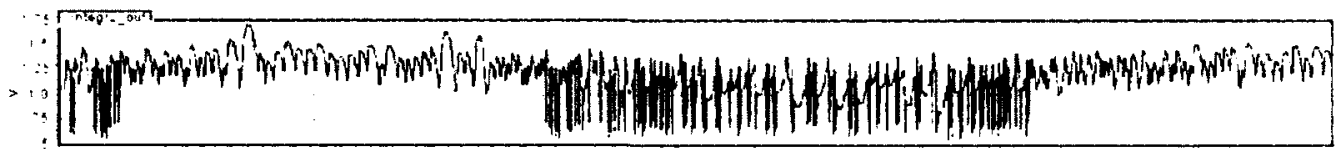

o) Gutput of integrator 2

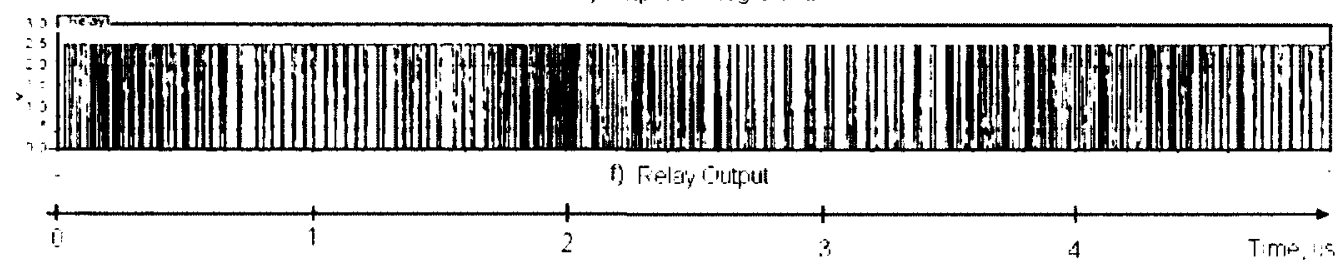

Figure $5.12 \Delta \Sigma$ Modulator's Internal Signals 
The output signal of the $\Delta \Sigma$ modulator is captured as CSV file and processed using the Matlab script that is similar to the one used to evaluate the Simulink model of the modulator.

The script calculates the Power Spectrum Density of the signal and the histograms of the signal amplitudes at the selected test points of the $\Delta \Sigma$ modulator. The typical example of the histograms at the output of the $1^{\text {st }}$ and $2^{\text {nd }}$ integrators is given in Figure 5.13 below. The histograms changes from Gaussian-like shape for the "noisy" analog signal at the input to a sharper shape that corresponds to the signal, which amplitude becomes close to the voltages of the digital signal domain.
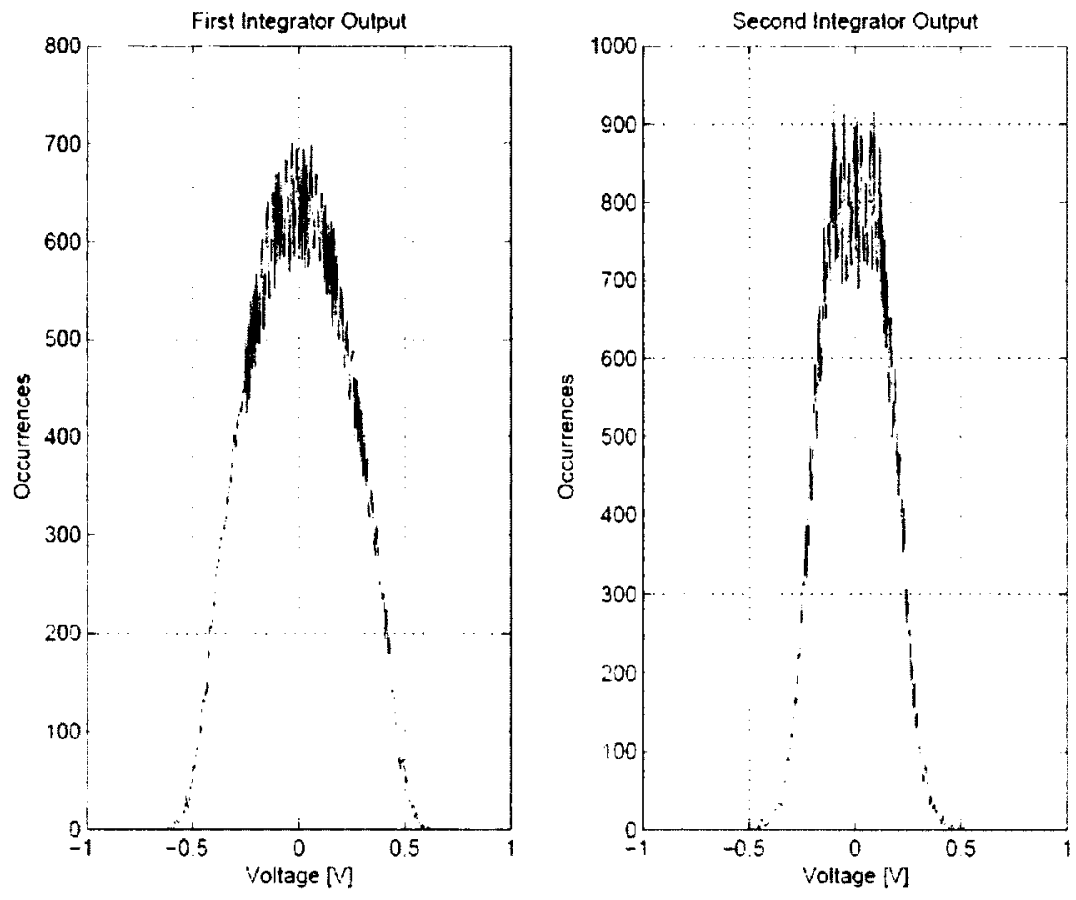

Figure 5.13 Signals Histograms of the $\Delta \Sigma$ Modulator 
The results of the SNR measurements are given in Figure 5.14 and Figure 5.15 below. The simulation is done for $f_{\mathrm{IN}}=250 \mathrm{kHz}$. The processing bandwidth is $\mathrm{BW}=$ $500 \mathrm{kHz}$.

It is worth to note that the circuitry doesn't include any low-pass filters; however, no high-order harmonics are observed in the signal at the output of the $\Delta \Sigma$ modulator. As per the definition of the Dynamic Range done in Section 3.3 the DR of the $\Delta \Sigma$ modulator in Figure 5.11 is at least $85.8 \mathrm{~dB}$.

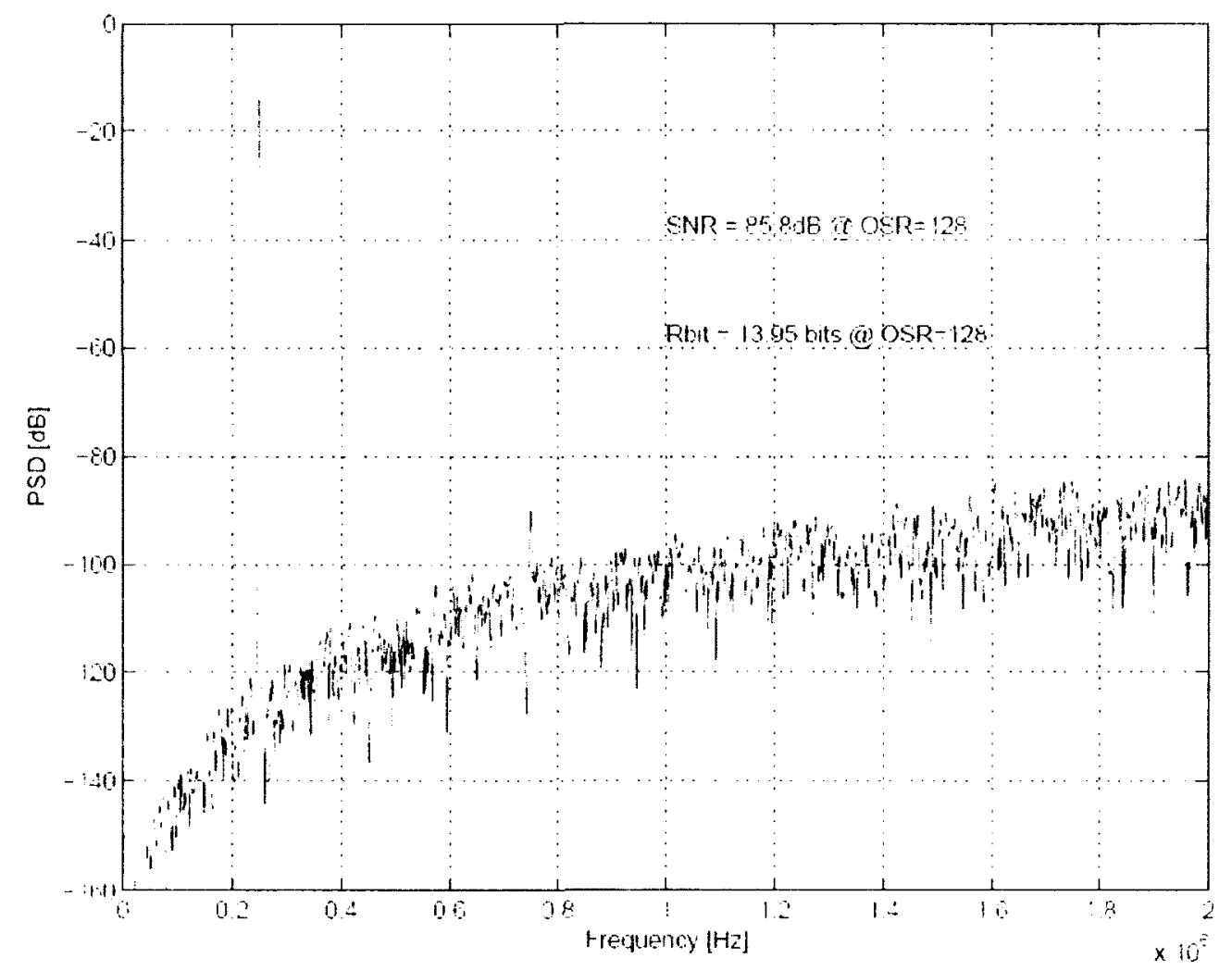

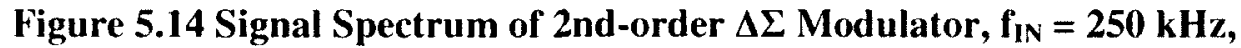
$\mathrm{BW}=500 \mathrm{kHz}$ 


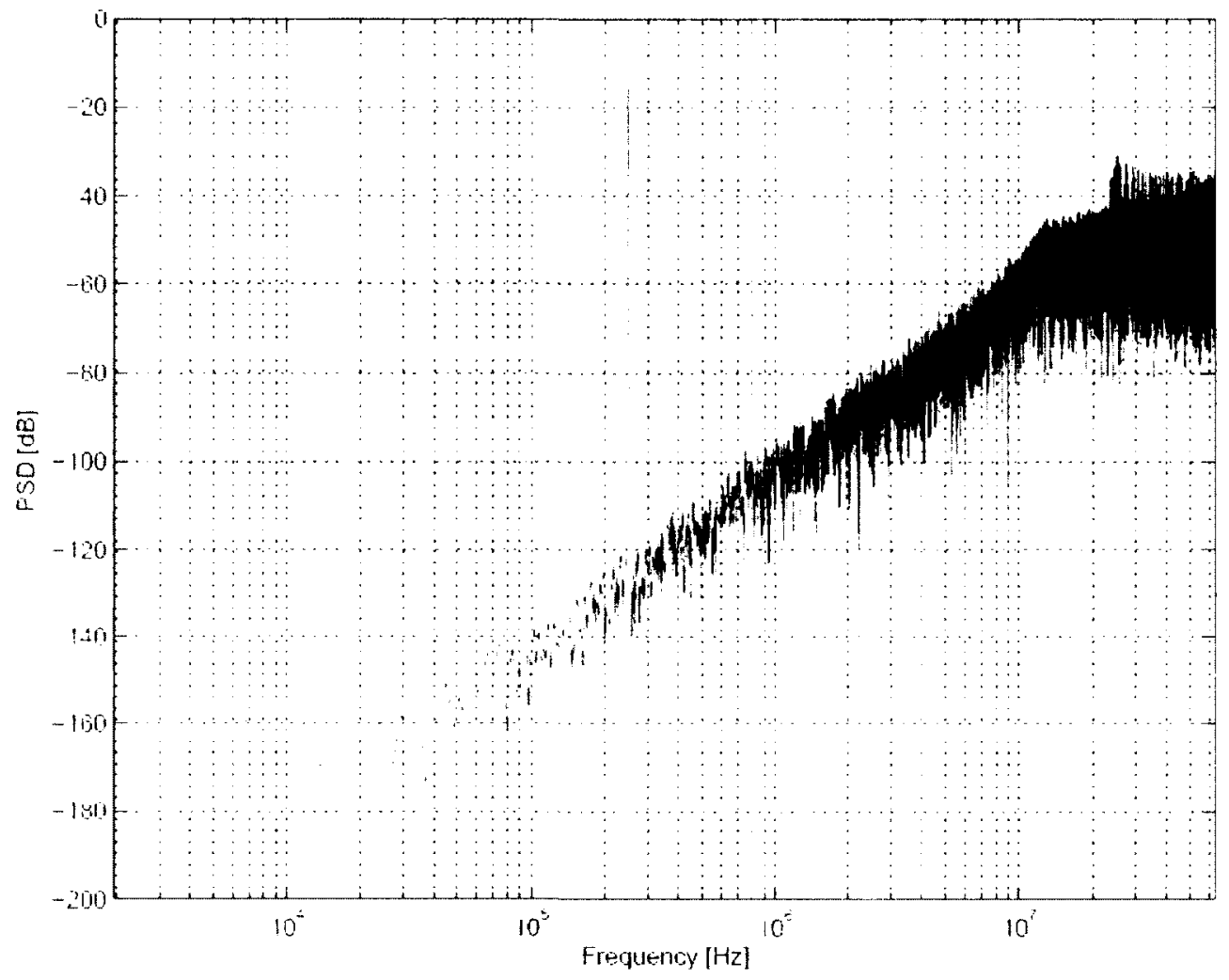

Figure 5.15 Signal Spectrum of 2nd-order $\Delta \Sigma$ Modulator, $f_{\mathrm{IN}}=250 \mathrm{kHz}$, $\mathrm{BW}=500 \mathrm{kHz}$

The MTPR evaluation is done with vpwlf component as the signal source instead of the sine-wave generator. The $v p w l f$ component includes the ascii file with the record of the signal that is generated by Simulink during the simulation of the ADSL modulator model [6]. The recorded signal represents the instantiation of the 256-QAM DMT signal, but with few sub-channels been switched off. The appearance of the signal at the input of the $\Delta \Sigma$ modulator is shown in Figure 5.16 below.

The frequency of the clock generator is changed to $\mathrm{f}=131.072 \mathrm{MHz}$ for the MTPR evaluation. The frequency is the multiple of sample frequency of the incoming signal $\mathrm{f}_{\mathrm{S}}=4000 \mathrm{~Hz}$, number of channels/tones $\mathrm{N}_{\mathrm{TONE}}=256$ and the buffer size $\mathrm{N}_{\mathrm{BUF}}=$ 
128. The buffering makes frame-based signal format that is required for the FFT algorithm to compute the signal PSD.

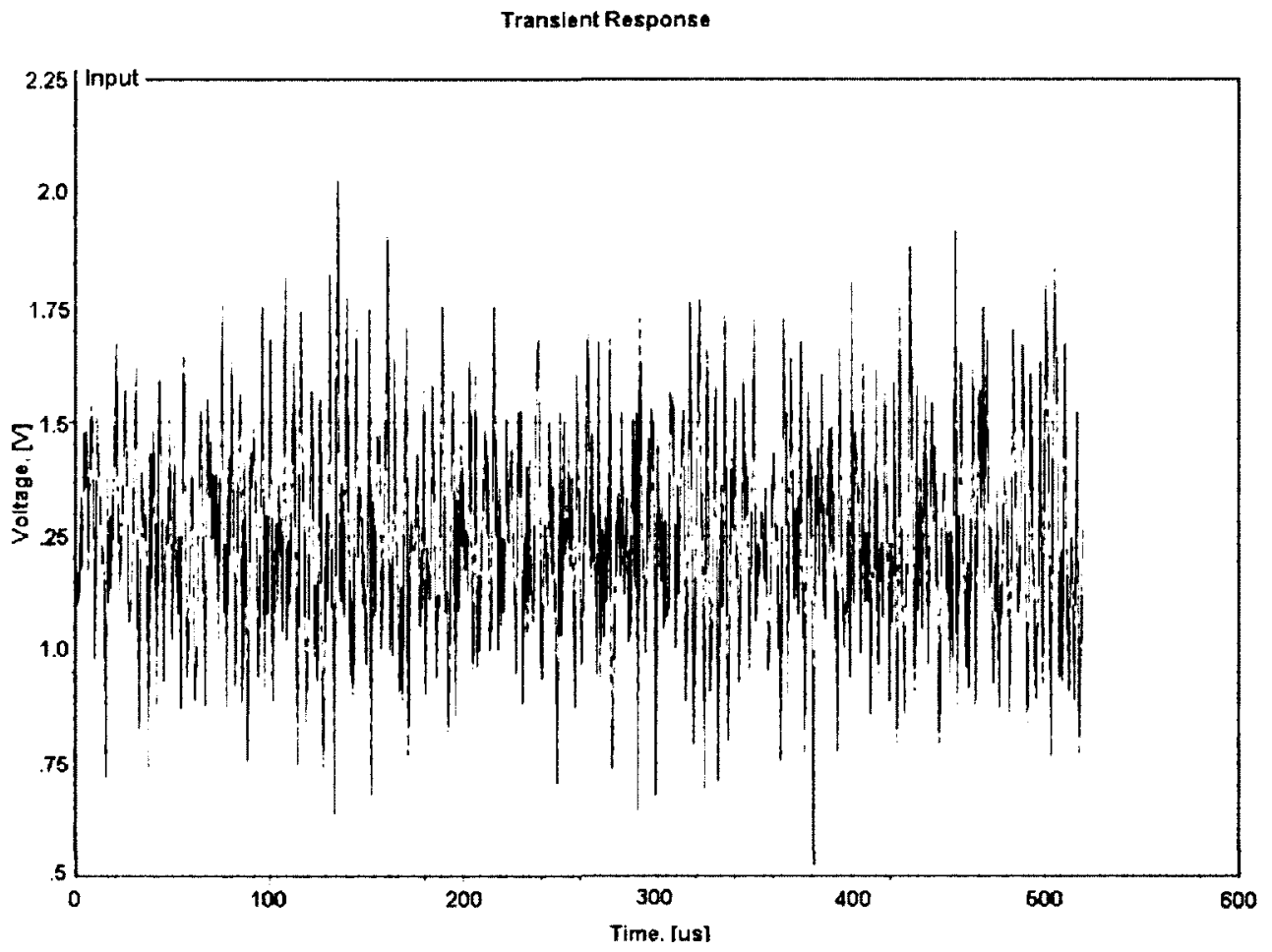

Figure 5.16 DMT Signal Instantiation at the $\Delta \Sigma$ Modulator's Input

The signals at the output of the $\Delta \Sigma$ modulator are saved in an ascii file. The OCEAN script below is used to capture the signal in the file.

ocnPrint(VT(“/Out") ?output"/home/starych/thesis/SigmaDelta/File"

?numberNotation 'none ?precision 14)

Such way of data capture improves the resolution of the data to be processed and so minimizes the signal distortion at the input of the processing jig.

The MTPR value is estimated as the drop of signal power density at the frequencies of missing sub-channels. The bottom level of the power density within the 
drop represents the intrinsic noise of the $\Delta \Sigma$ modulator and inter-modulation products generated when the DMT signal is applied.

The Simulink toolbox for the MTPR evaluation is shown in Figure 5.17 below.

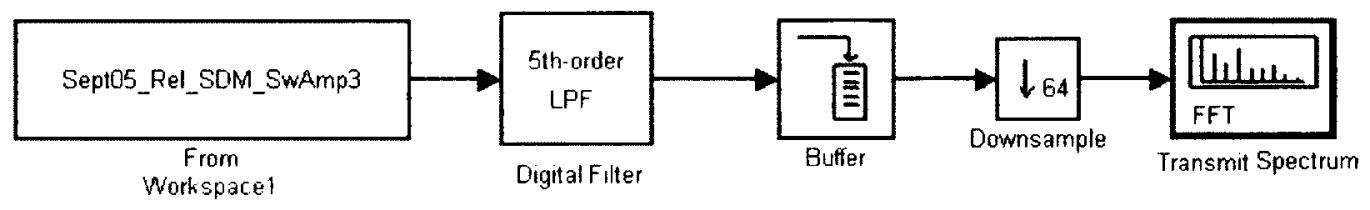

Figure 5.17 ADSL Signal Processor for MTPR Evaluation

The digital low-pass filter of $\mathrm{BW}=1 \mathrm{MHz}$ bandwidth is placed in front of the processor. This filter is not the part of the line driver and is intended to filter out highfrequency noise during the signal processing.

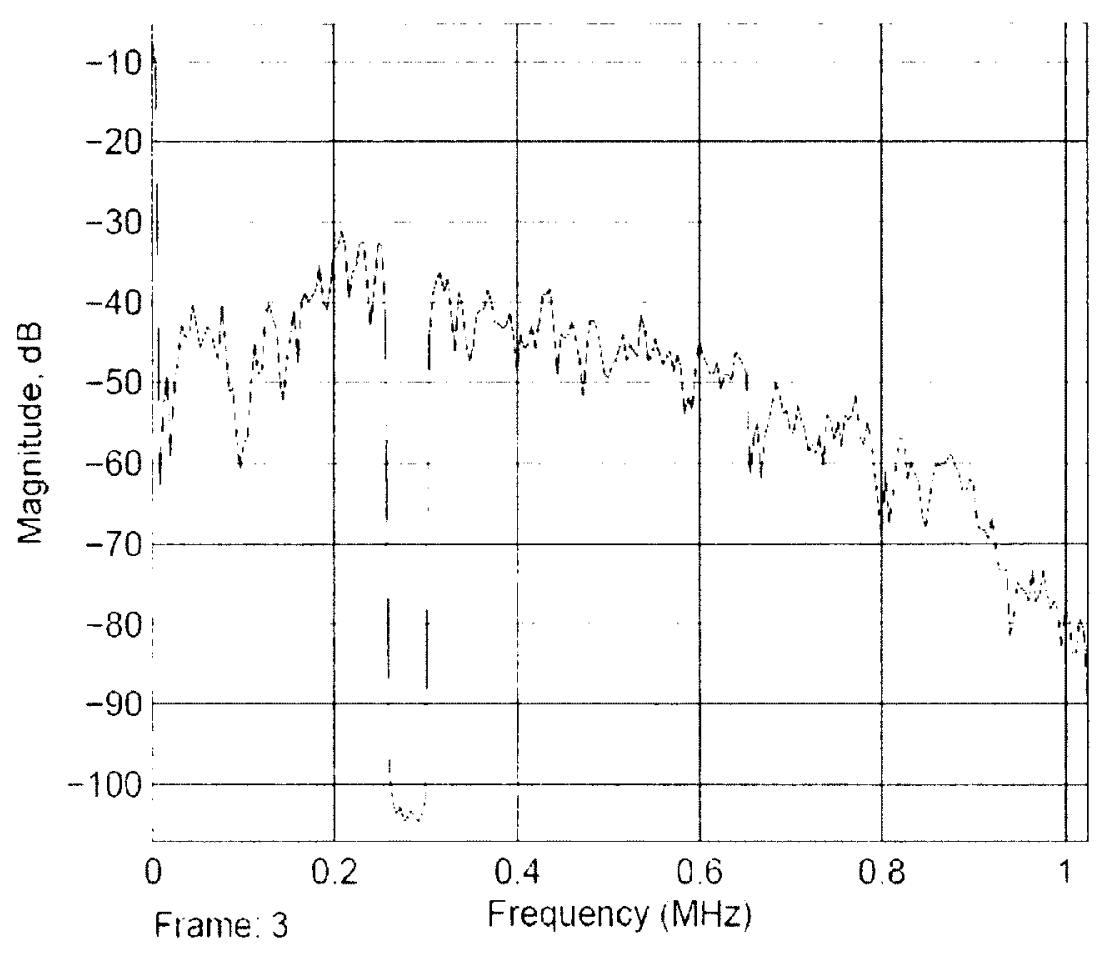

Figure 5.18 MTPR of 2nd-order $\Delta \Sigma$ Modulator 
The result of the $\Delta \Sigma$ modulator circuit simulation is shown in Figure 5.18 above. The MTPR at the output of the modulator designed for this work is about $70 \mathrm{~dB}$. This value is $5 \mathrm{~dB}$ better compared to the corresponding Simulink model of the $\Delta \Sigma$ modulator (see Figure 3.19 in Chapter 3 above).

\subsubsection{Line Driver Characterization}

The line driver is composed of the $\Delta \Sigma$ modulator followed by the power amplifier. The evaluation of SNR, DR and MTPR parameters are done for the whole circuit. Such approach allows estimating the saturation of the line driver's performance due to the switching power amplifier, if necessary, because the performance of the $\Delta \Sigma$ modulator itself was evaluated earlier. The schematic of the line driver is shown in Figure 5.19 below.

The schematic includes the components that belong to the test bench of the line driver. The components shown in Figure 5.19 (the sine-wave generator, the reset-pulse generator and the clock generator) are used to simulate the operation of the line driver for the evaluation of its SNR and DR. The frequency of the clock generator is $f=128$ $\mathrm{MHz}$.

The results of the SNR evaluation for the signal of frequency $f_{\mathrm{IN}}=250 \mathrm{kHz}$ and peak power $\mathrm{P}=100 \mathrm{~mW}$ at the output of the line driver are provided in Figure 5.20 and Figure 5.21 below. 


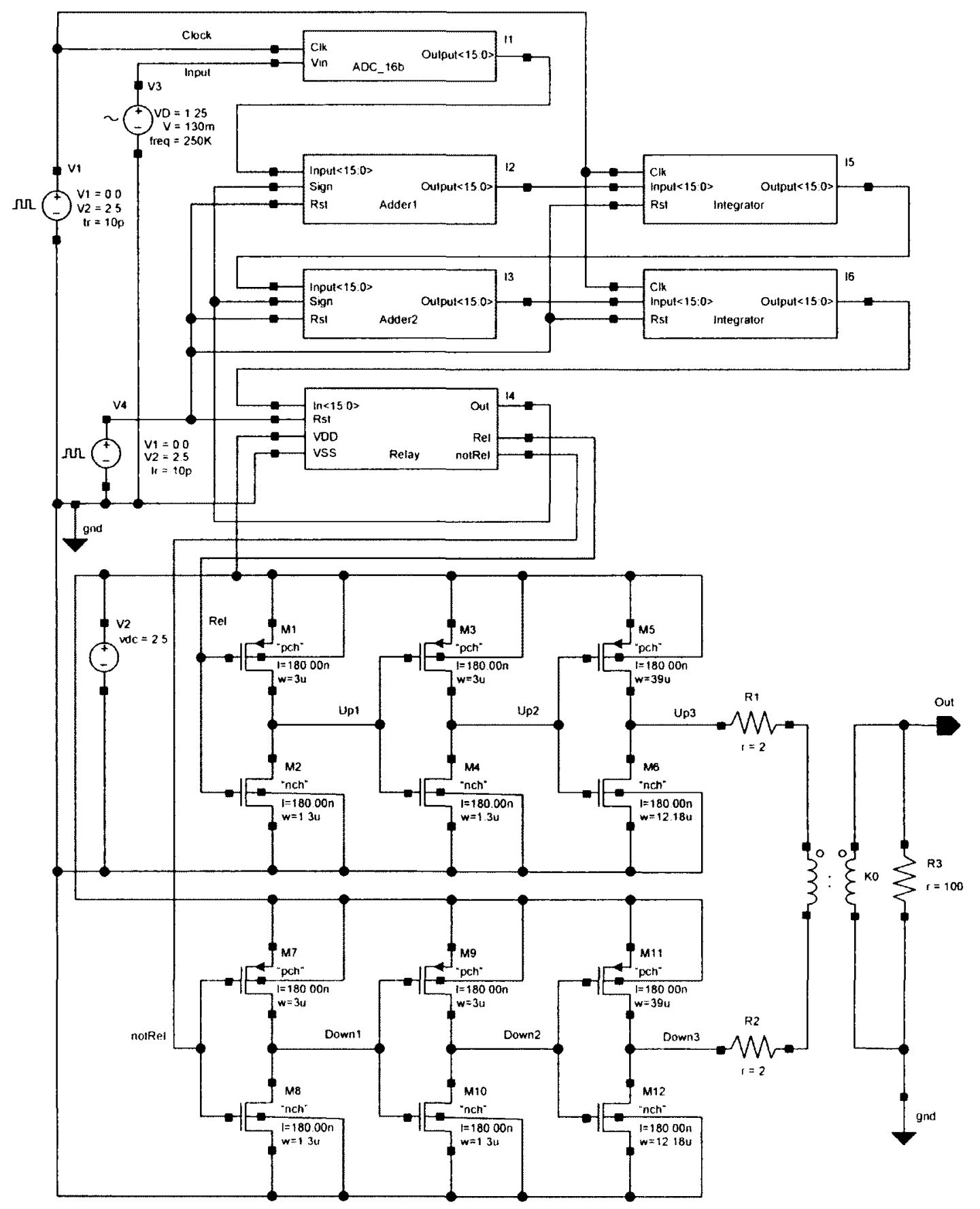

Figure 5.19 ADSL Line Driver Schematic 


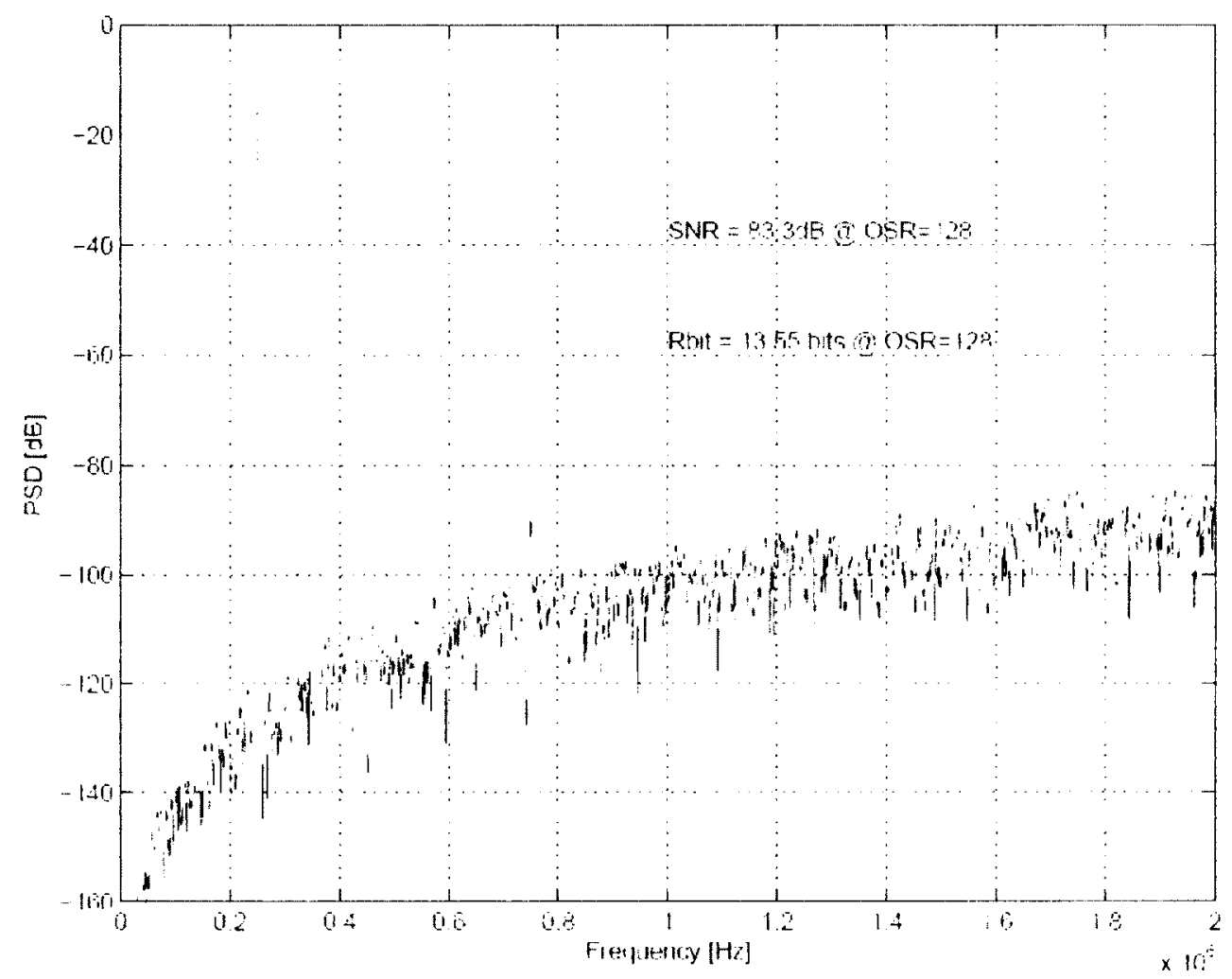

Figure 5.20 Signal Spectrum of the Line Driver with Switching Power Amplifier, $\mathrm{f}_{\mathrm{N}}=250 \mathrm{kHz}, \mathrm{BW}=500 \mathrm{kHz}$

The analysis of the signal's spectrum shows that the level of unwanted harmonics at the output of the line driver is extremely low. The $3^{\text {rd }}$ harmonic at $f=750$ $\mathrm{kHz}$ frequency is observed only. The level of the harmonic is $80 \mathrm{~dB}$ below the PSD level at the signal's main frequency. It is worth to note that no filters are used in the line driver at all.

The Spurious Free Dynamic Range (SFDR) of the signal at the output of the line driver can be estimated as $80 \mathrm{~dB}$ in $1 \mathrm{MHz}$ bandwidth and about $70 \mathrm{~dB}$ in $2 \mathrm{MHz}$ bandwidth. 


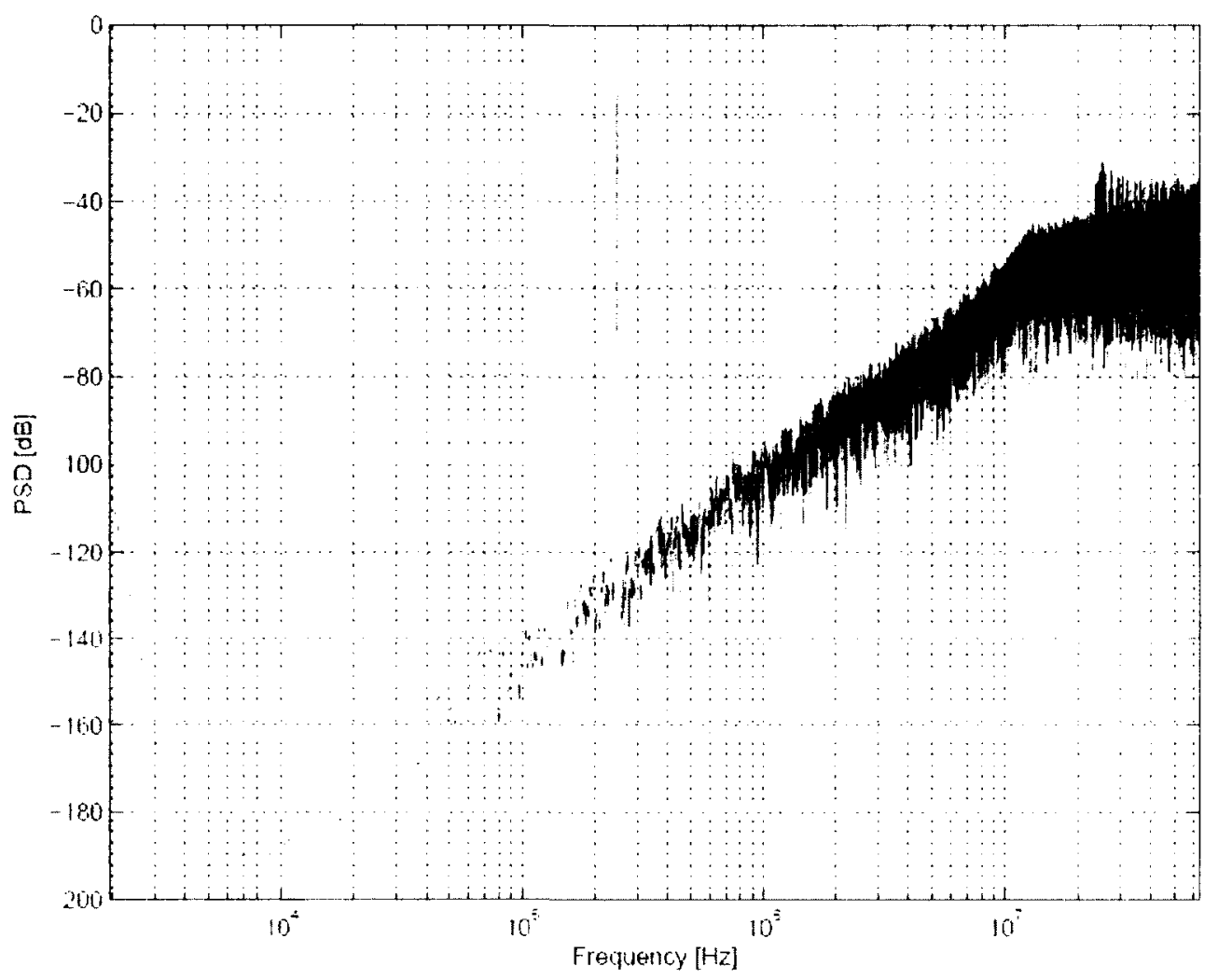

Figure 5.21 Signal Spectrum of the Line Driver with Switching Power Amplifier, $f_{\text {IN }}=250 \mathrm{kHz}, \mathrm{BW}=500 \mathrm{kHz}$

The simulation results in Figure 5.20 and Figure 5.21 are for the switching power amplifier with $\mathrm{R}_{\text {MATCH }}=\mathrm{Rl}=\mathrm{R} 2=2 \mathrm{Ohm}$ resistor. As one can see, the SNR value is $2.5 \mathrm{~dB}$ smaller at the output of the switching power amplifier compared to the signal SNR at the input of the amplifier (see Figure 5.14 above).

The SNR value for the circuit with $\mathrm{R}_{\text {MATCH }}=0 \mathrm{Ohm}$ is the same. The main difference between the circuits with different $R_{\text {MATCH }}$ values is the current density in the switching transistors of the amplifier, $\mathrm{J}=0.67 \mathrm{~mA} / \mu \mathrm{m}$ and $\mathrm{J}=0.60 \mathrm{~mA} / \mu \mathrm{m}$ accordingly as per Table 5 above. This confirms that the current density is not critically high, and 
the unwanted processes like hot-carrier generation that are often associated with high current density, don't contribute into the circuit noise.

The dynamic range of the line driver in Figure 5.19 above is additionally evaluated for the range of signals at the driver's input to make sure that the SNR value depicted in Figure 5.20 is the maximum and provides the correct DR estimation of the line driver. The results are shown in Figure 5.22 below. The circuit demonstrates the dynamic range $\mathrm{DR}=83 \mathrm{~dB}$, which is about $10 \mathrm{~dB}$ less than $\mathrm{SNDR}=94 \mathrm{~dB}$ calculated in Section 3.3 for the $2^{\text {nd }}$-order $\Delta \Sigma$ modulator.

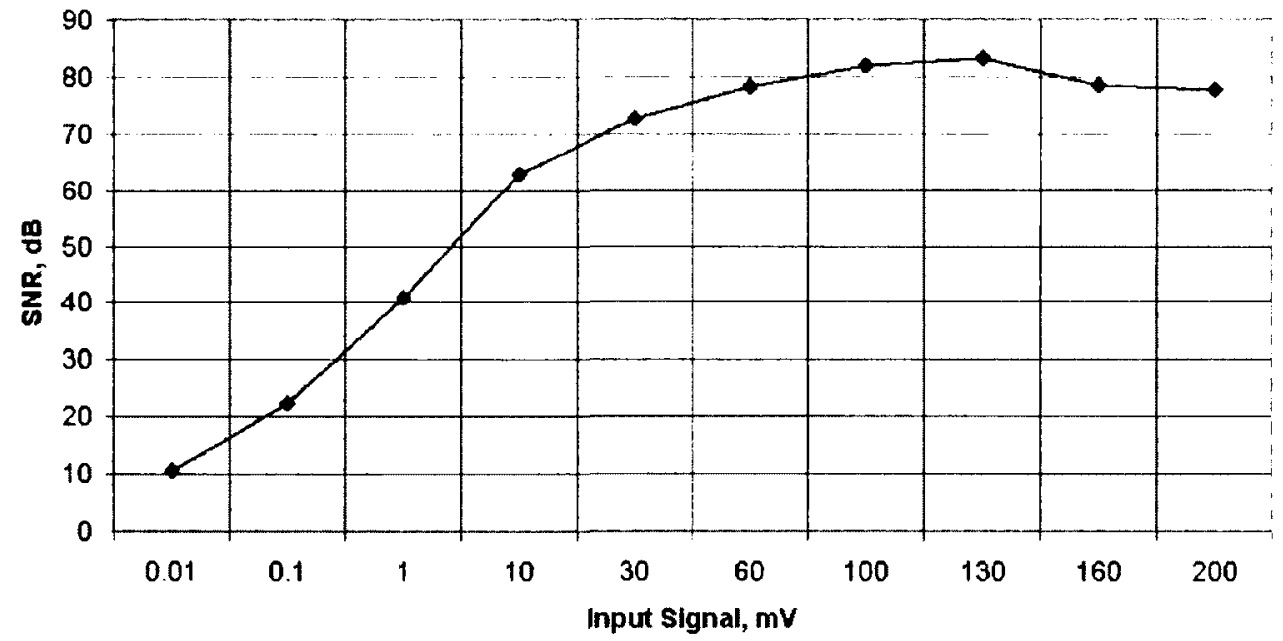

Figure 5.22 Dynamic Range of the Line Driver, $f_{\mathrm{IN}}=250 \mathrm{kHz}, \mathrm{BW}=500 \mathrm{kHz}$

The SNR value in $\mathrm{dB}$ is directly related to the number of bits of the digital signal that is applied to modulate each carrier in the DMT signal:

$$
\mathrm{R}=\frac{\mathrm{SNR}-1.76}{6.02}, \text { bit }
$$

Accordingly, the dynamic range can be plotted as shown in Figure 5.23 below. 


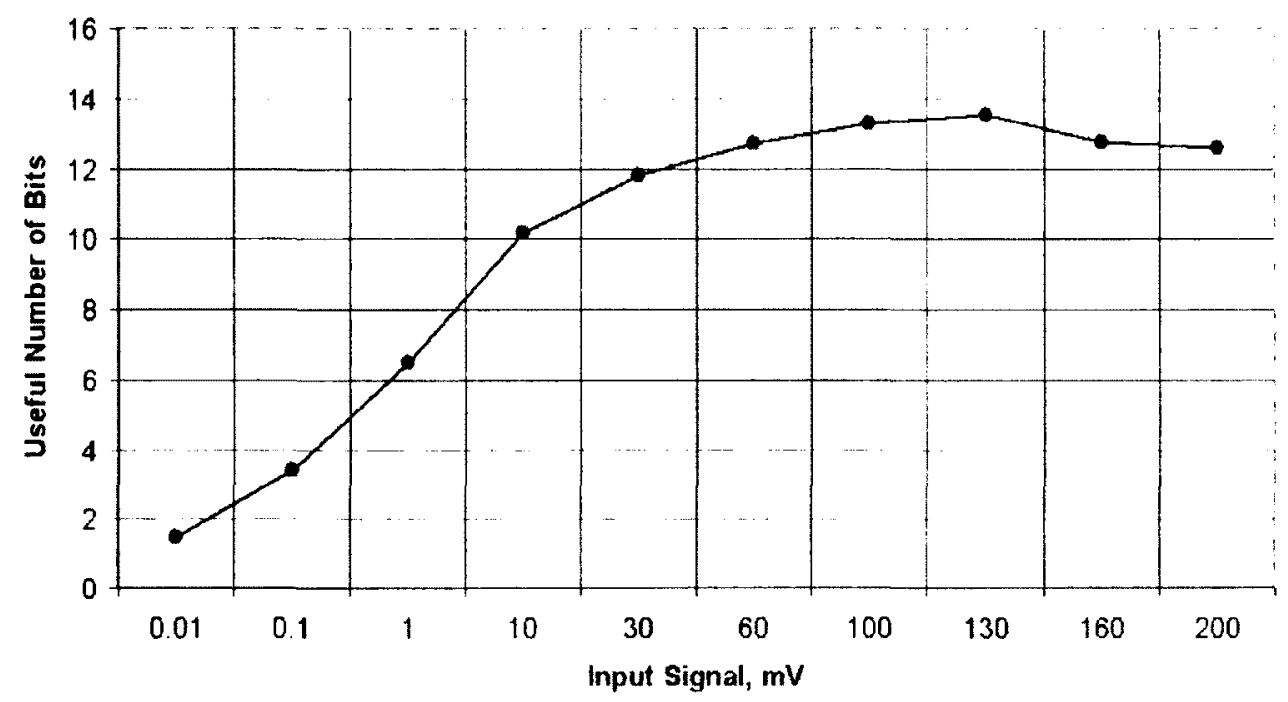

Figure 5.23 Dynamic Range of the Line Driver, $f_{\mathrm{IN}}=250 \mathrm{kHz}, \mathrm{BW}=500 \mathrm{kHz}$

The response of the Line Driver for the input signals in $30-1000 \mathrm{kHz}$ range is studied, and the results of the simulations are provided in Figure 5.24 below.

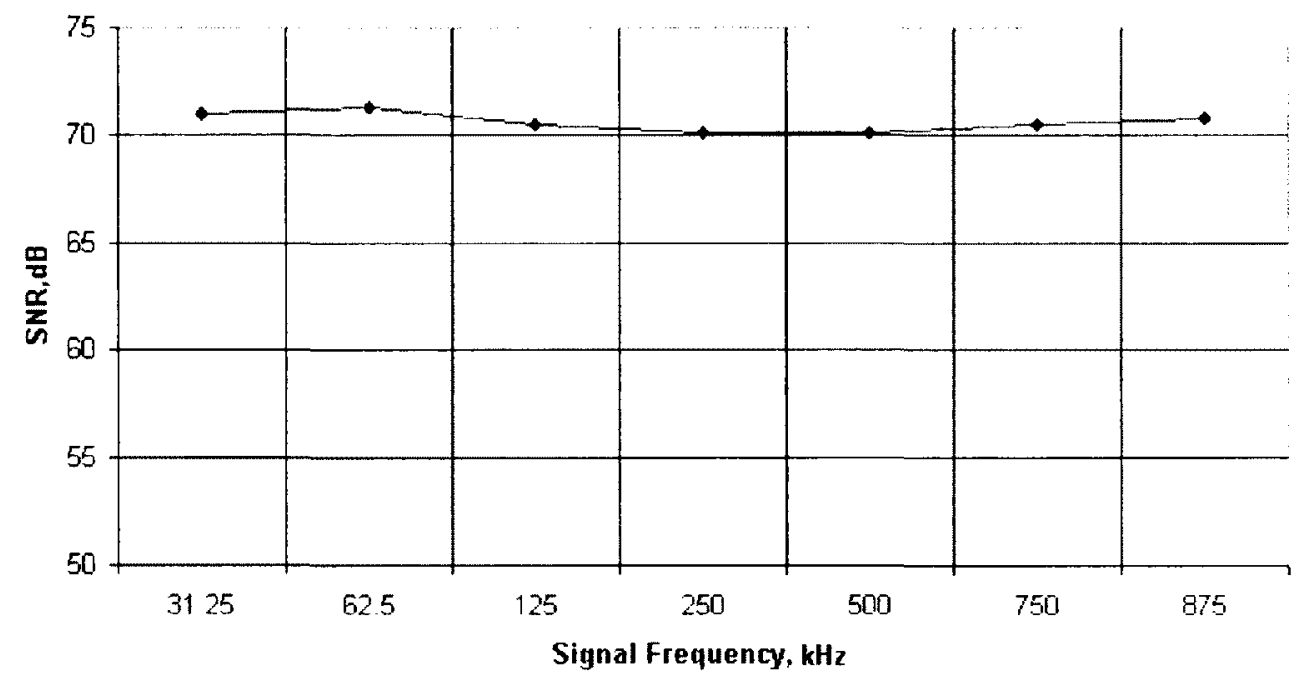

Figure 5.24 Frequency Range of the Line Driver, BW = $1 \mathrm{MHz}$

No performance degradation of the line driver is found within entire ADSL frequency band. The dynamic range in Figure 5.24 is calculated for $1 \mathrm{MHz}$ bandwidth. That is why the SNR values are lower than shown in the relevant figures above. 
The MTPR characterization of the line driver is done with the same test bench as used for the dynamic range study, but with the sine-wave generator replaced for the vpwlf component described in Section 5.3.2.

The frequency of the clock generator is changed to $\mathrm{f}=131.072 \mathrm{MHz}$ for the MTPR evaluation. The frequency is the multiple of sample frequency of the incoming signal $f_{S}=4000 \mathrm{~Hz}$, number of channels/tones $N_{T O N E}=256$ and the buffer size $N_{B U 1}=$ 128. The buffering makes frame-based signal format that is required for the FFT algorithm to compute the signal PSD.

The signal at the output of the line driver is captured using the Ocean script and processed with the Simulink toolbox as explained in Section 5.3.2.

As reported in Section 5.3.2 the MTPR is $70 \mathrm{~dB}$ at the output of the $\Delta \mathrm{E}$ modulator of $2^{\text {nd }}$ order (Figure 5.18 above). The same value MTPR $=70 \mathrm{~dB}$ for $\mathrm{BW}=$ $1 \mathrm{MHz}$ is found for the signal at the output of the switching power amplifier (Figure 5.25 below).

The Line Drivers with $\mathrm{R}_{\mathrm{MATCH}}=0$ Ohms and $\mathrm{R}_{\mathrm{MATCH}}=2$ Ohms are simulated for the MTPR evaluations. Both drivers exhibit the same performance. The switching amplifier itself doesn't degrade the MTPR. This result is somewhat expected as the amplifier is not supposed to change the shape of the signal, and so it doesn't disturb the spectrum of the signal. 


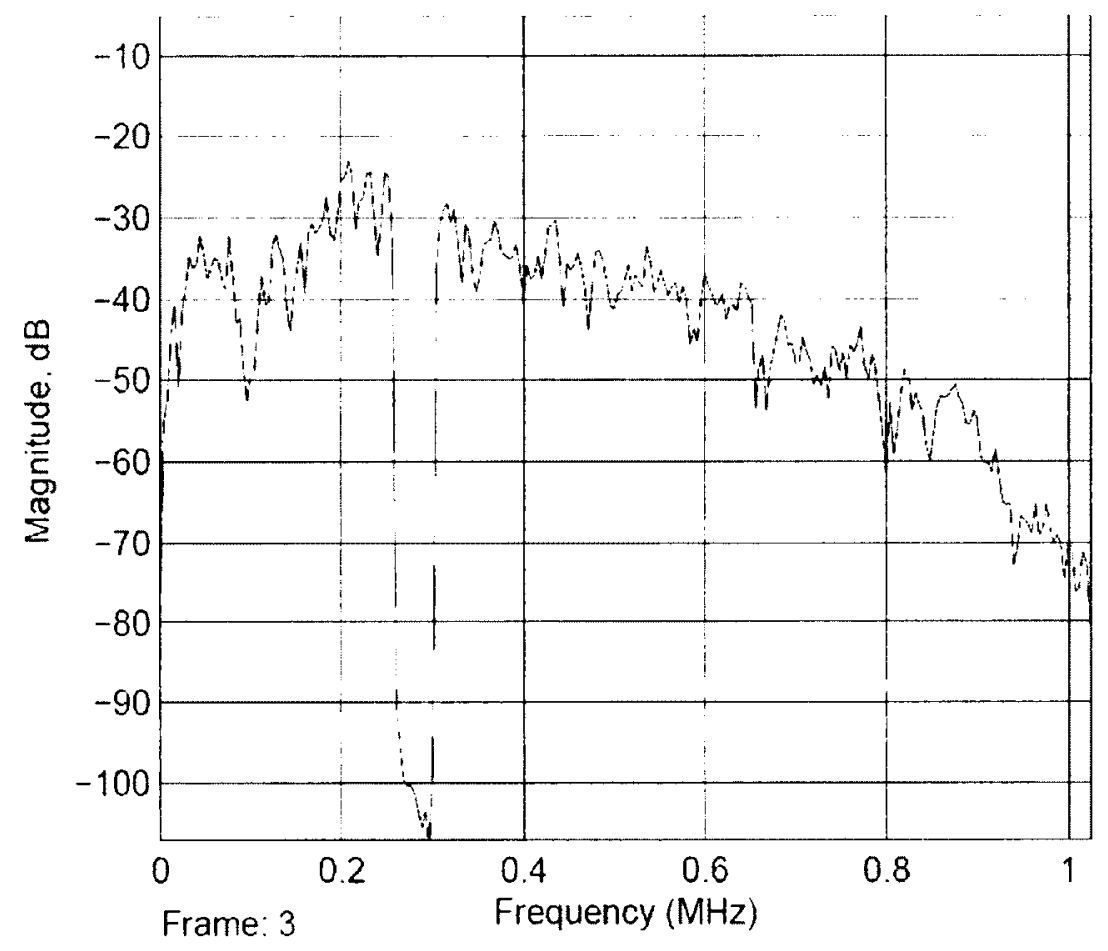

Figure 5.25 MTPR of the ADSL Line Driver 


\section{Conclusions and Future Work}

\subsection{Summary}

The system solution for ADSL line driver is studied. The proposed line driver is composed of the $\Delta \Sigma$ modulator followed by the switching power amplifier. The modulator is implemented as the digital circuit that transforms multi-bit code generated by IFFT DSP into single-bit digital signal. The latter one is amplified by the switching power amplifier and routed into ADSL communication link.

Simulations showed that $2^{\text {nd }}-$ order low-pass $\Delta \Sigma$ modulator is capable to produce the signal suitable for ADSL applications. Simulink model of the modulator exhibits the $\mathrm{DR}=85 \mathrm{~dB}$ for $\mathrm{f}_{\mathrm{IN}}=250 \mathrm{kHz}$ signal in $\mathrm{BW}=500 \mathrm{kHz}$ bandwidth. The clock frequency is approximately $130 \mathrm{MHz}$. The frequency is feasible for $0.18 \mu \mathrm{m}$ CMOS technology that is preliminarily selected for the silicon implementation of the line driver.

The Simulink model of $2^{\text {nd }}$-order $\Delta \Sigma$ modulator provides suitable performance when DMT signal is applied at its input. The MTPR value is approximately $70 \mathrm{~dB}$ that is $5 \mathrm{~dB}$ higher than the maximum MTPR expected when 256-QAM DMT signal is employed.

The modules of $\Delta \Sigma$ modulator are designed in Verilog HDL and compiled. The corresponding components are simulated in Cadence Virtuoso Design Environment together with switching power amplifier. The switching amplifier is composed of three CMOS invertors connected in series. The transistors of the last switching stage are made large enough to provide the signal of $\mathrm{P}_{\mathrm{L}}=100 \mathrm{~mW}$ power in $\mathrm{R}_{\mathrm{L}}=100 \mathrm{Ohm}$ load. 
The matching circuitry is included in the switching amplifier to improve the efficiency of the transmission chain. The power efficiency of the switching power amplifier is approximately $60 \%$ as per simulation results.

The simulations show that the line driver can provide the $\mathrm{DR}=83 \mathrm{~dB}$ in $\mathrm{BW}=$ $500 \mathrm{kHz}$ bandwidth for the signals in $30-1000 \mathrm{kHz}$ frequency range. The MTPR value is $70 \mathrm{~dB}$ in $\mathrm{BW}=1 \mathrm{MHz}$ bandwidth.

Spurious Free Dynamic Range SFDR $=80 \mathrm{~dB}$ in $\mathrm{BW}=1 \mathrm{MHz}$ and SFDR $=70$ $\mathrm{db}$ in $2 \mathrm{MHz}$ bandwidth. The only spurious signal observed in $\mathrm{BW}=2 \mathrm{MHz}$ is the $3^{\mathrm{rd}}$ harmonic of the main signal. The level of the harmonic is $80 \mathrm{~dB}$ less than the level of the signal main harmonic.

The proposed solution for the ADSL line driver seems very promising. Being completely digital, it allows using standard CMOS technology for the circuit fabrication. The $\Delta \Sigma$ modulator doesn't include any analog modules that are usually associated with complicated calibration techniques and circuit trimming.

\subsection{Results Comparison}

The $\Delta \Sigma$ modulator as a data formatter and switching power amplifier are not usually studied together as the solution for the ADSL line driver. This is one of the reasons why the number of relevant technical publications is very low. That is why the publications that are referred below are sorted out for two groups. The first group includes the technical papers that study the $\Delta \Sigma$ modulators, which are close to the one presented in this work from view point of architecture or application. The second group includes the publications that discuss high-efficient line drivers/transmitters that can be 
used for ADSL communication links regardless of their circuitry solutions and the data formatters preceding the amplification stages.

Most $\Delta \Sigma$ modulators, which are discussed in professional technical publications, are designed as analog circuits. The authors are forced to use high-order architectures of the modulators to get the required specifications (dynamic range and $\mathrm{SNR}$ ). As the $\triangle \mathrm{\Sigma}$ modulators of the order of three and higher are conditionally stable and require special means to prevent oscillations, so-called Multi-stage Noise Shaping (MASH) topologies are often used. MASH $\Delta \Sigma$ modulators are the modulators of $1^{\text {st }}$ and $2^{\text {nd }}$ - order connected in series. 2-1-1 topology is very popular solution as it provides necessary performance while being unconditionally stable and relatively simple in its implementations. Almost all $\Delta \Sigma$ modulators listed in Table 6 below are designed as MASH devices.

The modulators in the table are analog circuits. The integrators are designed based switched-capacitor technique. Although the modulators are built as CMOS devices, extra technology extensions are usually used to implement the circuit elements, for example, Metal-Insulator-Metal fabrication process to build the capacitors. Trimming is usually required to fabricate the circuit components with necessary accuracy.

The integrator designed for this work is pure digital circuit that can be fabricated with generic CMOS process without any technology extensions that might be required for the implementation of analog components. The circuit is not locked to some particular CMOS technology. The only limitation is the signal delay. It is determined above that $0.18 \mu \mathrm{m}$ CMOS seems to be suitable for the $\Delta \Sigma$ modulator studied in this 
work. More advanced technology, for example, $90 \mathrm{~nm}$ CMOS can be used as well as it provides less signal delays. Required performance is gotten with low-order modulator topology due to high sampling frequency and, accordingly, high OSR. The circuit performance is tailored for ADSL application only. That is why the circuit study is done for the signal frequency range up to $1 \mathrm{MHz}$ only.

Table $6 \Delta \Sigma$ Modulators

\begin{tabular}{|c|c|c|c|c|c|c|}
\hline Reference & Topology & $\begin{array}{c}\text { Power } \\
\text { Supply, } \\
\text { [V] }\end{array}$ & OSR & $\begin{array}{c}\text { Sample } \\
\text { Frequency, } \\
|\mathrm{MHz}|\end{array}$ & $\begin{array}{l}\text { DR, } \\
{[\mathrm{dB}]}\end{array}$ & Note \\
\hline$|15|$ & $2-1-1$ & 3.3 & 24 & 52.8 & 87 & $\begin{array}{c}0.5 \mu \mathrm{m} \text { CMOS } \\
\text { analog SC } \\
\text { integrators }\end{array}$ \\
\hline$|16|$ & $2-1-1$ & 2.5 & 32 & 70.4 & 85 & $\begin{array}{l}0.25 \mu \mathrm{m} \mathrm{CMOS} \\
\mathrm{MTPR}=81 \mathrm{~dB} \\
\mathrm{BW}=2.2 \mathrm{MHz}\end{array}$ \\
\hline$[17]$ & $2-1-1$ & 1.8 & 24 & 50 & 70 & $\begin{array}{c}0.18 \mu \mathrm{m} \text { CMOS } \\
\text { analog SC } \\
\text { integrators }\end{array}$ \\
\hline$|18|$ & 4 & 1.8 & 10 & 200 & 82 & $\begin{array}{c}0.18 \mu \mathrm{m} \text { CMOS, } \\
\text { analog SC } \\
\text { integrators, } \\
\mathrm{BW}=10 \mathrm{MHz}\end{array}$ \\
\hline This work & 2 & 2.5 & 128 & 128 & 83 & $\begin{array}{c}0.18 \mu \mathrm{m} \text { CMOS, } \\
\text { digital circuit }\end{array}$ \\
\hline
\end{tabular}

The $\Delta \Sigma$ modulator designed for this work demonstrates satisfactory performance while being robust and having simple topology. This becomes possible due to high speed of digital circuits that is one of the advantages of modern CMOS technologies. 
It should be noted that the top performance of the $\Delta \Sigma$ modulator is not the main goal of the design. The proposed modulator is expected to meet the ADSL specifications with some margin in order to be used as the data formatter for the following power amplifier. The power efficiency of the amplifier, which is used as the output stage of the ADSL line driver, is considered as more important figure of merit of the device designed for this work.

The switching power amplifier, which is very natural solution for the CMOS technologies, is proved to be one of the most power-efficient circuits for DSL links. Almost all the references provided in Table 7 below, study the implementations of the switching amplifier, which output stage is made as standard PMOS-NMOS stacked transistor pair. With regards to the switching power amplifiers, this circuit is often referred as half H-bridge topology.

Although the circuitry made of NMOS-PMOS transistor pole seems simple, the designers propose different implementations of it to address the technology and application related restrictions.

SOPA concept proposed in [3] is one of the first attempts to resolve the problem of low power-efficiency of Class AB power amplifiers for high CF DSL signals. It is remarkable that the authors decided to look for the solution within CMOS framework. The SOPA topology proposed as the data formatter allows using switching power amplifier at the output of the circuit. The resultant circuit outperforms the analog power amplifiers and meets ADSL and VDSL requirements. 
The circuit studied in $[29]$ is also SOPA data formatter with high-voltage output stage, where standard low-power CMOS components are used. This solution allows reducing the high current in the output stage.

Table 7 Switching Power Amplifiers

\begin{tabular}{|c|c|c|c|c|c|c|}
\hline Reference & [3] & [29] & {$[30]$} & $|35|$ & {$[36]$} & $\begin{array}{l}\text { This } \\
\text { work }\end{array}$ \\
\hline Architecture & $\begin{array}{c}\text { Half } \\
\text { H-bridge }\end{array}$ & $\begin{array}{c}2 \times 5 \\
\text { stacked } \\
\text { transistors }\end{array}$ & $\begin{array}{c}\text { Half } \\
\text { H-bridge, } \\
\text { differential }\end{array}$ & $\begin{array}{c}\text { Half } \\
\text { H-bridge, } \\
\text { differential }\end{array}$ & $\begin{array}{c}\text { Half } \\
\text { H-bridge, } \\
\text { differential }\end{array}$ & $\begin{array}{c}\text { Full } \\
\text { H-bridge }\end{array}$ \\
\hline $\begin{array}{c}\text { Power } \\
\text { Supply, V }\end{array}$ & 3.3 & 5.5 & 3.3 & 100 & 3 & 2.5 \\
\hline $\begin{array}{c}\text { Efficiency, } \\
\%\end{array}$ & 47 & 42 & 22.8 & 13.1 & 77 & 60 \\
\hline MTPR, dB & 56 & 58 & 31 & 40 & NA & 70 \\
\hline Application & $\begin{array}{l}\text { ADSL } \\
\text { Line } \\
\text { Driver }\end{array}$ & $\begin{array}{c}\text { ADSL2+ } \\
\text { Line } \\
\text { Driver }\end{array}$ & $\begin{array}{l}\text { ADSL } \\
\text { VDSL } \\
\text { Line } \\
\text { Driver }\end{array}$ & $\begin{array}{l}\text { ADSL } \\
\text { Line } \\
\text { Driver }\end{array}$ & $\begin{array}{c}\text { Audio } \\
\text { Class D } \\
\text { PA }\end{array}$ & $\begin{array}{c}\text { ADSL } \\
\text { Line } \\
\text { Driver }\end{array}$ \\
\hline
\end{tabular}

The line driver discussed in [30] employs the output stage built of NMOSNMOS totem pole in order to minimize the gate capacitance and delay. This allows the designer to get wider operational bandwidth of the device; however, the power efficiency of the line driver got lower.

The problem of high current in high-power circuits is resolved directly in [35]. The authors propose switching line driver designed for high-voltage extension of 
standard $0.7 \mu \mathrm{m}$ CMOS technology. The power efficiency of the circuit is increased compared to Class $\mathrm{AB}$ power amplifiers, but it is still lower than reported in [3].

The $\Delta \Sigma$ modulator is proposed as the data formatter of the line driver for audio signal in [36]. The output stage of the line driver is designed as the switching power amplifier. The $4^{\text {th }}$-order $\Delta \Sigma$ modulator uses SC analog integrators with high switching frequency.

The devices referred in Table 7 above are designed for different technical specifications, and so it is not quite fair to compare their performance relative to each other. However, the references give the overall impression about the progress in the design of power-effective DSL line drivers.

\subsection{Thesis Contribution}

The main goal of this work is to build high efficient ADSL line driver driven by digital data encoder. As the result, the circuit composed of $\Delta \Sigma$ modulator followed by switching power amplifier is proposed.

Such system solution has been discussed in technical community. However, it is not considered as the real opportunity for xDSL applications. That is why probably no publications, which would discuss this solution in professional manner, are found in technical literature.

It should be noted that the $\Delta \Sigma$ modulator is very popular circuit. It is usually used in Analog-to-Digital Converters. The $\Delta \Sigma$ modulator as part of ADC module is often built as an analog device, mostly based on switched-capacitor integrators. No pure 
digital implementation of the $\Delta \Sigma$ modulator or HDL code of the $\Delta \Sigma$ modulator of $2^{\text {nd }}$ order and higher are found in the technical literature at all.

The contributions of the thesis work can be defined as follows.

- The system solution for the ADSL digital line driver, which is composed of the $\Delta \Sigma$ modulator and switching amplifier, is justified.

- The architecture of the $\Delta \Sigma$ modulator is defined by thorough simulations of Simulink model of the modulator.

- The Verilog code of the $\Delta \Sigma$ modulator is written, compiled and integrated in Cadence Virtuoso Design Environment..

- The application of switching power amplifier for ADSL signal is justified.

- The test bed for ADSL signal is designed.

- High DR and MTPR values have been demonstrated.

- The power efficiency of the switching line driver is estimated. It is found that the efficiency is close to the theoretical level of $50 \%$.

- The proposed line driver demonstrates very low level of unwanted harmonics in the output signal spectrum. The result is gotten without using any filters at the output of the driver.

It is expected that the proposed solution is applicable not only to DMT-based ADSL links, but also can be used in any other Ultra-Wide Band (UWB) communication systems that employ OFDM technology. 


\subsection{Recommendations and Future Work}

The current work should be considered as comprehensive, but still paper study of the ADSL line drivers. Proposed system solution is very promising; however, its feasibility can be finally judged only based on the test results of the real device. The implementation of the proposed line driver on the silicon is the very first thing to do.

It is probably useful to make the $\Delta \Sigma$ modulator design, as the first approach, in an FPGA prototype. The FPGA is quite powerful and flexible platform that allows the designer to catch the weak sides of the design at its early stage. When the design of the $\Delta \Sigma$ modulator is proved, the circuit can be fabricated as an ASIC, if necessary.

VLSI circuits when fabricated have very limited possibilities with regards to the changes of the circuits that might be found necessary during the circuit troubleshooting and evaluation. That is why the thorough verification and post-layout simulation of the synthesized circuit should be completed.

Post-layout simulation is very important for the design of the $\Delta \Sigma$ modulator and, in particular, for the switching power amplifier. The large size of the amplifier's switching transistors is associated with large parasitic capacitances that spread over the circuitry. They can significantly affect the performance of the amplifier. However, the successful designs of high-power switching amplifiers are reported in many publications. This proves that the problem of the parasitic capacitances can be resolved.

The other challenge associated with the design of the switching power amplifier is high current density. It could reduce the reliability of the circuit if the conductors are not made wide enough. Also too high currents can generate extra noise in the circuitry. 
This factor is essential for the receivers, and so it is better to keep it in mind as well when the line driver is being designed.

$2^{\text {nd }}$-order $\Delta \Sigma$ modulator has been studied in this work. It provides suitable performance the ADSL communication links. The other topologies of the modulator can be studied in order to determine if they provide better performance. The $\Delta \Sigma$ modulator architecture suitable for VDSL standard can be defined as well. 


\section{References}

[1] Jan Sevenhans et al., "Driving the DSL Highway: high speed, high density, low power, low cost", Proceedings of the $28^{\text {th }}$ European Solid-State Conference, ESSCIRC, pp.555-562, 2002.

[2] S.-S. Le, "Integration and System Design Trends of ADSL Analog Front Ends and Hybrid Line Interfaces", Proceedings of the IEEE Custom Integrated Circuits Conference, pp.37-44, 2002.

[3] T. Piessens, M.Steyaert, "Highly Efficient xDSL Line Drivers in 0.35-um CMOS Using a Self-Oscillating Power Amplifier", IEEE Journal of SolidState Circuits, Vol. 38, No. 1, pp.22-29, 2003.

14] S. Saponara, L. Serafini, L. Fanucci, "Low-power FFT/IFFT VLSI Macro Cell for Scalable VDSL Modem", Proceedings of 3rd IEEE International Workshop on SoC for Real-Time Applications, 2003.

[5] M. Nava, C. Del-Toso, "A Short Overview of the VDSL System Requirements”, IEEE Communication Magazine, Vol.40, pp.82-90, 2002.

16] O.W. Ibraheem, N.N. Khamiss, "Design and Simulation of Assymmetric Digital Subscriber Line (ADSL) Modem", 3rd International Conference on Information and Communication Technologies: from Theory to Applications, pp. 1-6, 2008. 
[7] Asymmetric Digital Subscriber Line (ADSL) Transceivers, ITU-T Recommendation G.992.1, 1999.

[8] Splitterless Asymmetric Digital Subscriber Line (ADSL) Transceivers, ITUT Recommendation G.992.2, 1999.

[9] M. Debbah, "Short Introduction in OFDM", Alcatel-Lucent, White paper.

[10] J.Candy, "The Structure of Quantization Noise from Delta-Sigma Modulators", IEEE Transactions on Communications, Vol. 29, No.9, 1989.

$111]$ N. Maghari, S. Kwon, G. C. Temes, U. Moon, "Sturdy MASH $\Delta-\Sigma$ Modulator", Electronics Letters, October 2006, Vol. 42, No.22.

[12] S. Brigati, F. Francescony, P.Malcovati, F.Maloberti, "Modeling Bandpass Sigma-Delta Modulators in Simulink" Proceedings of International Workshop on ADC Modeling and Testing, IW ADC 2000, Vienna, Austria, September 2000 .

[13] C.-I. Lao, H.-I. Ieong, K.-F. Au, K.-H. Mok, S.-P. U, R. Martins, "A 10.7 MHz Bandpass Sigma-Delta Modulator Using Double Delay Single-opamp SC Resonator with Double-Sampling", Proceedings of International Symposium on Circuits and Systems ISCAS'03, IEEE, Vol. 1, pp.10611064, 2003.

[14] J. M. de la Rosa, B. Perez-Verdu, F. Medeiro, A. Rodriguez-Vazquez "A 2.5 MHz 55dB Switched-Current Bandpass Delta-Sigma Modulator for AM 
Signal Conversion", Proceedings of European Solid-State Circuits

Conference, pp.156-159, 1997.

[15] Y. Geerts, A. Marques, M. Steyaert, W. Sansen, “A 3.3-V, 15-bit, DeltaSigma ADC with Signal Bandwidth of 1.1 MHz for ADSL Applications", IEEE Journal of Solid-State Circuits, Vol.34, No.7, July 1999.

[16] R. del Rio, J. M. de la Rosa, B. Perez-Verdu, M. Delgado-Restituto, R. Dominguez-Castro, F. Medeiro, A. Rodriguez-Vazquez "Highly Linear 2.5V CMOS Delta-Sigma Modulator for ADSL+", IEEE Transactions on Circuits and Systems I: Regular Papers, Vol. 51, pp.47-62, 2004.

[17] M. Safi-Harb, G. W. Roberts "Low-Power Delta-Sigma Modulator for ADSL Applications in Low-Voltage CMOS Technology", IEEE Transactions on Circuits and Systems I: Regular Papers, Vol. 52, pp.2075$2089,2005$.

[18] P. Balmelli, Q. Huang "A 25-MS/s 14-b 200-mW $\Sigma \Delta$ Modulator in 0.18$\mu \mathrm{m}$ CMOS”. IEEE Journal of Solid-State Circuits, Vol. 39, No. 12, 2004.

[19] J. B. Hughes, I. C. Macbeth, D. M. Pattullo "Second Generation SwitchedCurrent Signal Processing", IEEE International Symposium on Circuits and Systems, Vol. 4, pp.2805-2808, 1990.

|20| J. B. Hughes, K. W. Moulding "Switched-Current Signal Processing for Video Frequencies and Beyond", IEEE Journal of Solid-State Circuits, Vol.28, No.3, March 1993. 
[21] N. Tan, S. Eriksson "A Low-Voltage Switched-Current Delta-Sigma Modulator”, IEEE Journal of Solid-State Circuits, Vol. 30, No. 5, 1995.

[22] N. Khitouni, S. Boujelben, M. Masmoudi "Switched Current Integrator for Second Order Sigma Delta Modulator", The $16^{\text {th }}$ International Conference on Microelectronics, ICM 2004 Proceedings, pp.595-599, 2004.

[23] S. Masmoudi, M. Fakhfakh, M. Loulou, N. Masmoudi, L. Loumeau "A CMOS $80 \mathrm{MHz}$ Low-pass Switched-Current Fourth Order Sigma Delta Modulator", IEEE International Conference on Sensors, Circuits and Instrumentation Systems, Hammamet, Tunisia, 2007.

[24] N. Moeneclaey, A. Kaiser "Design Techniques for High-Resolution Current-Mode Sigma-Delta Modulators, IEEE Journal of Solid-State Circuits", Vol. 32, No. 7, 1997.

[25] J. Hughes, A. Worapishet, C. Toumazou "Switched-Capacitors versus Switched-Current: a Theoretical Comparison", The 2000 IEEE International Symposium on Circuits and Systems, ISCAS, Proceedings, Vol. 2, pp.409$412,2000$.

[26] Asymmetric Digital Subscriber Line Transceivers 2 (ADSL2), ITU-T Recommendation G.992.3, 2002.

[27] S. Brigati, F. Francesconi, P. Malcovati, D. Tonietto, A. Baschirotto, F. Maloberti, "Modeling Sigma-Delta Modulator Non-Idealities in Simulink", Proceedings of the $19^{\text {th }}$ International Symposium on Circuits and Systems, ISCAS'99, Vol.2, pp.384-387, 1999. 
[28] S. R. Norsworthy, R. Schreier, G. C. Temes, Delta-Sigma Data Converters, Theory, Design and Simulation, New York: IEEE Press, 1997.

[29] B. Serneels, M.Steyaert, W. Dehaene, "A 237mW aDSL+ CO Line Driver in Standard 1.2V 0.13 $\mu \mathrm{m}$ CMOS", Digest of Technical Papers, IEEE International Solid-State Circuits Conference, ISSCC 2007, pp.524-619, 2007.

[30] S. Maugham, R. Henderson, "A Wide Band CMOS Class-D Line Driver for Wireline Communication", $53^{\text {rd }}$ IEEE International Midwest Symposium on Circuits and Systems (MWSCAS), pp.769-772, 2010.

[31] $0.18 \mu \mathrm{m}$ CMOS Process (CS80A), http://www.fujitsu.com/downloads/MICRO/fma/pdf/csm80a.pdf.

[32] Products: ASIC (Gate Arrays), http://www.epson.jp/device/semicon e/product/asic/gatearray.

[33] M. M. Mano, C. R. Kime, "Logic and Computer Design Fundamentals", 4th Edition, Pearson Prentice Hall, 2008.

[34] TSMS 0.18 $\mu \mathrm{m}$ Logic IP6M Salicide 1.8V/3.3V Design Rule, Doc. No. TA-10A5-4001 (T-018-LO-DR-001), Rev.2.2.

[35] V. de Gizelle, J. Doutreloigne, A. van Calster, "A High-Voltage, $75 \mathrm{~mW}$ Switching ADSL Line-Driver", The $17^{\text {th }}$ International Conference on Microelectronics, ICM 2005, p.5, 2005. 
[36] K. Kang, J. Ron, Y. Choi, H. Nam, S. Lee, “Class-D Audio Amplifier Using 1-Bit Forth-Order Delta-Sigma Modulation", IEEE Transactions on Circuits and Systems - II: Express Briefs, Vol. 55, No. 8, 2008. 


\section{Appendix A - Matlab Processing Toolbox}

The original files can be found in

http://www.mathworks.com/matlabcentral/fileexchange/25811-sdtoolbox-2.

The lines of the files modified for the purpose of this work are noted as adjusted for ADSL case.

\section{A.1 SDM Main Processor}

$\%$

$\%$ This file processes the results of simulation of 2nd Order Sigma-Delta

$\%$ Modulator (SDM_Thesis.mdl).

$\%$ 1. Plots the Power Spectral Density of the bit-stream

$\%$ 2. Calculates the SNR

$\%$ 3. Calculates histograms at the integrator outputs

\%

clear

to=clock;

bw $=500000 ; \%$ Bandwidth, adjusted for ADSL case

$R=128 ; \quad \%$ OSR, adjusted for ADSL case

Fs $=2^{*} R^{*} b w ; \%$ Sampling frequency

$\mathrm{Ts}=1 / \mathrm{Fs}$;

$N=65536 ; \quad \%$ Samples number

$\mathrm{L}=\mathrm{N}$;

nper $=128 ; \%$ adjusted for ADSL case

Fin $=n p e{ }^{*} \mathrm{Fs} / \mathrm{N}$; \% Input signal frequency

Ampl=0.159; \%Input signal amplitude, adjusted for ADSL case

Ntransient $=0$

$\%$ Modulator coefficients 
echo on;

b1 $=0.5 ; \quad \%$ adjusted for ADSL case

b2 $=0.5 ; \quad \%$ adjusted for ADSL case

Vref $=0.5 ; \quad \%$ adjusted for ADSL case

Amax $=0.75 ; \%$ Op-amp saturation value [V], adjusted for ADSL case echo off;

finrad=Fin*2* pi; \% Input signal frequency in radians

s0 $=$ sprintf( ${ }^{(* \star}$ Simulation Parameters **');

$\mathrm{s} 1=\operatorname{sprintf(}\left(\mathrm{Fs}(\mathrm{Hz})=\% 1.0 f^{\prime}, \mathrm{Fs}\right)$;

s2=sprintf(' Ts(s)=\%1.6e',Ts);

s3=sprintf(' Fin $\left.(\mathrm{Hz})=\% 1.4 f^{\prime}, \mathrm{Fin}\right)$;

$\mathrm{s} 4=$ sprintf(' $\left.\quad \mathrm{BW}(\mathrm{Hz})=\% 1.0 f^{\prime}, \mathrm{bw}\right)$;

s5=sprintf(' OSR $\left.=\% 1.0 f^{\prime}, \mathrm{R}\right)$;

s6=sprintf(' Npoints $\left.=\% 1.0 f^{\prime}, \mathrm{N}\right)$;

s7=sprintf(' $\left.\operatorname{tsim}(\mathrm{sec})=\% 1.3 \mathrm{f}^{\prime}, \mathrm{N} / \mathrm{Fs}\right)$;

s8=sprintf(' Nperiods $\left.=\% 1.3 f^{\prime}, N^{\star} F i n / F s\right)$;

$\operatorname{disp}(\mathrm{s} 0)$

$\operatorname{disp}(\mathrm{s} 1)$

disp(s2)

$\operatorname{disp}(\mathrm{s} 3)$

$\operatorname{disp}(\mathrm{s} 4)$

$\operatorname{disp}(\mathrm{s} 5)$

$\operatorname{disp}(\mathrm{s} 6)$

$\operatorname{disp}(s 7)$

$\operatorname{disp}(\mathrm{s} 8)$

$\%$ \%

$\%$ Open Simulink diagram first 


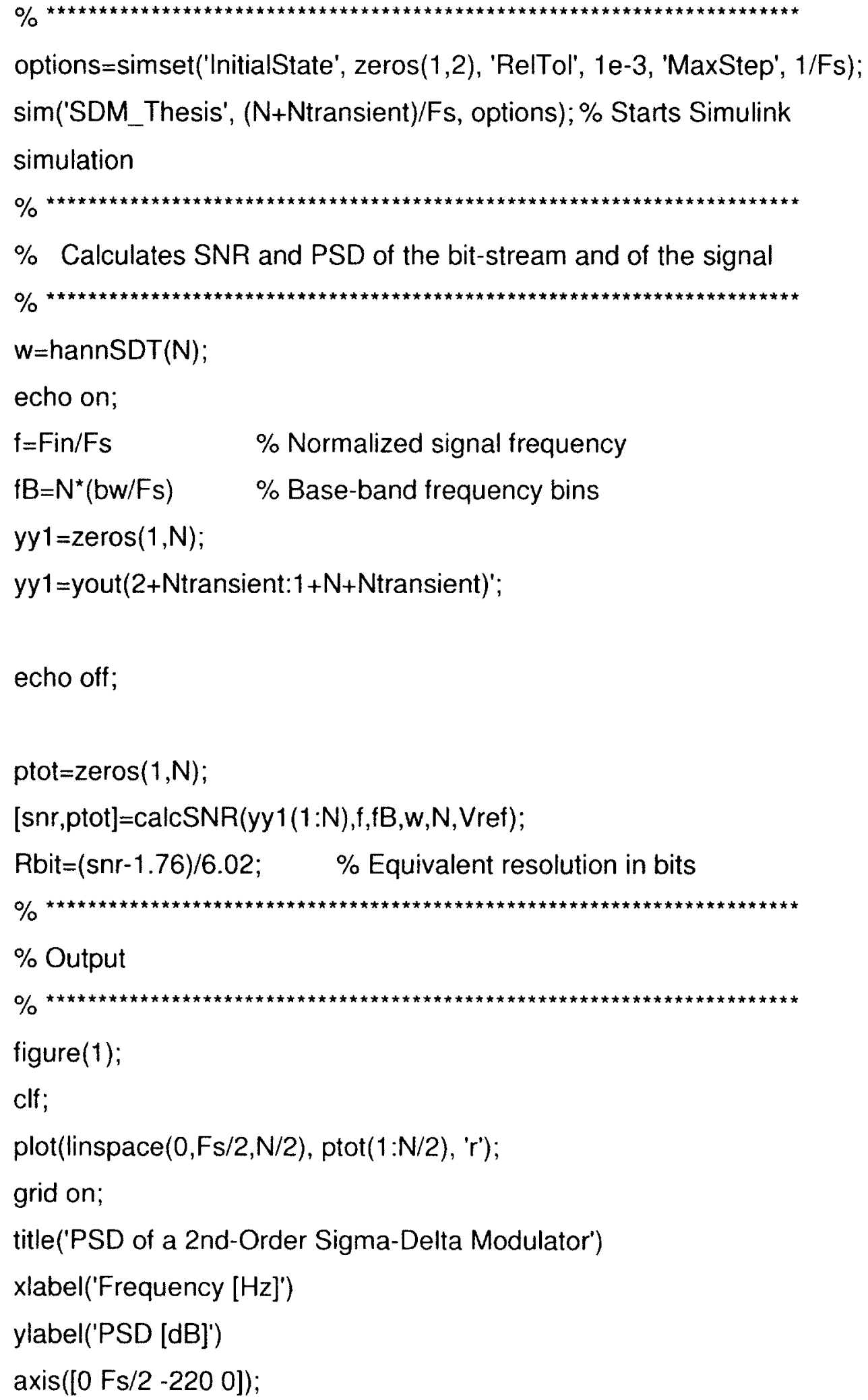


figure(2);

clf;

semilogx(linspace(0,Fs/2,N/2), ptot(1:N/2), 'r');

grid on;

title('PSD of a 2nd-Order Sigma-Delta Modulator')

xlabel('Frequency [Hz]')

ylabel('PSD [dB]')

axis([0 Fs/2 -220 0]);

figure(3);

clf;

plot(linspace $(0, F s / 2, N / 2)$, ptot(1:N/2), 'r');

hold on;

title('PSD of a 2nd-Order Sigma-Delta Modulator (detail)')

xlabel('Frequency [Hz]')

ylabel('PSD [dB]')

$\operatorname{axis}\left(\left[02^{*}(\mathrm{Fs} / \mathrm{R})-2200\right]\right)$;

grid on;

hold off;

text_handle = text(floor(Fs/R),-40, sprintf('SNR =\%4.1fdB @ OSR $=\% d \backslash n ', s n r, R)$ );

text_handle = text(floor(Fs/R),-60, sprintf('Rbit = \%2.2f bits @

OSR $=\% d \backslash n ', R b i t, R))$;

$\mathrm{s} 1=\operatorname{sprintf(}\left(\mathrm{SNR}(\mathrm{dB})=\% 1.3 \mathrm{f}^{\prime}, \mathrm{snr}\right)$;

$s 2=s p r i n t f(' \quad$ Simulation time $=\% 1.3 f$ min',etime $($ clock,t0)/60);

$\operatorname{disp}(\mathrm{s} 1)$

$\operatorname{disp}(\mathrm{s} 2)$

$\%$

$\%$ Histograms of the integrator outputs 


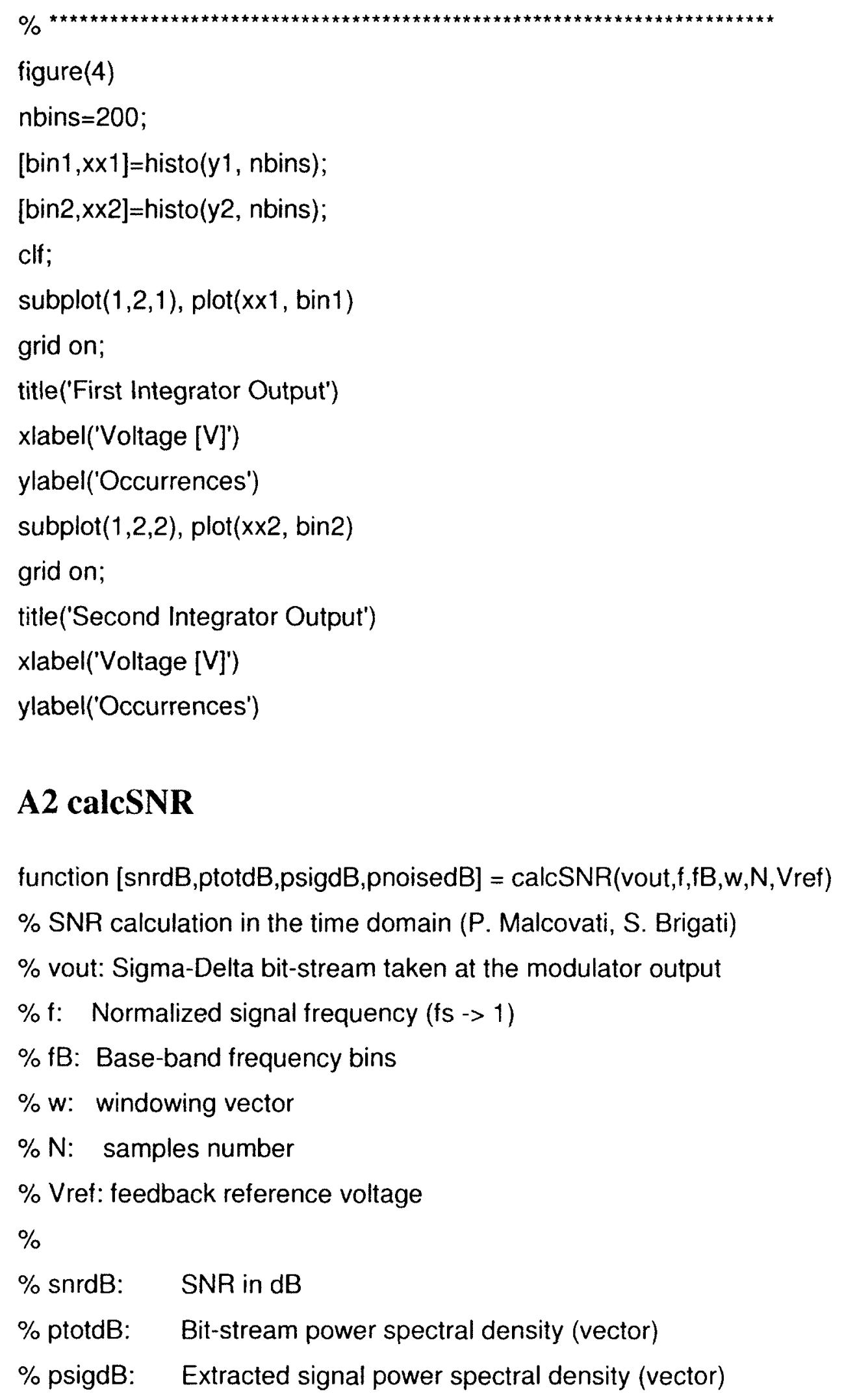

\section{A2 calcSNR}

function [snrdB,ptotdB,psigdB,pnoisedB] = calcSNR(vout,f,fB,w,N,Vref)

$\%$ SNR calculation in the time domain (P. Malcovati, S. Brigati)

$\%$ vout: Sigma-Delta bit-stream taken at the modulator output

$\% \mathrm{f}: \quad$ Normalized signal frequency (fs $->1$ )

$\% \mathrm{fB}$ : Base-band frequency bins

$\% \mathrm{w}$ : windowing vector

$\% \mathrm{~N}$ : samples number

$\%$ Vref: feedback reference voltage

$\%$

\% snrdB: $\quad$ SNR in $\mathrm{dB}$

$\%$ ptotdB: Bit-stream power spectral density (vector)

\% psigdB: Extracted signal power spectral density (vector) 


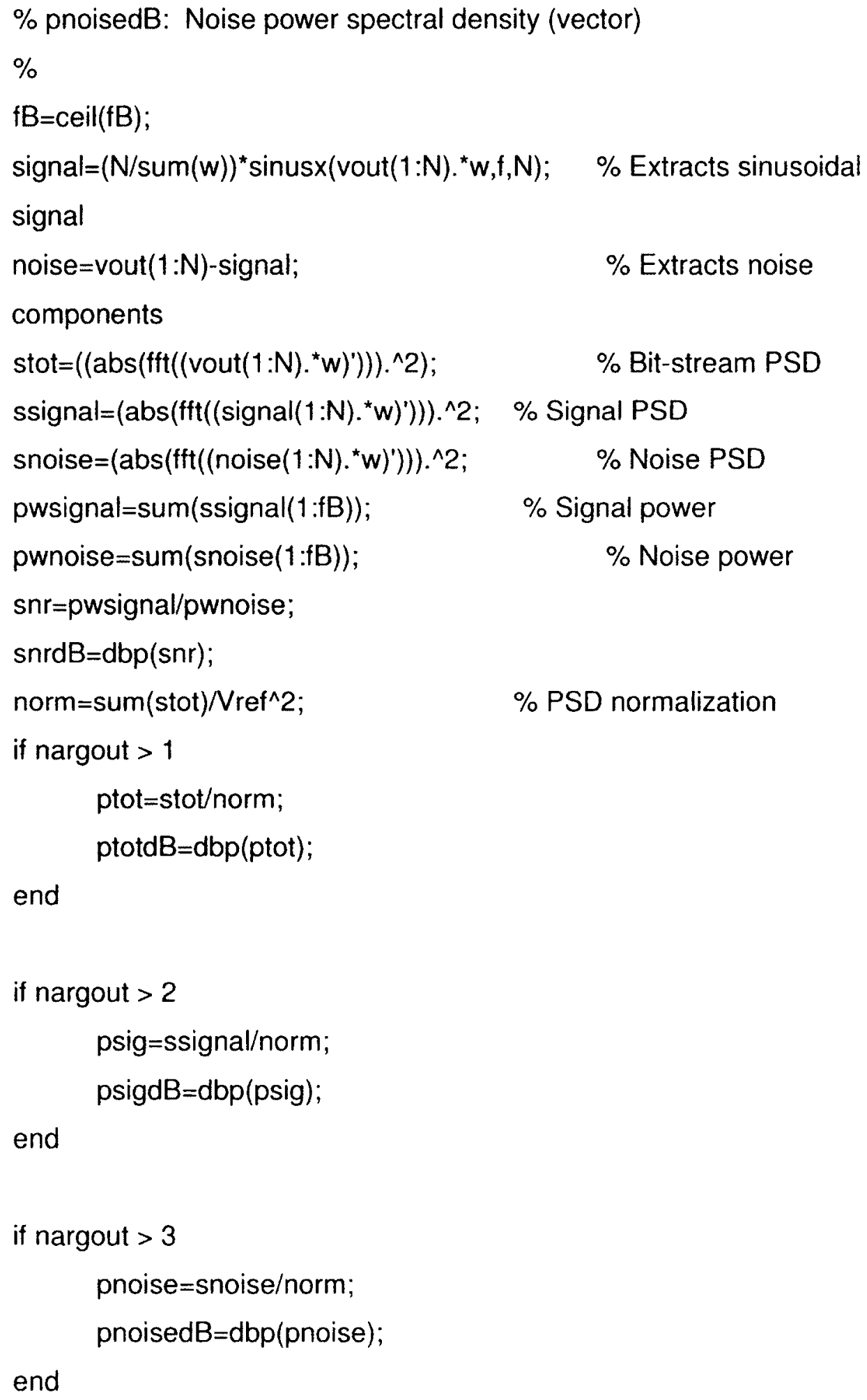




\section{A.3 sinusx}

function out $x=\sin u s x($ in $, f, n)$

$\%$ Extraction of a sinusoidal signal

$\%$

$\sin x=\sin \left(2^{*} \operatorname{pi}^{*} f^{*}[1: n]\right) ;$

$\cos x=\cos \left(2^{*} \mathrm{pi}^{*} \mathrm{f}^{*}[1: n]\right)$;

in $=$ in $(1: n)$;

a1 $=2^{*} \sin x .{ }^{*}$ in;

$a=\operatorname{sum}(a 1) / n$;

b1 $=2^{*} \cos x .{ }^{*}$ in;

$\mathrm{b}=\operatorname{sum}(\mathrm{b} 1) / \mathrm{n}$;

out $x=a .{ }^{*} \sin x+b .{ }^{*} \cos x$;

\section{A.4 hannSTD}

function $w=$ hannSDT $(n)$

$\%$ function $w=$ hannSDT $(n)$

$\%$ A Hann window of length $n$, does not smear tones located exactly in a bin.

$w=.5^{*}\left(1-\cos \left(2^{*} \mathrm{pi}^{*}(0: n-1) / \mathrm{n}\right)\right)$
A.5 histo
function $[\mathrm{bin}, \mathrm{xx}]=\operatorname{histo}(\mathrm{y}, \mathrm{N})$
$\%$ bins the elements of $Y$ into $N$ equally spaced containers within
$\%$ the maximum dynamic of $Y$.
$\%$
$\%$ bin: the number of occurrences for each bin
$\% \mathrm{xx}$ : is a vector returning the position of each bin 
range $=\operatorname{ceil}\left(2^{*} \max (y)^{\star} 10\right) / 10 ;$

$\mathrm{dx}=$ range $/ \mathrm{N}$;

for $i=1: N$

$$
x(i)=- \text { range } / 2+(i-1)^{\star} d x+d x / 2 ;
$$

end

$[$ bin, $x x]=h i s t(y, x)$;

\section{A.6 dbp}

function $\mathrm{y}=\mathrm{dbp}(\mathrm{x})$

$\% d b p(x)=10^{*} \log 10(x)$ : the $d B$ equivalent of the power $x$ $y=-\operatorname{lnf}^{*}$ ones $(\operatorname{size}(x))$;

nonzero $=x \sim=0$;

$y($ nonzero $)=10^{*} \log 10(\operatorname{abs}(x($ nonzero $)))$; 


\title{
Appendix B $\Delta \Sigma$ Modulator Source Code
}

\author{
B.1 Integrator \\ INerilog HDL for "VerilogD", "Integrator" "verilog" \\ module Integrator (Clk, Rst, Input, Output); \\ input Clk; \\ input Rst; \\ input [15:0] Input; \\ output [15:0] Output; \\ wire [15:0] Integ_in; \\ reg [15:0] Integ_input; \\ reg $[15: 0]$ Internal; \\ reg [15:0] Integ_output; \\ reg [15:0] Integ_outputP; \\ reg Sign; \\ reg [15:0] Diff; \\ assign Integ in = Input; \\ assign Output = Integ_output; \\ always @ (posedge Rst) \\ begin \\ Integ_input $<=16$ 'b 1000000000000000 \\ Integ_output $<=16$ 'b1000000000000000; \\ Integ_outputP $<=16$ 'b1000000000000000; \\ Internal $<=16$ 'b1000000000000000; \\ Sign $<=1$ 'b1; \\ end \\ always @(posedge Clk) \\ begin
}




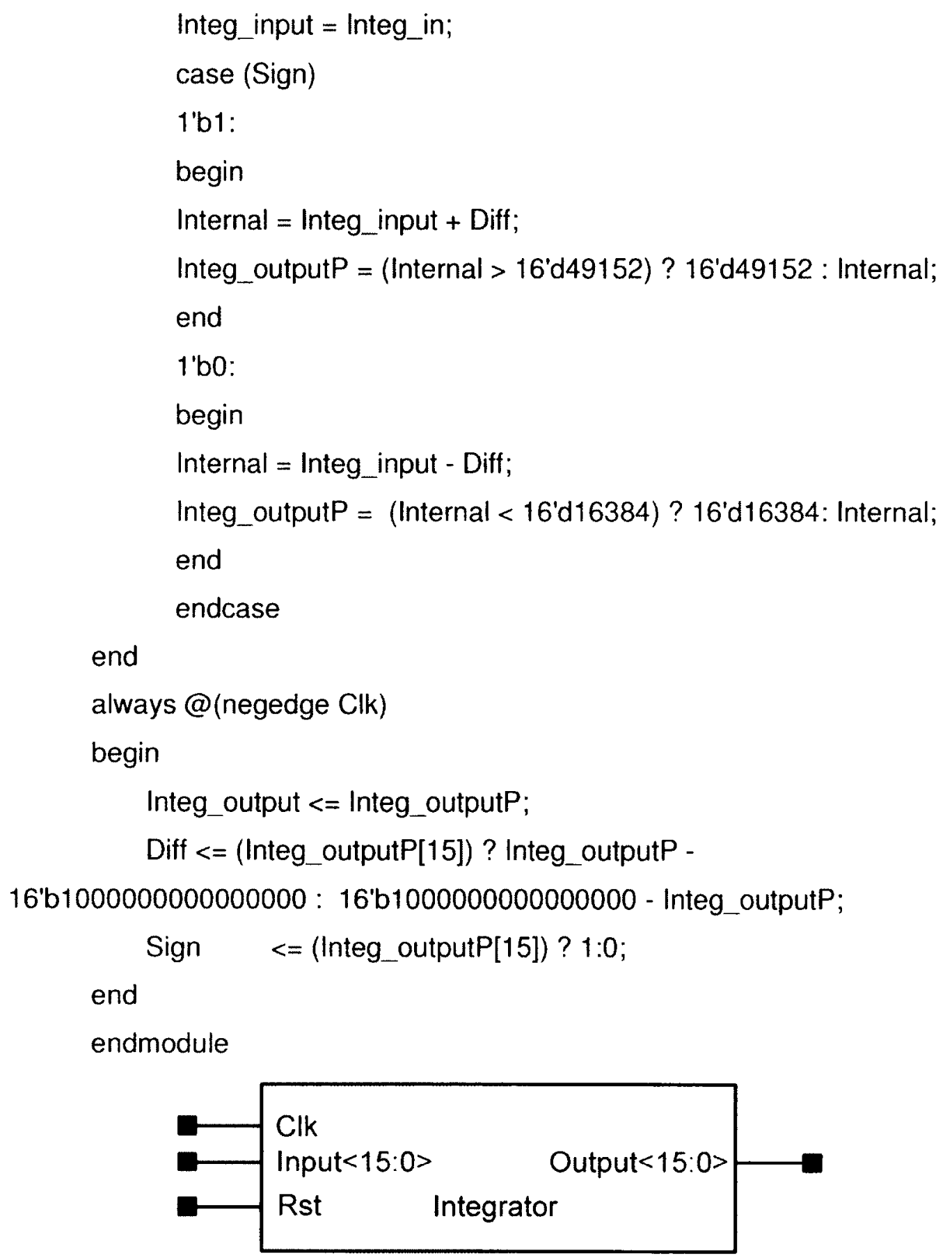

Figure B.1 Integrator Symbol 


\section{B.2 Adder 1}

INerilog HDL for "VerilogD", "Adder1" "verilog"

module Adder1 (Rst, In, Sign, Out);

input Rst;

input [15:0] In;

input Sign;

output [15:0] Output;

wire [15:0] InputW;

wire SignW;

reg [15:0] InputR;

reg SignR;

reg [15:0] OutputR;

assign InputW = In;

assign SignW = Sign;

assign Out = OutputR;

always @ (posedge Rst)

begin

InputR $<=16$ 'b1000000000000000;

SignR $<=1 \mathrm{~b} b 1$;

OutputR $<=16$ 'b1000000000000000;

end

always@ (InputW or SignW)

begin

InputR = InputW;

SignR = SignW;

case (SignR)

$1 ' b 1$ :

OutputR $=$ InputR -16 d $\mathrm{d} 5851 ; / /=16384^{*} 0.5 / 0.7=11702 / 2$ 
1 'bo:

OutputR $=$ Input $R+16^{\prime} d 5851 ; / /=16384^{*} 0.5 / 0.7=11702 / 2$

endcase

end

endmodule

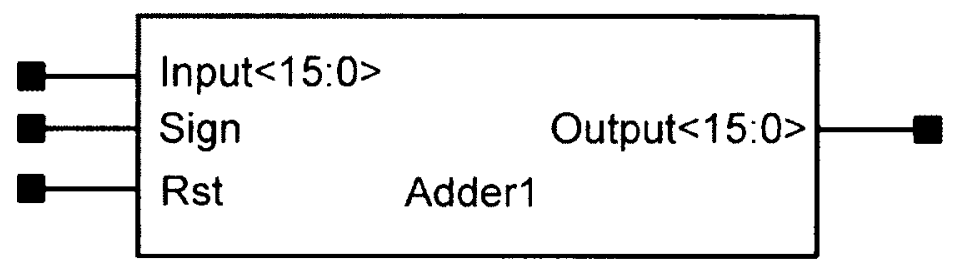

Figure B.2 Adder 1 Symbol

\section{B.3 Adder 2}

INerilog HDL for "VerilogD", "Adder2" "Пerilog"

module Adder2 (Rst, In, Sign, Out);

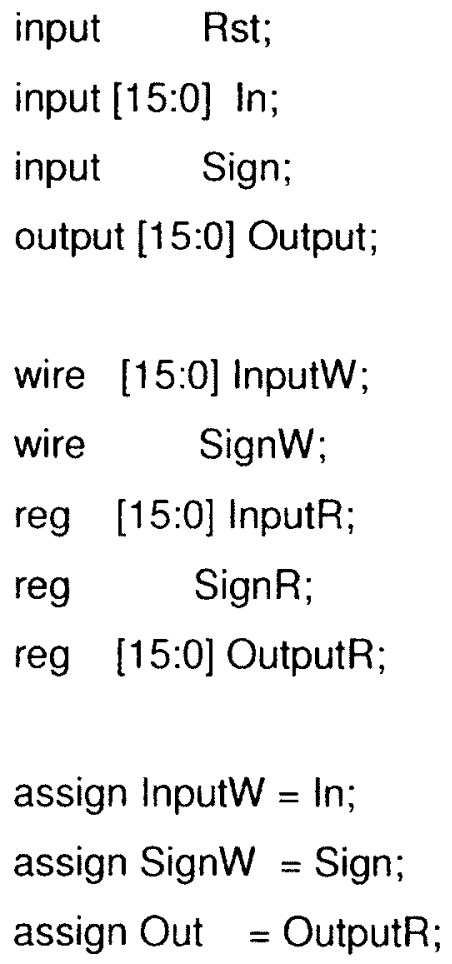


always @ (posedge Rst)

begin

InputR $<=16$ 'b1000000000000000;

SignR $<=1 ' b 1$;

OutputR $<=16$ 'b1000000000000000;

end

always @ (InputW or SignW)

begin

InputR = InputW;

SignR = SignW;

case (SignR)

1'b1:

OutputR $=\left(\right.$ InputR $-16^{\prime}$ d 11702) $>>1 ;=16384^{*} 0.5 / 0.7=11702$

1'b0:

Output $R=\left(\right.$ Input $\left.R+16^{\prime} d 11702\right)>>1 ;=16384^{*} 0.5 / 0.7=11702$ endcase

end

endmodule

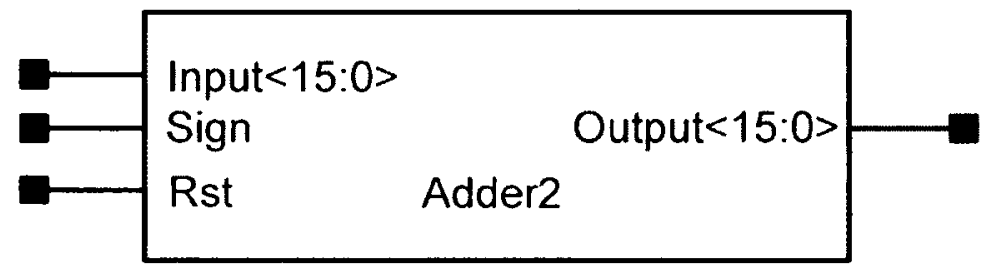

Figure A.3 Adder 2 Symbol

\section{B.4 Relay}

//Verilog HDL for "VerilogD", "Relay" "' ']erilog"

module Relay (Rst, In, Out); 


$$
\begin{aligned}
& \text { input Rst; } \\
& \text { input [15:0] In; } \\
& \text { output Out; } \\
& \text { wire [15:0] Input; } \\
& \text { reg Output; } \\
& \text { assign Input = In; } \\
& \text { assign Out = Output; }
\end{aligned}
$$
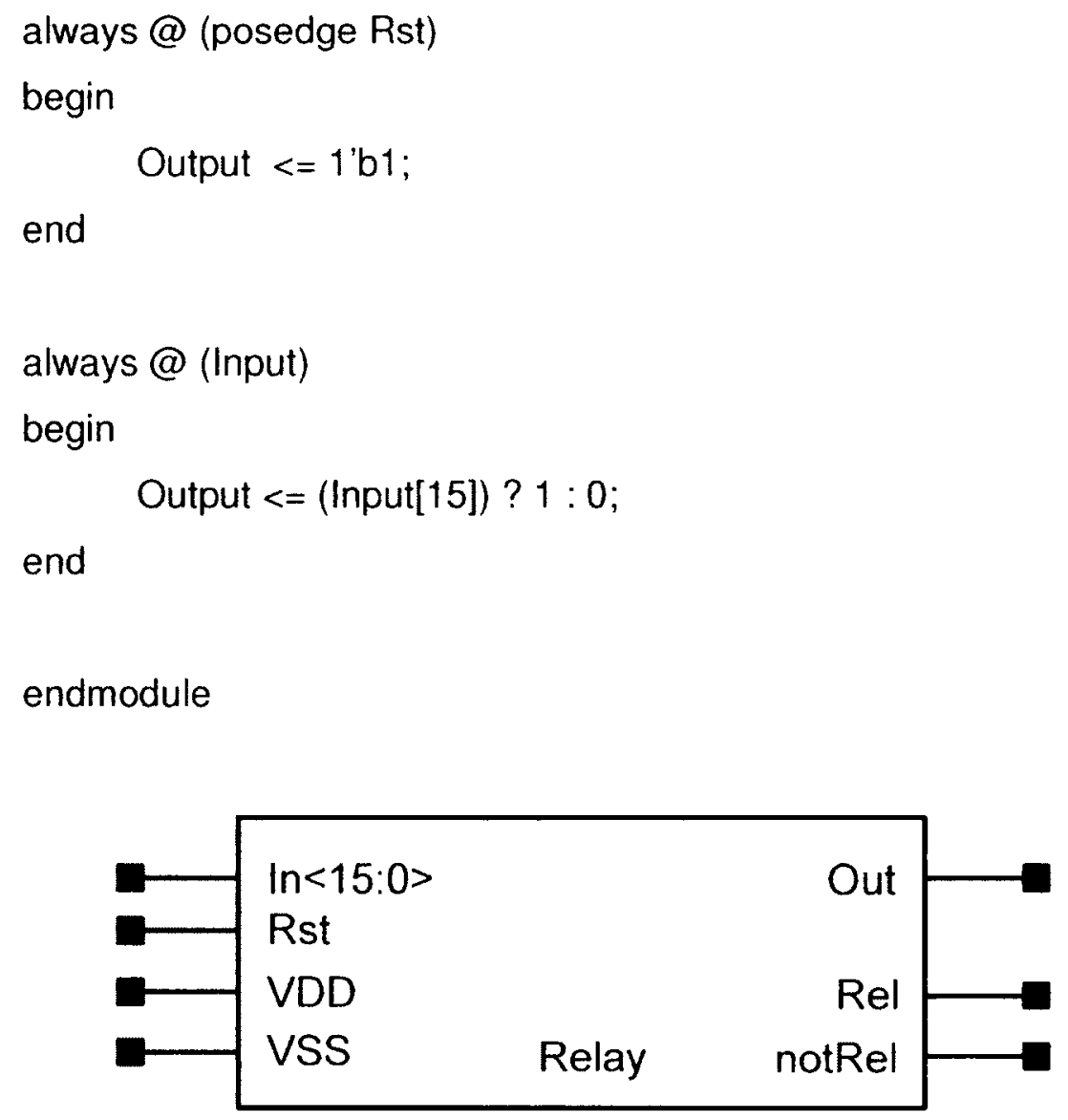

Figure B.4 Relay Symbol 


\section{Appendix C VerilogA Source Code}

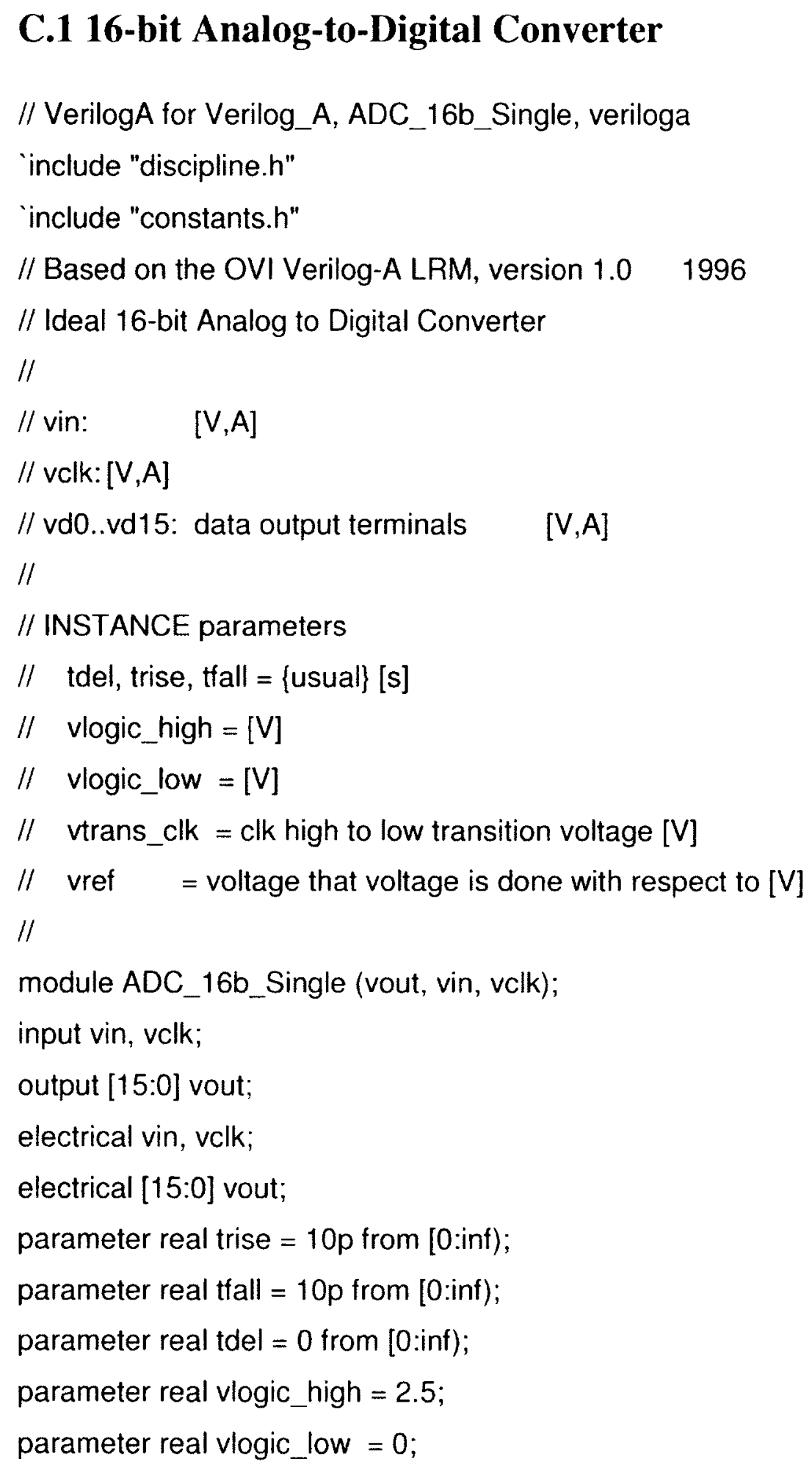




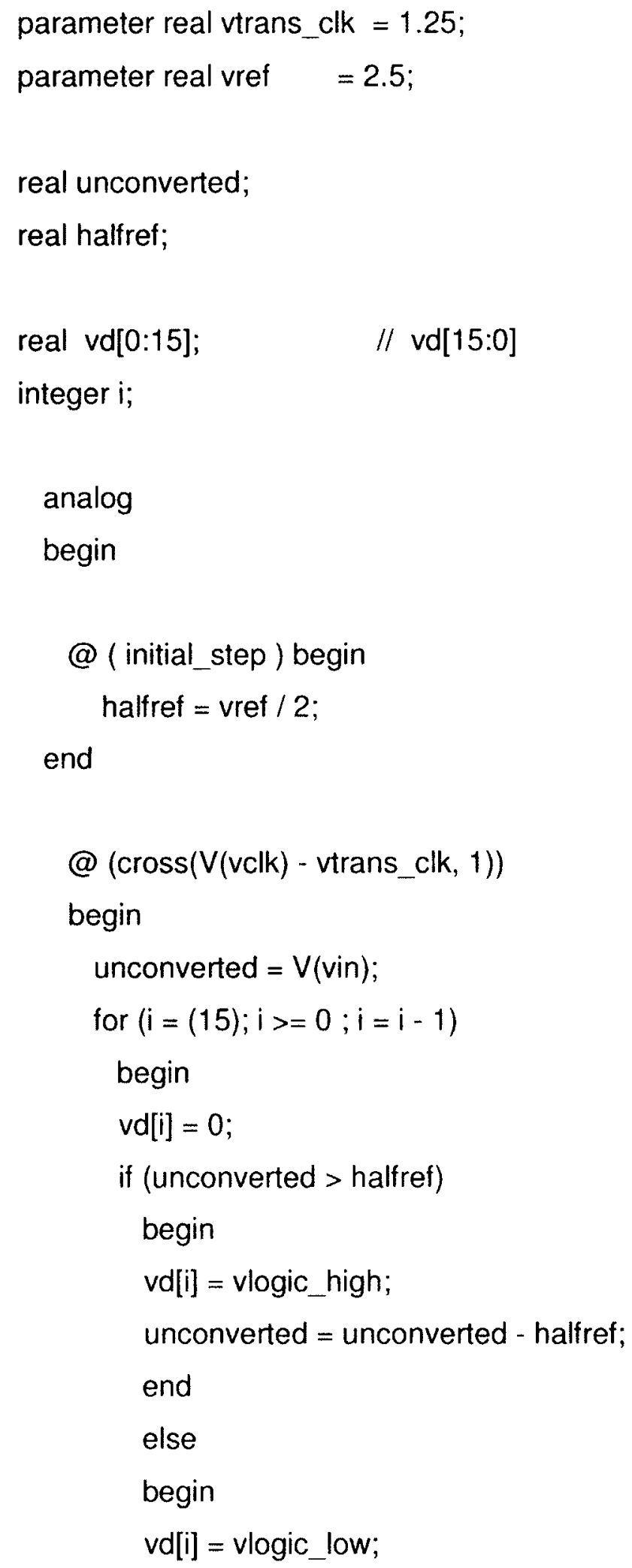




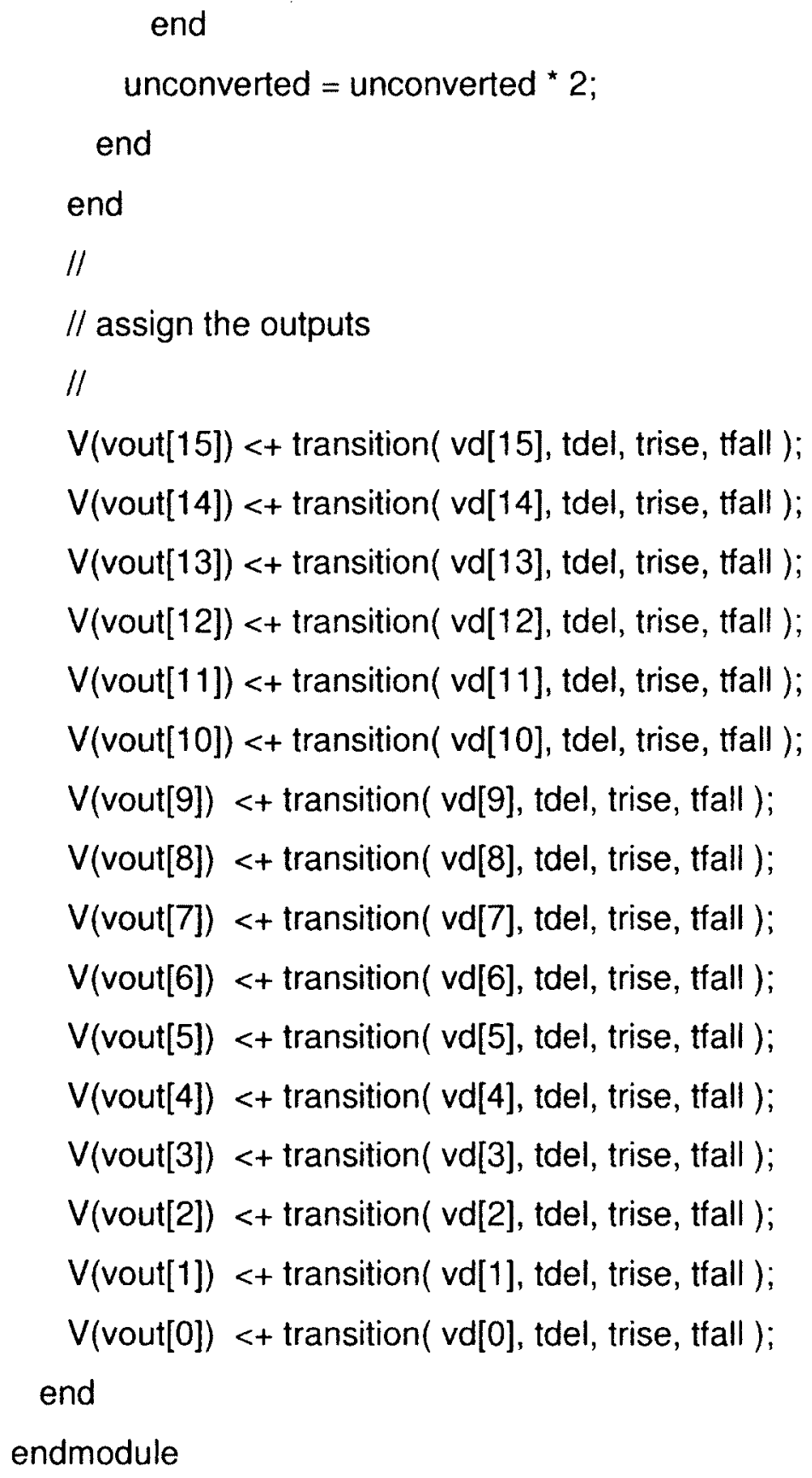




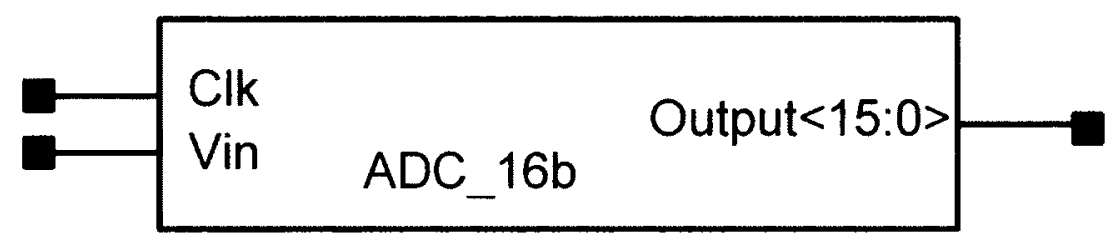

Figure C.1 16-bit ADC Symbol

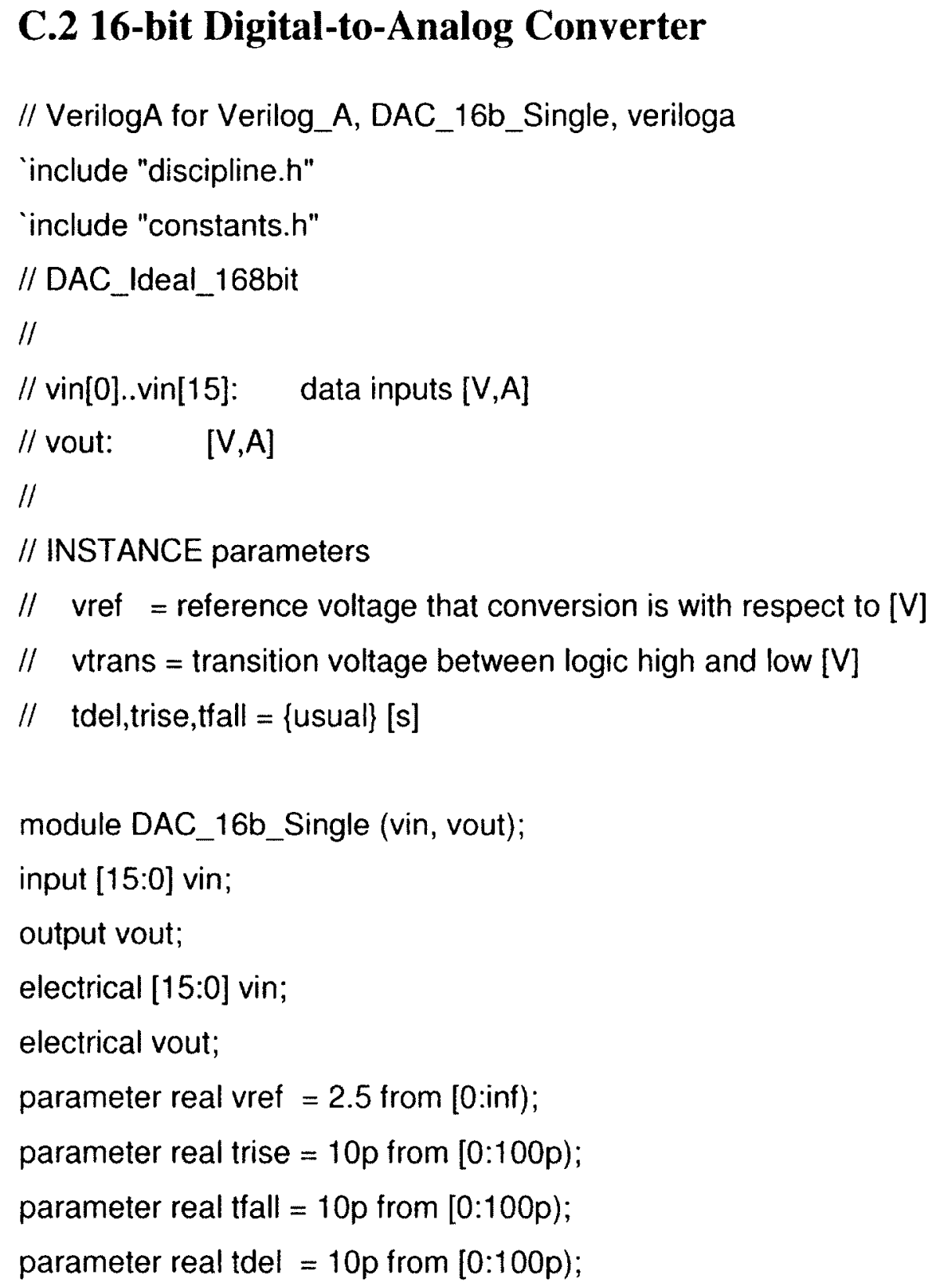


parameter real vtrans $=1.25$;

real out_scaled; // output scaled as fraction of 65536 analog begin

$$
\begin{aligned}
& \text { out_scaled }=0 \text {; } \\
& \text { out_scaled }=\text { out_scaled }+((\text { V }(\text { vin[15] })>\text { vtrans }) ? 32768: 0) \text {; } \\
& \text { out_scaled }=\text { out_scaled }+((\mathrm{V}(\operatorname{vin}[14])>\text { vtrans }) \text { ? } 16384: 0) \text {; } \\
& \text { out_scaled }=\text { out_scaled }+((\mathrm{V}(\mathrm{vin}[13])>\text { vtrans }) \text { ? } 8192: 0) \text {; } \\
& \text { out_scaled }=\text { out_scaled }+((\mathrm{V}(\mathrm{vin}[12])>\text { vtrans }) ? 4096: 0) \text {; } \\
& \text { out_scaled }=\text { out_scaled }+((\text { V }(\text { vin[11] })>\text { vtrans }) ? 2048: 0) \text {; } \\
& \text { out_scaled }=\text { out_scaled }+((\mathrm{V}(\mathrm{vin}[10])>\text { vtrans }) ? 1024: 0) \text {; } \\
& \text { out_scaled }=\text { out_scaled }+((\mathrm{V}(\mathrm{vin}[9])>\text { vtrans }) ? 512: 0) \text {; } \\
& \text { out_scaled }=\text { out_scaled }+((\mathrm{V}(\mathrm{vin}[8])>\text { vtrans }) ? 256: 0) \text {; } \\
& \text { out_scaled }=\text { out_scaled }+((\text { V }(\text { vin[7] })>\text { vtrans }) ? 128: 0) \text {; } \\
& \text { out_scaled }=\text { out_scaled }+((\text { V }(\text { vin }[6])>\text { vtrans }) ? 64: 0) \text {; } \\
& \text { out_scaled }=\text { out_scaled }+((V(\operatorname{vin}[5])>\text { vtrans }) ? 32: 0) \text {; } \\
& \text { out_scaled }=\text { out_scaled }+((\mathrm{V}(\operatorname{vin}[4])>\text { vtrans }) ? 16: 0) \text {; } \\
& \text { out_scaled }=\text { out_scaled }+((\text { V(vin[3]) }>\text { vtrans }) \text { ? } 8: 0) \text {; } \\
& \text { out_scaled }=\text { out_scaled }+((\text { V }(\text { vin[2] })>\text { vtrans }) ? 4: 0) \text {; } \\
& \text { out_scaled }=\text { out_scaled }+((\mathrm{V}(\mathrm{vin}[1])>\text { vtrans }) ? 2: 0) \text {; } \\
& \text { out_scaled }=\text { out_scaled }+((\mathrm{V}(\mathrm{vin}[0])>\text { vtrans }) \text { ? } 1: 0) \text {; } \\
& V(\text { vout) }<+ \text { transition( vref*out_scaled/65536, tdel, trise, tfall ); }
\end{aligned}
$$

end

endmodule

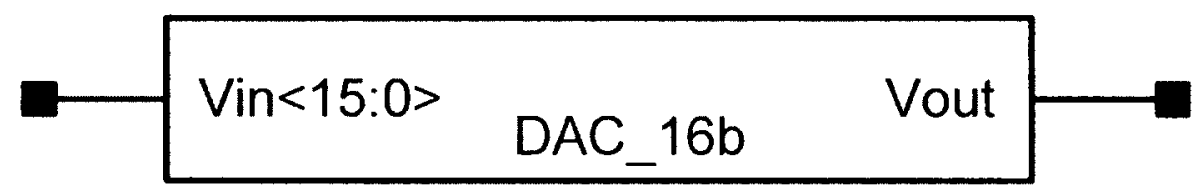

Figure C.2 16-bit DAC Symbol 